\title{
Dynamic of Bacterial and Phage Populations Maintained in Chemostat System with Dilution Control Parameter \\ Ofer Aluf
}

\author{
Netanya, Israel \\ oferaluf@bezeqint.net
}

\begin{abstract}
In this article, we discuss the crucial subject of bacteria and phage populations dynamic in chemostat system with dilution control parameter. A chemostat (from chemical environment is static) is a bioreactor to which fresh medium is continuously added, while culture liquid is continuously removed to keep the culture volume constant. By changing the rate with which medium is added to the bioreactor the growth rate of the microorganisms can be controlled. In chemostat model, the control parameters usually are the washout rate (dilution rate $D$ ) and input nutrient concentration $S^{(0)}$. We consider that $S^{(0)}$ is fixed and $D$ is the only control parameter. The outputs considered in chemostat models are functions: $\xi: R^{3} \rightarrow R^{k}(k \leq 3)$ of variables $S(t), V(t)$ and $B(t)$. The dynamical behavior of chemostat system with control tools is inspected and stability analysis is done. The chemostat system with dilution control parameter can be represented as differential equations, depending on variable and parameters. First the system is analyzed for zero talent period $(\tau=0)$ and secondly for positive value of talent period $(\tau>0)$.
\end{abstract}

Index Terms: Chemostat, Bioreactor, Dilution rate, Fixed point, Stability, Talent period, Delay Differential Equation (DDE).

\section{INTRODUCTION}

In this article, we discuss the crucial subject of bacteria and phage populations dynamic in chemostat system with dilution control parameter. In Chemostat model, the control variables employed are the washout rate (dilution rate $D$ ) and input nutrient concentration $S^{(0)}$. First we consider that $S^{(0)}$ is fixed and $D$ will be the only control variable. The outputs usually considered in Chemostat models are functions: $\xi: R^{3} \rightarrow R^{k} R^{3} \rightarrow R^{k}(k \leq 3)$ of variables $S(t), V(t)$ and $B(t)$. We consider that dilution rate $D$ is the feedback control variable of the system and define the feedback control law $D: R_{+}^{3} \rightarrow R_{+}$by $D(B, V, S)=\xi(B, V, S)$ where $\xi: R_{+} \rightarrow R_{+}$is a continuously differentiable. $D[1 / \mathrm{sec}]$, $B[1 / m l], V[1 / m l], S[1 / m l]$. We define washout rate balance parameters $\mathrm{k}_{i}[\mathrm{ml} / \mathrm{sec}]$.Dilution rate function: $D=\xi(B, V, S)$ $D=\xi(B, V, S)=B k_{1}+V k_{2}+S k_{3}$ [1] [2] [3].

*Corresponding author. Tel.: +972 98320206 E-mail address: oferaluf@bezeqint.net (O. Aluf).

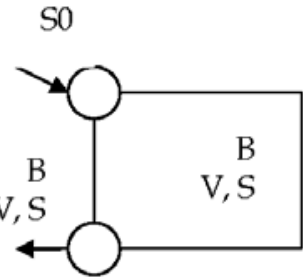

$$
D=\xi(B, V, S)=B k_{1}+V k_{2}+S k_{3}
$$

Fig. 1. Chemostat system with dilution control parameter.

$$
\begin{aligned}
& D\left[\frac{1}{\mathrm{sec}}\right]=B\left[\frac{1}{\mathrm{ml}}\right] k_{1}\left[\frac{\mathrm{ml}}{\mathrm{gec}}\right]+V\left[\frac{1}{\mathrm{ml}}\right] k_{2}\left[\frac{\mathrm{ml}}{\mathrm{sec}}\right] \\
& +S\left[\frac{1}{\mathrm{ml}}\right] k_{3}\left[\frac{\mathrm{ml}}{\mathrm{gec}}\right]
\end{aligned}
$$

Terminology:

$V$ - Phage concentration in the vessel.

$B$ - Bacteria concentration in the vessel.

$S^{(0)}$ - Input concentration of the limiting nutrient

$S$ - Current concentration of the nutrient in the vessel.

System chemostat (1compartment gradostat) equations:

$$
\begin{gathered}
\frac{d V(t)}{d t}=-V(t)-\gamma V(t) B(t) \\
+\beta \gamma V(t-\tau) B(t-\tau)-V(t) D \\
\frac{d B(t)}{d t}=\psi f(S) B(t)-\gamma V(t) B(t)-B(t) D \\
\frac{d S(t)}{d t}=\left[S^{(0)}-S(t)\right] D-\psi f(S) B(t) / \delta \\
D=\xi(B, V, S)=B k_{1}+V k_{2}+S k_{3}
\end{gathered}
$$

$\varphi$ - Decay rate of phage in the vessel.

$\beta$ - Average number of free phage released at lysing in the vessel

$\psi$ - Growth rate of bacteria in the vessel.

$\gamma$ - Interaction rate between bacteria and phage in the vessel. $\tau$ - Talent period. $D$ - Washout rate.

$S^{(0)}$ - Input concentration of the limiting nutrient.

$\delta$ - Conversion factor in the vessel.

$k_{1}, k_{2}, k_{3}$ - Washout rate balance parameters. 
$S^{(0)}>=0, \quad B(0)>=0, \quad V(0)>=0$. Chemostat washout term $\left[S^{(0)}-S(t)\right] D$. For the case of simplicity we assume no delay $\tau=0$.

$$
\begin{aligned}
& \frac{d V(t)}{d t}=-\varphi V(t)+\gamma V(t) B(t)(\beta-1) \\
& -V(t)\left[B(t) k_{1}+V(t) k_{2}+S(t) k_{3}\right] \\
& \frac{d B(t)}{d t}=\psi f(S) B(t)-\gamma V(t) B(t) \\
& -B(t)\left[B(t) k_{1}+V(t) k_{2}+S(t) k_{3}\right] \\
& \frac{d S(t)}{d t}=\left[S^{(0)}-S(t)\right]\left[B(t) k_{1}+V(t) k_{2}+S(t) k_{3}\right]
\end{aligned}
$$$$
-\psi f(S) B(t) / \delta
$$

$$
V=V(t) ; B=B(t) ; S=S(t)
$$

$\frac{d V}{d t}=-\varphi V+\gamma V B(\beta-1)-V\left[B k_{1}+V k_{2}+S k_{3}\right]$

$\frac{d B}{d t}=\psi f(S) B-\gamma V B-B\left[B k_{1}+V k_{2}+S k_{3}\right]$

$\frac{d S}{d t}=\left[S^{(0)}-S\right]\left[B k_{1}+V k_{2}+S k_{3}\right]-\psi f(S) B / \delta$

Scaling of the above equation: All resources densities in term of $S^{(0)}$ and get

$$
\frac{S}{S^{(0)}} \rightarrow S ; \frac{V}{S^{(0)} \beta \delta} \rightarrow V ; \frac{B}{S^{(0)} \delta} \rightarrow B ; a \rightarrow \frac{a}{S^{(0)}}
$$

$t \rightarrow t D$. Dimensional parameters have the following meaning:

$$
\begin{aligned}
& \psi \rightarrow \frac{\psi}{D} ; \gamma \rightarrow \frac{\gamma \beta S^{(0)} \delta}{D} ; k_{1} \rightarrow k_{1} \delta S^{(0)} \\
& k_{2} \rightarrow k_{2} S^{(0)} \beta \delta ; k_{3} \rightarrow S^{(0)} k_{3} ; S^{(0)} \geq 0 \\
& B(0)>0 ; \frac{S}{S^{(0)}} \rightarrow S \\
& \frac{a}{S^{(0)}} \rightarrow a ; f(S)=\frac{V_{\max } S / S^{(0)}}{a / S^{(0)}+S / S^{(0)}}
\end{aligned}
$$

Input concentration of the limiting nutrient $S^{(0)}$ term has units of concentration. $D[1 / \mathrm{sec}]$ has units of reciprocal time. Let scale the nutrient equation:

$$
\frac{d S}{d t}=\left[S^{(0)}-S\right]\left[B k_{1}+V k_{2}+S k_{3}\right]-\psi f(S) B / \delta
$$

Divide the two equation sides by $S^{(0)}$ term:

$$
\frac{d S}{d t} \frac{1}{S(0)}=\left[1-\frac{S}{S(0)}\right]\left[\frac{B}{S^{(0)} \delta} k_{1} \delta S^{(0)}+\frac{V}{S^{(0)} \beta \delta} k_{2} S^{(0)} \beta \delta\right.
$$$$
\left.+\frac{S}{S(0)} S^{(0)} k_{3}\right]-\psi f(S) B /\left[S^{(0)} \delta\right]
$$

Measuring $S, a, B / \delta$ in units of $\mathrm{S}^{(0)}$, and time in units of $D^{-1}$

$$
\begin{aligned}
& \frac{B\left[\frac{1}{m l}\right]}{S^{(0)}\left[\frac{1}{m l}\right]} k_{1}\left[\frac{m l}{\mathrm{sec}}\right] \delta S^{(0)}\left[\frac{1}{m l}\right] \rightarrow\left[\frac{1}{\mathrm{sec}}\right] \\
& \frac{V\left[\frac{1}{m l}\right]}{S^{(0)}\left[\frac{1}{m l}\right] \beta \delta} k_{2}\left[\frac{m l}{\mathrm{sec}}\right] S^{(0)}\left[\frac{1}{m l}\right] \beta \delta \rightarrow\left[\frac{1}{\mathrm{sec}}\right] \\
& \frac{S\left[\frac{1}{m l}\right]}{S^{(0)}\left[\frac{1}{m l}\right]} S^{(0)}\left[\frac{1}{m l}\right] k_{3}\left[\frac{m l}{\mathrm{sec}}\right] \rightarrow\left[\frac{1}{\mathrm{sec}}\right]
\end{aligned}
$$

The scales nutrient equation is:

$$
\frac{d S}{d t}=(1-S)\left(B k_{1}+V k_{2}+S k_{3}\right)-\psi f(S) B
$$

Let take the phages differential equation:

$$
\begin{gathered}
\frac{V}{S^{(0)} \beta \delta} \rightarrow V ; \frac{\varphi\left[\frac{1}{\mathrm{sec}}\right]}{D\left[\frac{1}{\mathrm{sec}}\right]} \rightarrow \varphi \\
\frac{d V}{d t}=-\varphi V+\gamma V B(\beta-1)-V\left[B k_{1}+V k_{2}+S k_{3}\right]
\end{gathered}
$$

Rewriting $V(t)$ phage density in terms of $S^{(0)} \beta \delta$

$$
\begin{aligned}
& \frac{d V}{d t} \frac{1}{S^{(0)} \beta \delta}=-\varphi V \frac{1}{S^{(0)} \beta \delta}+\gamma V \frac{1}{S^{(0)} \beta \delta} B(\beta-1) \\
& -V \frac{1}{S(0) \beta \delta}\left[\frac{B}{S^{(0)} \delta} k_{1} S^{(0)} \delta+V \frac{1}{S^{(0)} \beta \delta} k_{2} S^{(0)} \beta \delta\right. \\
& \left.+\frac{S}{S^{(0)}} k_{3} S^{(0)}\right] \\
& \quad \frac{d V}{d t} \frac{1}{S^{(0)} \beta \delta}=-\varphi V \frac{1}{S^{(0)} \beta \delta} \\
& \quad+\gamma \frac{S^{(0)} \beta \delta}{D} V \frac{1}{D} B \frac{D}{S^{(0)} \beta \delta}(\beta-1) \\
& \quad-V \frac{1}{S^{(0)} \beta \delta}\left[\frac{B}{S^{(0)} \delta} k_{1} S^{(0)} \delta\right. \\
& \left.+V \frac{1}{S^{(0)} \beta \delta} k_{2} S^{(0)} \beta \delta+\frac{S}{S^{(0)}} k_{3} S^{(0)}\right]
\end{aligned}
$$

$$
\frac{d V}{d t} \frac{1}{S^{(0)} \beta \delta}=-\varphi V \frac{1}{S^{(0)} \beta \delta}
$$

$$
+\gamma \frac{S^{(0)} \beta \delta}{D} V \frac{1}{S^{(0)} \beta \delta} B \frac{1}{S^{(0)} \delta} \frac{D}{\beta}(\beta-1)
$$$$
-V \frac{1}{S^{(0)} \beta \delta}\left[\frac{B}{S^{(0)} \delta} k_{1} S^{(0)} \delta+V \frac{1}{S^{(0)} \beta \delta} k_{2} S^{(0)} \beta \delta\right.
$$$$
\left.+\frac{S}{S(0)} k_{3} S^{(0)}\right]
$$

$$
V \frac{1}{S^{(0)} \beta \delta} \rightarrow V ; B \frac{1}{S^{(0)} \delta} \rightarrow B ; \gamma \frac{S^{(0)} \beta \delta}{D} \rightarrow \gamma
$$

$$
\begin{gathered}
\varphi \rightarrow \frac{\varphi}{D} ; \psi \rightarrow \frac{\psi}{D} ; \gamma \rightarrow \frac{\gamma \beta S^{(0)} \delta}{D} ; k_{1} \rightarrow k_{1} \delta S^{(0)} \\
k_{2} \rightarrow k_{2} S^{(0)} \beta \delta ; k_{3} \rightarrow S^{(0)} k_{3} \\
S^{(0)} \geq 0 ; B(0)>0 ; \frac{S}{S^{(0)}} \rightarrow S \\
\frac{a}{S^{(0)}} \rightarrow a ; f(S)=\frac{V_{\max } S / S^{(0)}}{a / S^{(0)}+S / S^{(0)}} \\
\frac{S}{S^{(0)}} \rightarrow S ; \frac{V}{S^{(0)} \beta \delta} \rightarrow V ; \frac{B}{S^{(0)} \delta} \rightarrow B \\
a \rightarrow \frac{a}{S(0)} ; t \rightarrow t D
\end{gathered}
$$

$\frac{d V}{d t}=-\varphi V+\gamma V B \frac{D}{\beta}(\beta-1)-V\left[B k_{1}+V k_{2}+S k_{3}\right](27)$ Measuring $S, a, B / \delta_{1}$ in units of $S^{(0)}$, and time in units of $D^{-1}$

$$
\begin{aligned}
& \frac{d V}{d t} \frac{1}{D}=-\frac{\varphi}{D} V+\gamma V B \frac{D}{\beta} \frac{1}{D}(\beta-1) \\
& -V\left[B k_{1}+V k_{2}+S k_{3}\right] \frac{1}{D}
\end{aligned}
$$

$$
\frac{d V}{d t}=-\varphi V+\gamma V B \frac{(\beta-1)}{\beta}-V\left[B k_{1}+V k_{2}+S k_{3}\right]
$$

$$
\frac{d V}{d t}=-\varphi V+\gamma V B\left(1-\frac{1}{\beta}\right)-V\left[B k_{1}+V k_{2}+S k_{3}\right]
$$

Now let scale the Bacteria equations:

$$
\frac{d B}{d t}=\psi f(S) B-\gamma V B-B\left[B k_{1}+V k_{2}+S k_{3}\right]
$$

We write the Bacteria densities in term of $S^{(0)} \delta$ 


$$
\begin{aligned}
\frac{B}{S(0) \delta} & \rightarrow B ; \frac{V}{S(0) \beta \delta} \rightarrow V ; \gamma \rightarrow \frac{\gamma \beta S^{(0)} \delta}{D} \\
f(S) & =\frac{V_{\max } S / S^{(0)}}{a / S^{(0)}+S / S^{(0)}}
\end{aligned}
$$

Multiply two equation sides by factor $\frac{1}{S^{(0)} \delta}$

$$
\begin{gathered}
\quad \frac{d B}{d t} \frac{1}{S^{(0)} \delta}=\psi f(S) B \frac{1}{S^{(0)} \delta}-\gamma V B \frac{1}{S^{(0)} \delta} \\
-B \frac{1}{S^{(0)} \delta}\left[B k_{1}+V k_{2}+S k_{3}\right] \\
\frac{d B}{d t} \frac{1}{S^{(0)} \delta}=\psi f(S) B \frac{1}{S^{(0)} \delta}-\gamma V B \frac{1}{S^{(0)} \delta} \\
-B \frac{1}{S^{(0)} \delta}\left[\frac{B}{S^{(0)} \delta} k_{1} S^{(0)} \delta+V \frac{1}{S^{(0)} \beta \delta} k_{2} S^{(0)} \beta \delta\right. \\
\left.+\frac{S}{S^{(0)}} k_{3} S^{(0)}\right] \\
\frac{d B}{d t}=\psi f(S) B-\gamma V D B-B\left[B k_{1}+V k_{2}+S k_{3}\right] \\
\frac{d B}{d t} \frac{1}{D}=\psi \frac{1}{D} f(S) B-\gamma V B-B\left[B k_{1}+V k_{2}+S k_{3}\right] \frac{1}{D} \\
\frac{d B}{d t} \frac{1}{D} \rightarrow \frac{d B}{d t} ; \psi \frac{1}{D} \rightarrow \psi \\
{\left[B k_{1}+V k_{2}+S k_{3}\right] \frac{1}{D} \rightarrow\left[B k_{1}+V k_{2}+S k_{3}\right]} \\
\frac{d B}{d t}=\psi f(S) B-\gamma V B-B\left[B k_{1}+V k_{2}+S k_{3}\right]
\end{gathered}
$$

The Chemostat (One compartment Gradostat) scaled equations with control parameters are

$$
\begin{gathered}
\frac{d B}{d t}=\psi f(S) B-\gamma V B-B\left[B k_{1}+V k_{2}+S k_{3}\right] \\
\frac{d V}{d t}=-\varphi V+\gamma V B\left(1-\frac{1}{\beta}\right)-V\left[B k_{1}+V k_{2}+S k_{3}\right] \\
\frac{d S}{d t}=(1-S)\left(B k_{1}+V k_{2}+S k_{3}\right)-\psi f(S) B
\end{gathered}
$$

Remark: In the chemostat parameters scaling process we get un-dimensional parameters. If the system is a fixed point there is no nutrient, phage and bacteria change in time $\frac{d S}{d t}=0 ; \frac{d V}{d t}=0 ; \frac{d B}{d t}=0$. We get three algebraic equations which give the expression for those three fixed point values. Our Chemostat system dynamic develops in time to bring us to fixed points. We sign the maximal rate bacteria reaction to nutrient as $V_{M}, V_{m}$, or $V_{\max }$ in our analysis. We define: $B(t)=B ; S(t)=S ; V(t)=V$

$$
\begin{gathered}
f_{1}(S, V, B)=(1-S)\left(B k_{1}+V k_{2}+S k_{3}\right)-\psi f(S) B \\
f_{2}(S, V, B)=-\varphi V+\gamma V B\left(1-\frac{1}{\beta}\right) \\
-V\left[B k_{1}+V k_{2}+S k_{3}\right]
\end{gathered}
$$

$f_{3}(S, V, B)=\psi f(S) B-\gamma V B-B\left[B k_{1}+V k_{2}+S k_{3}\right]$

To find the fixed points for the system, we solve $\frac{d S}{d t}=0 ; \frac{d V}{d t}=0 ; \frac{d B}{d t}=0$ i.e.:

$$
(1-S)\left(B k_{1}+V k_{2}+S k_{3}\right)-\psi f(S) B=0
$$

$$
-\varphi V+\gamma V B\left(1-\frac{1}{\beta}\right)-V\left[B k_{1}+V k_{2}+S k_{3}\right]=0
$$

$$
\psi f(S) B-\gamma V B-B\left[B k_{1}+V k_{2}+S k_{3}\right]=0
$$

From the second equation $f_{2}(S, V, B)$ :

$$
\begin{aligned}
& V\left\{-\varphi+\gamma B\left(1-\frac{1}{\beta}\right)-\left[B k_{1}+V k_{2}+S k_{3}\right]\right\}=0 \\
& V=0 \text { or }-\varphi+\gamma B\left(1-\frac{1}{\beta}\right)-\left[B k_{1}+V k_{2}+S k_{3}\right]=0
\end{aligned}
$$

Case No.1: $V=0$ : Third equation gives:

$$
\begin{aligned}
& \psi f(S) B-\gamma V B-B\left[B k_{1}+V k_{2}+S k_{3}\right] \\
& \psi f(S) B-B\left[B k_{1}+S k_{3}\right] \\
& \quad \psi f(S) B-B\left[B k_{1}+S k_{3}\right] \\
& \quad \Rightarrow B\left\{\psi f(S)-\left[B k_{1}+S k_{3}\right]\right\}=0
\end{aligned}
$$

Case No.1.1: Choosing $B=0$ give from first equation

$$
\begin{aligned}
& (1-S)\left(B k_{1}+V k_{2}+S k_{3}\right)-\psi f(S) B \\
& (1-S)\left(S k_{3}\right)=0 \Rightarrow S=0 \text { or } \mathrm{S}=1
\end{aligned}
$$

The first fixed point: $E^{(0)}\left(V^{(0)}, B^{(0)}, S^{(0)}\right)=(0,0,0)$

The second fixed point: $E^{(1)}\left(V^{(1)}, B^{(1)}, S^{(1)}\right)=(0,0,1)$

Case No.1.2: Choosing

$$
\psi f(S)-\left[B k_{1}+S k_{3}\right]=0 \Rightarrow B=\frac{\psi f(S)-S k_{3}}{k_{1}}
$$

It gives from first equation

$$
\begin{gathered}
(1-S)\left(B k_{1}+V k_{2}+S k_{3}\right)-\psi f(S) B \Rightarrow V=0 \\
(1-S)\left(\left[\frac{\psi f(S)-S k_{3}}{k_{1}}\right] k_{1}+S k_{3}\right) \\
-\psi f(S)\left(\frac{\psi f(S)-S k_{3}}{k_{1}}\right)=0 \\
(1-S)\left(\psi f(S)-S k_{3}+S k_{3}\right) \\
-\psi f(S)\left(\frac{\psi f(S)-S k_{3}}{k_{1}}\right)=0 \\
(1-S)[\psi f(S)]-\psi f(S)\left[\frac{\psi f(S)-S k_{3}}{k_{1}}\right]=0 \\
\psi f(S)\left\{(1-S)-\left[\frac{\psi f(S)-S k_{3}}{k_{1}}\right]\right\}=0 \\
f(S)=0 \text { or }(1-S)-\left[\frac{\psi f(S)-S k_{3}}{k_{1}}\right]=0 \\
f(S)=0 \Rightarrow \frac{V_{\max } S}{a+S} \Rightarrow S=0 \\
\left.B\right|_{S=0}=\frac{\psi f(S=0)}{k_{1}}=0 ; f(S)=\frac{V_{\max } S}{a+S} \\
(1-S)-\left[\frac{\psi f(S)-S k_{3}}{k_{1}}\right]=0 \\
1-S-\frac{\psi}{k_{1}} f(S)+\frac{S k_{3}}{k_{1}}=0
\end{gathered}
$$


Ofer Aluf; Dynamic of Bacterial and Phage Populations Maintained in Chemostat System with Dilution Control Parameter, Transactions on Networks and Communications, Volume 4 No. 3, June (2016); pp: 1-21

$$
\begin{aligned}
& 1-S-\frac{\psi}{k_{1}} f(S)+\frac{S k_{3}}{k_{1}}=0 \\
& 1-S-\frac{\psi}{k_{1}}\left[\frac{V_{\text {max }} S}{a+S}\right]+\frac{k_{k}}{k_{1}}=0 \\
& 1+S\left[\frac{k_{3}}{k_{1}}-1\right]-\frac{\psi}{k_{1}}\left[\frac{V_{\max } S}{a+S}\right]=0 \\
& a+S+S\left[\frac{k_{2}}{k_{1}}-1\right](a+S)-\frac{\psi}{k_{1}} V_{\max } S=0 \\
& S^{2}\left[\frac{k_{3}}{k_{1}}-1\right]+S\left\{\left[\frac{k_{3}}{k_{1}}-1\right] a-\frac{\psi}{k_{1}} V_{\max }+1\right\}+a=0 \\
& \Phi_{1}\left(k_{1}, k_{3}, \psi, a\right)=\left\{\left[\frac{k_{3}}{k_{1}}-1\right] a-\frac{\psi^{\prime}}{k_{1}} V_{\max }+1\right\}^{2} \\
& -4\left[\frac{k_{9}}{k_{1}}-1\right] a \\
& S^{(2,3)}=\frac{-\left\{\left[\frac{k_{3}}{k_{1}}-1\right] a-\frac{\psi}{k_{1}} V_{\max }+1\right\}}{ \pm \sqrt{\Phi_{1}\left(k_{1}, k_{3}, \psi, a\right)}} \\
& \psi\left(\begin{array}{c}
-\left\{\left[\frac{k_{3}}{k_{1}}-1\right] a-\frac{\psi}{k_{1}} V_{\max }+1\right\} \\
\psi f\left(\frac{ \pm \sqrt{\Phi_{1}\left(k_{1}, k_{3}, \psi, a\right)}}{2\left[\frac{k_{3}}{k_{1}}-1\right]}\right)
\end{array}\right. \\
& -\left\{\left[\frac{k_{3}}{k_{1}}-1\right] a-\frac{\psi}{k_{1}} V_{\max }+1\right\} \\
& B^{(2,3)}=\frac{-\left(\frac{ \pm \sqrt{\Phi_{1}\left(k_{1}, k_{3}, \psi, a\right)}}{2\left[\frac{k_{3}}{k_{1}}-1\right]}\right) k_{3}}{k_{1}} \\
& \begin{array}{l}
\left.\left\{\left(\left[\frac{k_{3}}{k_{1}}-1\right] a-\frac{\psi}{k_{1}} V_{\max }+1\right)^{2}-4\left[\frac{k_{3}}{k_{1}}-1\right] a\right\}\right|_{k_{1}=k_{3}} \\
=\left(-\frac{\psi}{k_{1}} V_{\max }+1\right)^{2}
\end{array} \\
& \left.\left\{\left[\frac{k_{3}}{k_{1}}-1\right] a-\frac{\psi}{k_{1}} V_{\max }+1\right\}\right|_{k_{1}=k_{3}}=1-\frac{\psi}{k_{1}} V_{\max }
\end{aligned}
$$

The above case can't exist since $\left.\frac{1}{2\left[\frac{k_{3}}{k_{1}}-1\right]}\right|_{k_{1}=k_{s}} \rightarrow \infty$.

The third and fourth fixed points:

$$
\begin{aligned}
& E^{(2,3)}\left(V^{(2,3)}, B^{(2,3)}, S^{(2,3)}\right)
\end{aligned}
$$

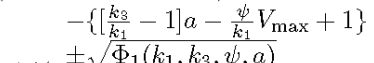

$$
\begin{aligned}
& \psi f\left(\frac{ \pm \sqrt{\Phi_{1}\left(k_{1}, k_{3}, \psi, a\right)}}{2\left[\frac{k_{3}}{k_{1}}-1\right]}\right) \\
& \left.-\left\{\frac{k_{8}}{k_{1}}-1\right] a-\frac{\psi}{k_{1}} V_{\max }+1\right\} \\
& =\left(0, \frac{-\left(\frac{ \pm \sqrt{\Phi_{1}\left(k_{1}, k_{3}, \psi, a\right)}}{2\left[\frac{k_{3}}{k_{1}}-1\right]}\right) k_{3}}{k_{1}}\right. \\
& -\left\{\left[\frac{k_{3}}{k_{1}}-1\right] a-\frac{\psi}{k_{1}} V_{\max }+1\right\} \\
& \left., \frac{ \pm \sqrt{\Phi_{1}\left(k_{1}, k_{3}, \psi, a\right)}}{2\left[\frac{k_{2}}{k_{2}}-1\right]}\right)
\end{aligned}
$$

From the second equation $f_{2}(S, V, B)$ :

$$
V\left\{-\varphi+\gamma B\left(1-\frac{1}{\beta}\right)-\left[B k_{1}+V k_{2}+S k_{3}\right]\right\}=0
$$

Case No.2:

$$
-\varphi+\gamma B\left(1-\frac{1}{\beta}\right)-\left[B k_{1}+V k_{2}+S k_{3}\right]=0
$$

(61)

(69)

$$
\begin{aligned}
& -\varphi+B\left[\gamma\left(1-\frac{1}{\beta}\right)-k_{1}\right]-V k_{2}-S k_{3}=0 \\
& B=\frac{\varphi+V k_{2}+S k_{3}}{\left[\gamma\left(1-\frac{1}{\beta}\right)-k_{1}\right]}
\end{aligned}
$$

Third equation gives:

$$
\begin{aligned}
& \psi f(S) B-\gamma V B-B\left[B k_{1}+V k_{2}+S k_{3}\right]=0 \\
& B\left\{\psi f(S)-\gamma V-\left[B k_{1}+V k_{2}+S k_{3}\right]\right\}=0 \\
& \left.\Rightarrow\right|_{B \neq 0} \psi f(S)-\gamma V-\left[B k_{1}+V k_{2}+S k_{3}\right]=0
\end{aligned}
$$

$$
\psi f(S)-\gamma V-\left[\left(\frac{\varphi+V k_{2}+S k_{3}}{\left[\gamma\left(1-\frac{1}{\beta}\right)-k_{1}\right]}\right) k_{1}+V k_{2}+S k_{3}\right]=0 \quad(75)
$$

$$
\psi f(S)-\gamma V-\left(\frac{\varphi+V k_{2}+S k_{3}}{\left[\gamma\left(1-\frac{1}{\beta}\right)-k_{1}\right]}\right) k_{1}-V k_{2}-S k_{3}=0 \quad(76)
$$

$$
\psi f(S)-\gamma V-\frac{\varphi k_{1}}{\left[\gamma\left(1-\frac{1}{\beta}\right)-k_{1}\right]}-\frac{k_{2} k_{1}}{\left[\gamma\left(1-\frac{1}{\beta}\right)-k_{1}\right]} V
$$$$
-\frac{k_{3} k_{1}}{\left[\gamma\left(1-\frac{1}{\beta}\right)-k_{1}\right]} S-V k_{2}-S k_{3}=0
$$$$
\begin{aligned}
& -\frac{k_{2} k_{1}}{\left[\gamma\left(1-\frac{1}{\beta}\right)-k_{1}\right]} V-\gamma V-V k_{2}+\psi f(S) \\
& -\frac{k_{s} k_{1}}{\left[\gamma\left(1-\frac{1}{\rho}\right)-k_{1}\right]} S-S k_{3}-\frac{\varphi k_{1}}{\left[\gamma\left(1-\frac{1}{b}\right)-k_{1}\right]}=0
\end{aligned}
$$

$$
\begin{aligned}
& -V\left[\frac{k_{2} k_{1}}{\left[\gamma\left(1-\frac{1}{\beta}\right)-k_{1}\right]}+\gamma+k_{2}\right] \\
& +S\left\{\frac{\psi V_{m a x}}{(a+S)}-\frac{k_{3} k_{1}}{\left[\gamma\left(1-\frac{1}{\beta}\right)-k_{1}\right]}-k_{3}\right\}-\frac{\varphi k_{1}}{\left[\gamma\left(1-\frac{1}{\beta}\right)-k_{1}\right]}=0
\end{aligned}
$$

$V\left[\frac{k_{2} k_{1}}{\left[\gamma\left(1-\frac{1}{\beta}\right)-k_{1}\right]}+\gamma+k_{2}\right]$

$=S\left\{\frac{\psi V_{\max }}{(a+S)}-\left(\frac{k_{3} k_{1}}{\left[\gamma\left(1-\frac{1}{\beta}\right)-k_{1}\right]}+k_{3}\right)\right\}-\frac{\varphi k_{1}}{\left[\gamma\left(1-\frac{1}{\beta}\right)-k_{1}\right]}$

We define for simplicity:

$$
\begin{aligned}
& \Gamma_{1}=\frac{k_{2} k_{1}}{\left[\gamma\left(1-\frac{1}{1}\right)-k_{1}\right]}+\gamma+k_{2} \\
& \Gamma_{2}=\frac{k_{3} k_{1}}{\left[\gamma\left(1-\frac{1}{\beta}\right)-k_{1}\right]}+k_{3}
\end{aligned}
$$

We have

$$
\begin{gathered}
B=\frac{\varphi+V k_{2}+S k_{3}}{\left[\gamma\left(1-\frac{1}{\beta}\right)-k_{1}\right]} ; V=S \frac{\left\{\frac{\psi\left(\frac{\psi V_{\max }}{(a+S)}-\Gamma_{2}\right\}}{\Gamma_{1}}-\frac{\Gamma_{3}}{\Gamma_{1}}\right.}{B=\frac{\varphi+V k_{2}+S k_{3}}{\left[\gamma\left(1-\frac{1}{\beta}\right)-k_{1}\right]}} \\
B=\frac{\varphi+\left[S \frac{\left\{\psi\left(\frac{\psi}{(\max )}-\Gamma_{2}\right\}\right.}{\Gamma_{1}}-\frac{\Gamma_{3}}{\Gamma_{1}}\right] k_{2}+S k_{3}}{\left[\gamma\left(1-\frac{1}{\beta}\right)-k_{1}\right]}
\end{gathered}
$$

From first equation:

$$
(1-S)\left(B k_{1}+V k_{2}+S k_{3}\right)-\psi f(S) B=0
$$




$$
\begin{aligned}
& (1-S)\left(\left\{\frac{\varphi+\left[S \frac{\left\{\frac{\left\{\psi_{\max }\right.}{\left(\alpha+S_{1}\right.}-\Gamma_{2}\right\}}{\Gamma_{1}}-\frac{\Gamma_{3}}{\Gamma_{1}}\right] k_{2}+S k_{3}}{\left[\gamma\left(1-\frac{1}{\beta}\right)-k_{1}\right]}\right\} k_{1}\right. \\
& \left.+\left\{S \frac{\left\{\frac{\psi\left(V_{\max }+\alpha_{a}\right)}{\Gamma_{1}}-\Gamma_{2}\right\}}{\Gamma_{1}}-\frac{\Gamma_{3}}{\Gamma_{1}}\right\} k_{2}+S k_{3}\right) \\
& -\psi f(S)\left\{\frac{\varphi+\left[S \frac{\left\{\frac{\left\{V_{\max }\right.}{\left(\alpha+S_{1}\right.}-\Gamma_{2}\right\}}{\Gamma_{1}}-\frac{\Gamma_{3}}{\Gamma_{1}}\right] k_{2}+S k_{8}}{\left[\gamma\left(1-\frac{1}{\beta}\right)-k_{1}\right]}\right\}=0
\end{aligned}
$$

We define for simplicity $\Gamma_{4}=\left[\gamma\left(1-\frac{1}{\beta}\right)-k_{1}\right]$

$$
\begin{aligned}
& (1-S)\left(\left\{\frac{\varphi+\left[S \frac{\left\{\frac{\left\{V_{\max }\right.}{(\alpha+S)}-\Gamma_{2}\right\}}{\Gamma_{1}}-\frac{\Gamma_{8}}{\Gamma_{1}}\right] k_{2}+S k_{3}}{\Gamma_{4}}\right\} k_{1}\right. \\
& \left.+\left\{S \frac{\left\{\frac{\left.4 V_{\text {max }}+T_{0}\right)}{\Gamma_{1}}-\Gamma_{2}\right\}}{\Gamma_{1}}-\frac{\Gamma_{3}}{\Gamma_{1}}\right\} k_{2}+S k_{3}\right) \\
& -\psi f(S)\left\{\frac{\varphi+\left[S \frac{\left\{\frac{\left\{V_{m a x}\right.}{(\alpha+S)}-\Gamma_{2}\right\}}{\Gamma_{1}}-\frac{\Gamma_{3}}{\Gamma_{1}}\right] k_{2}+S k_{3}}{\Gamma_{4}}\right\}=0 \\
& (1-S)\left(\frac{\varphi}{\Gamma_{4}} k_{1}+\left[S \frac{\left\{\frac{\psi V_{\max }}{(\alpha+S)}-\Gamma_{2}\right\}}{\Gamma_{1}}-\frac{\Gamma_{3}}{\Gamma_{1}}\right] \frac{1}{\Gamma_{4}} k_{2} k_{1}\right. \\
& \left.+S \frac{1}{\Gamma_{4}} k_{3} k_{1}+S\left(\frac{\psi V_{\max }}{(a+S)}-\Gamma_{2}\right) \frac{k_{2}}{\Gamma_{1}}-\frac{\Gamma_{3}}{\Gamma_{1}} k_{2}+S k_{3}\right) \\
& -\psi \frac{V_{\max } S}{a+S}\left\{\varphi \frac{1}{\Gamma_{4}}+\left[S \frac{\left\{\frac{\psi\left(V_{\max }\right.}{(a+S)}-\Gamma_{2}\right\}}{\Gamma_{1}}\right.\right. \\
& \left.\left.-\frac{\Gamma_{3}}{\Gamma_{1}}\right] k_{2} \frac{1}{\Gamma_{4}}+S k_{3} \frac{1}{\Gamma_{4}}\right\}=0 ; f(S)=\frac{V_{\max S} S}{a+S}
\end{aligned}
$$

For simplicity we define:

$$
\begin{aligned}
& \iota(S)=\frac{\psi V_{\max } S}{(a+S)} \Rightarrow \iota(S)=\psi f(S) \Rightarrow \psi=\frac{\iota(S)}{f(S)} \\
& \quad(1-S)\left(\frac{\varphi}{\Gamma_{4}} k_{1}+\left[\frac{\left\{\iota(S)-S \Gamma_{2}\right\}}{\left.\Gamma_{1}\right\}}-\frac{\Gamma_{3}}{\Gamma_{1}}\right] \frac{1}{\Gamma_{4}} k_{2} k_{1}\right. \\
& \left.\left.\quad+S \frac{1}{\Gamma_{4}} k_{3} k_{1}+\iota \iota(S)-S \Gamma_{2}\right] \frac{k_{2}}{\Gamma_{1}}-\frac{\Gamma_{3}}{\Gamma_{1}} k_{2}+S k_{3}\right) \\
& \quad-\iota(S)\left\{\varphi \frac{1}{\Gamma_{4}}+\left[\frac{\left\{\iota(S)-S \Gamma_{2}\right)}{\Gamma_{1}}-\frac{\Gamma_{3}}{\Gamma_{1}} k_{2} \frac{1}{\Gamma_{4}}\right.\right. \\
& \left.\quad+S k_{3} \frac{1}{\Gamma_{4}}\right\}=0
\end{aligned}
$$

$-1$

Since the above equation is polynomial shape in $S$ variable, it is easy to solve it numerically than analytic to find fixed point $E^{(i>3)}\left(V^{(i>3)}, B^{(i>3)}, S^{(i>3)}\right) ; V^{(i>3)} \neq 0 ; B^{(i>3)} \neq$ $0 ; S^{(i>3)} \neq 0$. We need to neglect all fixed points numerical solutions which phage, bacteria or nutrient values are negative or complex [4] [5]. We can summery our last results in the next diagram:

$$
\begin{aligned}
& \mathrm{EQ} .1:(1-S)\left(B k_{1}+V k_{\mathscr{Q}}+S k_{8}\right)-\psi f(S) B=0 \\
& \mathrm{EQ.2:} \begin{array}{l}
-\varphi V+\gamma V B\left(1-\frac{1}{\beta}\right)-V\left[B k_{1}+V k_{2}\right. \\
\left.+S k_{3}\right]=0
\end{array}
\end{aligned}
$$

$\mathrm{EQ} .3: \psi f(S) B-\gamma V B-B\left[B k_{1}+V k_{2}+S k_{3}\right]=0$

We already calculate our Chemostat scaled parameters and have the following values: $\gamma=18.74 ; \psi=3.69 ; a=$ $1.81 ; \varphi=\frac{\psi}{2}=1.845 ; \beta=71$. For our Chemostat washout rate balance parameters $k_{i}[\mathrm{ml} / \mathrm{sec}]$.

$$
\begin{aligned}
& V_{M}=1 ; \sum_{i=1}^{3} k_{i}=1 ; \sum_{i=1}^{3} k_{i}=1 \\
& k_{1}=0.2 ; k_{2}=0.3 ; k_{3}=0.5
\end{aligned}
$$

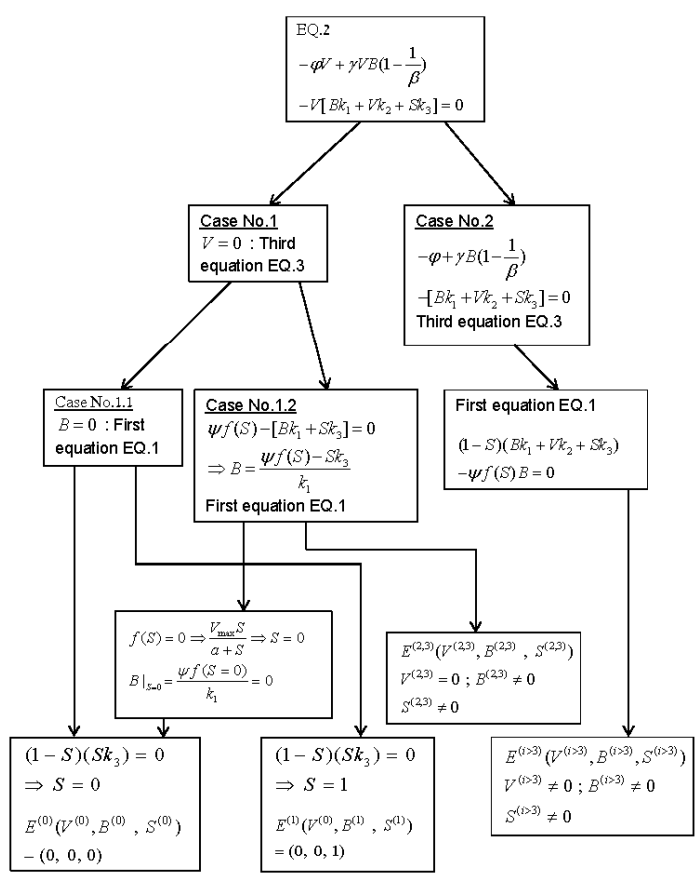

Fig. 2. Chemostat system with dilution control parameter fixed points flow chart.

$$
\begin{aligned}
\Gamma_{1} & =\frac{k_{2} k_{1}}{\left[\gamma\left(1-\frac{1}{6}\right)-k_{1}\right]}+\gamma+k_{2} \\
\Gamma_{1} & =\frac{0.3 \times 0.2}{\left[18.74 \times\left(1-\frac{1}{T_{1}}\right)-0.2\right]}+18.74+0.3=19.04 \\
\Gamma_{2} & =k_{3}\left[\frac{k_{1}}{\left[\gamma\left(1-\frac{1}{\beta}\right)-k_{1}\right]}+1\right] \\
\Gamma_{2} & =0.5 \times\left[\frac{0.2}{\left[18.74 \times\left(1-\frac{1}{\tau 1}\right)-0.2\right]}+1\right]=0.505 \\
\Gamma_{3} & =\frac{\varphi k_{1}}{\left[\gamma\left(1-\frac{1}{\beta}\right)-k_{1}\right]}=\frac{1.845 \times 0.2}{\left[18.74 \times\left(1-\frac{1}{\gamma 1}\right)-0.2\right]}=0.0202 \\
\Gamma_{4} & =\left[\gamma\left(1-\frac{1}{\beta}\right)-k_{1}\right]=18.25
\end{aligned}
$$

The first fixed point: $E^{(0)}\left(V^{(0)}, B^{(0)}, S^{(0)}\right)=(0,0,0)$ The second fixed point: $E^{(1)}\left(V^{(1)}, B^{(1)}, S^{(1)}\right)=(0,0,1)$

The third fixed point: $E^{(2)}\left(V^{(2)}, B^{(2)}, S^{(2)}\right)=(0,-8.63,9.69)$

Only mathematic fixed point, not biologic (bacteria fixed point value is negative

The forth fixed point: $E^{(3)}\left(V^{(3)}, B^{(3)}, S^{(3)}\right)=(0,0.885,0.126)$

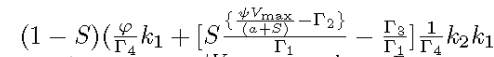

$\left.+S \frac{1}{\Gamma_{4}} k_{3} k_{1}+S\left(\frac{\psi V_{\max }}{(a+S)}-\Gamma_{2}\right) \frac{k_{2}}{\Gamma_{1}}-\frac{\Gamma_{3}}{\Gamma_{1}} k_{2}+S k_{3}\right)$

$-\psi \frac{V_{\max } S}{a+S}\left\{\varphi \frac{1}{\Gamma_{4}}+\left[S \frac{\left\{\frac{\left\langle V_{\max }\right.}{(+9)}-\Gamma_{2}\right\}}{\Gamma_{1}}-\frac{\Gamma_{8}}{\Gamma_{1}}\right] k_{2} \frac{1}{\Gamma_{4}}\right.$ 
We use Mathematica software to solve the above equation (S variable):

$$
\begin{aligned}
& \{\{S=-1.79687+0.13257 i\} \\
& \{\{S=-1.79687-0.13257 i\} \\
& \{S=0.676383\},\{S=-0.0596224\}\}
\end{aligned}
$$

We ignore mathematics results (Nutrient negative and complex values) and take only non-negative nutrient fixed point $(S \rightarrow 0.676383)$.

$$
\begin{gathered}
V=S \frac{\left\{\frac{\psi V_{\max }}{(a+S)}-\Gamma_{2}\right\}}{\Gamma_{1}}-\frac{\Gamma_{3}}{\Gamma_{1}} \\
V=0.676 \times \frac{\left\{\frac{3.69}{(1.81+0.646)}-0.505\right\}}{19.04} \\
-\frac{0.0202}{19.04}=0.0337 \\
B=\frac{\varphi+V k_{2}+S k_{3}}{\left[\gamma\left(1-\frac{1}{8}\right)-k_{1}\right]} \\
B=\frac{1.845+0.0337 \times 0.3+0.676 \times 0.5}{\left[18.74 \times\left(1-\frac{1}{71}\right)-0.2\right]}=0.12
\end{gathered}
$$

The fifth fixed point:

$E^{(4)}\left(V^{(4)}, B^{(4)}, S^{(4)}\right)=(0.0337,0.12,0.676)$. In our final result we count only biological fixed points and neglect all mathematics fixed points. Our biological fixed points are as below.

The first fixed point: $E^{(0)}\left(V^{(0)}, B^{(0)}, S^{(0)}\right)=(0,0,0)$

The second fixed point: $E^{(1)}\left(V^{(1)}, B^{(1)}, S^{(1)}\right)=(0,0,1)$.

The third fixed point: $E^{(2)}\left(V^{(2)}, B^{(2)}, S^{(2)}\right)=(0,0.885,0.126)$.

The forth fixed point:

$E^{(3)}\left(V^{(3)}, B^{(3)}, S^{(3)}\right)=(0.0337,0.12,0.676)$

II. ChEMostat SySTEM With Dilution CONTROL PARAMETER FIXED POINTS CLASSIFICATION AND STABILITY

To classify Chemostat fixed points, we need to compute the Jacobian.

$$
\begin{aligned}
& f_{1}(S, V, B)=(1-S)\left(B k_{1}+V k_{2}+S k_{3}\right)-\psi f(S) B(102) \\
& \quad f_{2}(S, V, B)=-\varphi V+\gamma V B\left(1-\frac{1}{\beta}\right) \\
& \quad-V\left[B k_{1}+V k_{2}+S k_{3}\right] \\
& f_{3}(S, V, B)=\psi f(S) B-\gamma V B-B\left[B k_{1}+V k_{2}+S k_{3}\right]
\end{aligned}
$$

We define $E^{(*)}\left(V^{(*)}, B^{(*)}, S^{(*)}\right)=\left(V^{*}, B^{*}, S^{*}\right)$ as any Chemostat fixed point.

$$
\begin{aligned}
& A=\left(\begin{array}{lll}
\frac{\partial f_{1}}{\partial S} & \frac{\partial f_{1}}{\partial V} & \frac{\partial f_{1}}{\partial P} \\
\frac{\partial f_{2}}{\partial S} & \frac{\partial f_{2}}{\partial V} & \frac{\partial f_{2}}{\partial D} \\
\frac{\partial f_{3}}{\partial S} & \frac{\partial f_{3}}{\partial V} & \frac{\partial f_{3}}{\partial B}
\end{array}\right)_{\left(V^{*}, B^{*}, S^{*}\right)} ; \operatorname{det}|A-\lambda I|=0 \\
& f(S)=\frac{V_{\max } S}{a+S} \Rightarrow \frac{\partial f(S)}{\partial S}=\frac{V_{\max } a}{(a+S)^{2}} \\
& \left.\quad \frac{\partial f_{1}}{\partial S}\right|_{\left(V^{*}, B^{*}, S^{*}\right)}=-\left(B^{*} k_{1}+V^{*} k_{2}+S^{*} k_{3}\right) \\
& \quad+\left(1-S^{*}\right) k_{3}-\psi B^{*} \frac{\partial f\left(S^{*}\right)}{\partial S}
\end{aligned}
$$

$\left.\frac{\partial f_{1}}{\partial S}\right|_{\left(V^{*}, B^{*}, S^{*}\right)}=-\left(B^{*} k_{1}+V^{*} k_{2}+S^{*} k_{3}\right)$

$$
+\left(1-S^{*}\right) k_{3}-\psi B^{*} \frac{V_{\max } a}{\left(a+S^{*}\right)^{2}}
$$

$$
\begin{aligned}
& \left.\frac{\partial f_{1}}{\partial V}\right|_{\left(V^{*}, B^{*}, S^{*}\right)}=\left(1-S^{*}\right) k_{2} \\
& \begin{array}{l}
\left.\frac{\partial Y}{\partial 1}\right|_{\left(V^{*}, B^{*}, S^{*}\right)}=\left(1-S^{*}\right) k_{1}-\psi f\left(S^{*}\right) \\
\left.\frac{\partial f_{2}}{\partial S}\right|_{\left(V^{*}, B^{*}, S^{*}\right)}=-V^{*} k_{3}
\end{array} \\
& \left.\frac{\partial f_{2}}{\partial V}\right|_{\left(V^{*}, B^{*}, S^{*}\right)}=-\varphi+\gamma B^{*}\left(1-\frac{1}{\beta}\right) \\
& -\left(B^{*} k_{1}+V^{*} k_{2}+S^{*} k_{3}\right)-V^{*} k_{2}
\end{aligned}
$$

$$
\begin{aligned}
& \left.\frac{\partial f_{2}}{\partial B}\right|_{\left(V^{*}, B^{*}, S^{*}\right)}=\gamma V^{*}\left(1-\frac{1}{\beta}\right)-V^{*} k_{1} \\
& =V^{*}\left[\gamma\left(1-\frac{1}{\beta}\right)-k_{1}\right] \\
& \left.\frac{\partial f_{3}}{\partial S}\right|_{\left(V^{*}, B^{*}, S^{*}\right)}=\psi B^{*} \frac{\partial f\left(S^{*}\right)}{\partial S}-B^{*} k_{3}
\end{aligned}
$$

$$
\begin{aligned}
& \left.\frac{\partial f_{3}}{\partial S}\right|_{\left(V^{*}, B^{*}, S^{*}\right)}=B^{*}\left[\psi \frac{V_{\max } a}{\left(a+S^{*}\right)^{2}}-k_{3}\right] \\
& \left.\frac{\partial f_{3}}{\partial V}\right|_{\left(V^{*}, B^{*}, S^{*}\right)}=-B^{*}\left(\gamma+k_{2}\right) \\
& \left.\frac{\partial f_{3}}{\partial B}\right|_{\left(V^{+}, B^{+}, S^{*}\right)}=\psi f\left(S^{*}\right)-\gamma V^{*} \\
& -\left(B^{*} k_{1}+V^{*} k_{2}+S^{*} k_{3}\right)-B^{*} k_{1}
\end{aligned}
$$

$\Xi_{11}=\left.\frac{\partial f_{1}}{\partial S}\right|_{\left(V^{*}, B^{*}, S^{*}\right)} ; \Xi_{12}=\left.\frac{\partial f_{1}}{\partial V}\right|_{\left(V^{*}, B^{*}, S^{*}\right)}$ $\Xi_{13}=\left.\frac{\partial f_{1}}{\partial B}\right|_{\left(V^{*}, B^{*}, S^{*}\right)} ; \Xi_{21}=\left.\frac{\partial f_{2}}{\partial S}\right|_{\left(V^{*}, B^{*}, S^{*}\right)}$ $\Xi_{22}=\left.\frac{\partial f_{2}}{\partial V}\right|_{\left(V^{*}, B^{*}, S^{*}\right)}$

$$
\begin{aligned}
& \Xi_{23}=\left.\frac{\partial f_{2}}{\partial P}\right|_{\left(V^{*}, B^{*}, S^{*}\right)} ; \Xi_{31}=\left.\frac{\partial f_{3}}{\partial S}\right|_{\left(V^{*}, B^{*}, S^{*}\right)} \\
& \Xi_{32}=\left.\frac{\partial f_{3}}{\partial V}\right|_{\left(V^{*}, B^{*}, S^{*}\right)} ; \Xi_{33}=\left.\frac{\partial f_{3}}{\partial B}\right|_{\left(V^{*}, B^{*}, S^{*}\right)}
\end{aligned}
$$

The Jacobian is:

$$
\begin{gathered}
A=\left(\begin{array}{ccc}
\Xi_{11} & \Xi_{12} & \Xi_{13} \\
\Xi_{21} & \Xi_{22} & \Xi_{23} \\
\Xi_{31} & \Xi_{32} & \Xi_{33}
\end{array}\right) ; \operatorname{det}|A-\lambda I|=0 \\
A-\lambda I=\left(\begin{array}{ccc}
\Xi_{11}-\lambda & \Xi_{12} & \Xi_{13} \\
\Xi_{21} & \Xi_{22}-\lambda & \Xi_{23} \\
\Xi_{31} & \Xi_{32} & \Xi_{33}-\lambda
\end{array}\right)
\end{gathered}
$$

$\operatorname{det}|A-\lambda I|=0 \Rightarrow\left(\Xi_{11}-\lambda\right)\left[\left(\Xi_{22}-\lambda\right)\left(\Xi_{33}-\lambda\right)\right.$ $\left.-\Xi_{32} \Xi_{23}\right]-\Xi_{12}\left[\Xi_{21}\left(\Xi_{33}-\lambda\right)-\Xi_{31} \Xi_{23}\right]$ $+\Xi_{13}\left[\Xi_{21} \Xi_{32}-\Xi_{31}\left(\Xi_{22}-\lambda\right)\right]$

$\Xi_{11}=\left.\frac{\partial f_{1}}{\partial S}\right|_{\left(V^{*}, B^{*}, S^{*}\right)} ; \Xi_{12}=\left.\frac{\partial f_{1}}{\partial V}\right|_{\left(V^{*}, B^{*}, S^{*}\right)}$ $\Xi_{13}=\left.\frac{\partial f_{1}}{\partial B}\right|_{\left(V^{*}, B^{*}, S^{*}\right)} ; \Xi_{21}=\left.\frac{\partial f_{2}}{\partial S}\right|_{\left(V^{*}, B^{*}, S^{*}\right)}$ $\Xi_{22}=\left.\frac{\partial f_{2}}{\partial V}\right|_{\left(V^{+}, B^{+}, S^{+}\right)}$

$$
\begin{aligned}
& \Xi_{23}=\left.\frac{\partial f_{2}}{\partial P}\right|_{\left(V^{*}, B^{*}, S^{*}\right)} ; \Xi_{31}=\left.\frac{\partial f_{3}}{\partial S}\right|_{\left(V^{*}, B^{*}, S^{*}\right)} \\
& \Xi_{32}=\left.\frac{\partial f_{3}}{\partial V}\right|_{\left(V^{*}, B^{*}, S^{*}\right)} ; \Xi_{33}=\left.\frac{\partial f_{3}}{\partial B}\right|_{\left(V^{*}, B^{*}, S^{*}\right)}
\end{aligned}
$$




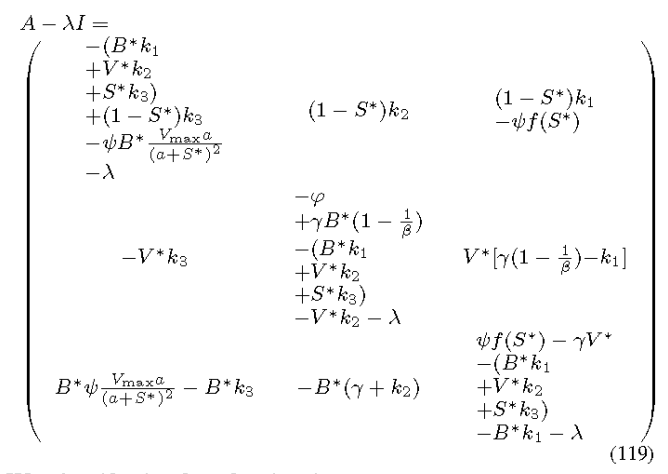

We classify the first fixed point: $E^{(0)}\left(V^{(0)}, B^{(0)}, S^{(0)}\right)=(0,0,0)$

$$
\begin{aligned}
& \left.\frac{\partial f_{1}}{\partial S_{1}}\right|_{\left(V^{*}, B^{*}, S^{*}\right)=(0,0,0)}=k_{3} \\
& \left.\frac{\partial f_{1}}{\partial V}\right|_{\left(V^{*}, B^{*}, S^{*}\right)=(0,0,0)}=k_{2} \\
& \left.\frac{\partial f_{1}}{\partial P}\right|_{\left(V^{*}, B^{*}, S^{*}\right)=(0,0,0)}=k_{1} \\
& \left.\frac{\partial f_{2}}{\partial S}\right|_{\left(V^{*}, B^{*}, S^{*}\right)=0} \\
& \left.\frac{\partial f_{3}}{\partial V}\right|_{\left(V^{*}, B^{*}, S^{*}\right)=(0,0,0)}=-\varphi \\
& \left.\frac{\partial f_{3}}{\partial P}\right|_{\left(V^{*}, B^{*}, S^{+}\right)=(0,0,0)}=0 \\
& \left.\frac{\partial f_{3}}{\partial S}\right|_{\left(V^{*}, B^{*}, S^{*}\right)}=0 \\
& \left.\frac{\partial f_{3}}{\partial S}\right|_{\left(V^{*}, B^{*}, S^{*}\right)=(0,0,0)}=0 \\
& \left.\frac{\partial f_{3}}{\partial V}\right|_{\left(V^{*}, B^{*}, S^{*}\right)=(0,0,0)}=0 \\
& \left.\frac{\partial f_{3}}{\partial B}\right|_{\left(V^{*}, B^{+}, S^{+}\right)=(0,0,0)}=0
\end{aligned}
$$

$\Xi_{11}=\left.\frac{\partial f_{1}}{\partial f_{1}}\right|_{\left(V^{*}, B^{*}, S^{*}\right)} ; \Xi_{12}=\left.\frac{\partial f_{1}}{\partial V_{f}}\right|_{\left(V^{*}, B^{*}, S^{*}\right)}$ $\Xi_{13}=\left.\frac{\partial f_{1}}{\partial B}\right|_{\left(V^{*}, B^{*}, S^{*}\right)} ; \Xi_{21}=\left.\frac{\partial f_{2}}{\partial S}\right|_{\left(V^{*}, B^{*}, S^{*}\right)}$ $\Xi_{22}=\frac{\partial f_{2}}{\partial V} \mid\left(V^{*}, B^{*}, S^{*}\right)$

$\Xi_{23}=\left.\frac{\partial f_{2}}{\partial P}\right|_{\left(V^{*}, B^{*}, S^{*}\right)} ; \Xi_{31}=\left.\frac{\partial f_{3}}{\partial S}\right|_{\left(V^{*}, B^{*}, S^{*}\right)}$ $\Xi_{32}=\left.\frac{\partial f_{3}}{\partial V}\right|_{\left(V^{+}, B^{+}, S^{+}\right)} ; \Xi_{33}=\left.\frac{\partial f_{3}}{\partial B}\right|_{\left(V^{+}, B^{+}, S^{+}\right)}$

$A-\left.\lambda I\right|_{\left(V^{*}, B^{*}, S^{*}\right)=(0,0,0)}=\left(\begin{array}{ccc}k_{3}-\lambda & k_{2} & k_{1} \\ 0 & -\varphi-\lambda & 0 \\ 0 & 0 & -\lambda\end{array}\right)$ $\operatorname{det}|A-\lambda I|_{\left(V^{+}, B^{+}, S^{+}\right)=(0,0,0)}=0$

$$
\begin{gathered}
\operatorname{det}|A-\lambda I|_{\left(V^{*}, B^{*}, S^{*}\right)=(0,0,0)}=0 \\
\left(k_{3}-\lambda\right)(\varphi+\lambda) \lambda-k_{2} \operatorname{det}\left(\begin{array}{cc}
0 & 0 \\
0 & -\lambda
\end{array}\right) \\
+k_{1} \operatorname{det}\left(\begin{array}{cc}
0 & -\varphi-\lambda \\
0 & 0
\end{array}\right)=0 \\
\left(k_{3}-\lambda\right)(\varphi+\lambda) \lambda=0 \Rightarrow \lambda_{1}=k_{3} \\
\lambda_{2}=-\varphi ; \lambda_{3}=0 ; k_{3}>0 ; \varphi>0
\end{gathered}
$$

Then the first fixed point is a saddle point We classify the second fixed point: $E^{(1)}\left(V^{(1)}, B^{(1)}, S^{(1)}\right)=(0,0,1)$ $\left.\frac{\partial f_{1}}{\partial S}\right|_{\left(V^{*}, B^{*}, S^{*}\right)=(0,0,1)}=-k_{3} ; f(S=1)=\frac{V_{\max }}{a+1}$

$\left.\frac{\partial f_{1}}{\partial V}\right|_{\left(V^{+}, B^{+}, S^{+}\right)=(0,0,1)}=0$

$\left.\frac{\partial f_{1}}{\partial B}\right|_{\left(V^{*}, B^{*}, S^{*}\right)=(0,0,1)}=-\psi \frac{V_{M A}}{a+1}$

$\left.\frac{\partial f_{1}}{\partial Y_{2}}\right|_{\left(V^{*}, B^{*}, S^{+}\right)}=(0,0,1)=0$
$\frac{\partial f_{2}}{\partial f_{1}}\left(V^{*}, B^{*}, S^{*}\right)=(0,0,1)=0$

$\left.\frac{\partial f_{1}}{\partial B}\right|_{\left(V^{*}, B^{*}, S^{*}\right)=(0,0,1)}=-\psi f\left(S^{*}=1\right)=-\psi \frac{V_{M}}{a+1}$

$\left.\frac{\partial f_{2}}{\partial V}\right|_{\left(V^{*}, B^{*}, S^{*}\right)=(0,0,1)}=-\varphi-k_{3}$

$\left.\frac{\partial f_{2}}{\partial B}\right|_{\left(V^{*}, B^{*}, S^{*}\right)-(0,0,1)}=0$

$\left.\frac{\partial f_{3}}{\partial S}\right|_{\left(V^{*}, B^{*}, S^{*}\right)=(0,0,1)}=0$

$\left.\frac{\partial f_{3}}{\partial S}\right|_{\left(V^{*}, B^{*}, S^{*}\right)=(0,0,1)}=0$

$\left.\frac{\partial f_{3}}{\partial V}\right|_{\left(V^{+}, B^{+}, S^{+}\right)=(0,0,1)}=0$

$\left.\frac{\partial f_{3}}{\partial B}\right|_{\left(V^{*}, B^{*}, S^{*}\right)=(0,0,1)}=\psi \frac{V_{\max }}{a+1}-k_{3}$

\begin{tabular}{|c|c|c|}
\hline $\begin{array}{l}\text { Fixed } \\
\text { point }\end{array}$ & $\begin{array}{l}\text { Eigenvalues } \\
\text { conditions }\end{array}$ & $\begin{array}{l}\text { Parameters } \\
\text { conditions }\end{array}$ \\
\hline $\begin{array}{l}\text { Stable } \\
\text { node }\end{array}$ & $\begin{array}{l}\lambda_{1}<0 \\
\& \lambda_{2}<0 \\
\& \lambda_{3}<0\end{array}$ & $\begin{array}{l}k_{3}>0 ; \varphi+k_{3}>0 \\
\psi \frac{V_{\max }}{a+1}<k_{3} \\
\text { Can't exist for our } \\
\text { parameters values. }\end{array}$ \\
\hline $\begin{array}{l}\text { Unstable } \\
\text { node }\end{array}$ & $\begin{array}{l}\lambda_{1}>0 \\
\& \lambda_{2}>0 \\
\& \lambda_{3}>0\end{array}$ & $\begin{array}{l}k_{3}<0 ; \varphi+k_{3}<0 \\
\psi \frac{V_{\max }}{a+1}>k_{3} \\
\text { Can't exist for our } \\
\text { parameters values. }\end{array}$ \\
\hline
\end{tabular}

$\Xi_{11}=\left.\frac{\partial f_{1}}{\partial S}\right|_{\left(V^{+}, B^{+}, S^{+}\right)} ; \Xi_{12}=\left.\frac{\partial f_{1}}{\partial V}\right|_{\left(V^{+}, B^{+}, S^{+}\right)}$

$\Xi_{13}=\left.\frac{\partial f_{1}}{\partial B}\right|_{\left(V^{*}, B^{*}, S^{*}\right)} ; \Xi_{21}=\left.\frac{\partial f_{2}}{\partial S}\right|_{\left(V^{*}, B^{*}, S^{*}\right)}$

$\Xi_{22}=\left.\frac{\partial f_{2}}{\partial V}\right|_{\left(V^{+}, B^{+}, S^{+}\right)}$

$\Xi_{23}=\left.\frac{\partial f_{2}}{\partial D}\right|_{\left(V^{*}, B^{*}, S^{*}\right)} ; \Xi_{31}=\left.\frac{\partial f_{3}}{\partial S}\right|_{\left(V^{*}, B^{*}, S^{*}\right)}$

$\Xi_{32}=\left.\frac{\partial f_{3}}{\partial V}\right|_{\left(V^{*}, B^{*}, S^{*}\right)} ; \Xi_{33}=\left.\frac{\partial f_{3}}{\partial B}\right|_{\left(V^{*}, B^{*}, S^{*}\right)}$

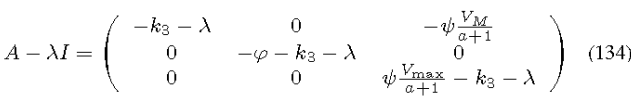

$\operatorname{det}|A-\lambda I|=\left(k_{3}+\lambda\right)\left(\varphi+k_{3}+\lambda\right)\left(\psi \frac{V_{\max }}{a+1}-k_{3}-\lambda\right)$

We get three eigenvalues:

$$
\lambda_{1}=-k_{3} ; \lambda_{2}=-\left(\varphi+k_{3}\right) ; \lambda_{3}=\psi \frac{V_{\max }}{a+1}-k_{3}
$$

Table 1a. Chemostat system with dilution control parameter fixed points and eigenvalues conditions.

(127) We already calculate our chemostat scaled parameters in and have the following values:

$$
\begin{aligned}
& \gamma=18.74 ; \psi=3.69 ; a=1.81 \\
& \varphi=\frac{\psi}{2}=1.845 ; \beta=71
\end{aligned}
$$


Ofer Aluf; Dynamic of Bacterial and Phage Populations Maintained in Chemostat System with Dilution Control Parameter, Transactions on Networks and Communications, Volume 4 No. 3, June (2016); pp: 1-21

For our Chemostat washout rate balance parameters $k_{i}[\mathrm{ml} / \mathrm{sec}]$ we choose $V_{M}=1 ; \sum_{i=1}^{3} k_{i}=1$

$$
\begin{aligned}
& \sum_{i=1}^{3} k_{i}=1 \Rightarrow k_{1}=0.2 ; k_{2}=0.3 ; k_{3}=0.5 \\
& \left(\psi \frac{V_{\max }}{a+1}=1.31\right)>\left(k_{3}=0.5\right) .
\end{aligned}
$$

\begin{tabular}{|c|c|c|}
\hline $\begin{array}{l}\text { Fixed } \\
\text { point } \\
\text { status }\end{array}$ & $\begin{array}{l}\text { Eigenvalues } \\
\text { conditions }\end{array}$ & $\begin{array}{l}\text { Parameters } \\
\text { conditions }\end{array}$ \\
\hline $\begin{array}{l}\text { Saddle } \\
\text { point }\end{array}$ & $\begin{array}{l}\lambda_{1}<0 \\
\& \lambda_{2}<0 \\
\& \lambda_{3}>0\end{array}$ & $\begin{array}{l}\quad k_{3}>0 ; \varphi+k_{3}>0 \\
\psi \frac{V_{\max }}{a+1}>k_{\mathrm{S}} \\
\text { Can exist. }\end{array}$ \\
\hline $\begin{array}{l}\text { Saddle } \\
\text { point }\end{array}$ & $\begin{array}{l}\lambda_{1}<0 \\
\& \lambda_{2}>0 \\
\& \lambda_{3}<0\end{array}$ & $\begin{array}{l}\quad k_{3}>0 ; \varphi+k_{3}<0 \\
\psi \frac{V_{\max }}{a+1}<k_{3} \\
\text { Can't exist for our } \\
\text { parameters values. }\end{array}$ \\
\hline $\begin{array}{l}\text { Saddle } \\
\text { point }\end{array}$ & $\begin{array}{l}\lambda_{1}>0 \\
\& \lambda_{2}<0 \\
\& \lambda_{3}<0\end{array}$ & $\begin{array}{l}\quad k_{3}<0 ; \varphi+k_{3}>0 \\
\psi \frac{V_{\max }}{a+1}<k_{3} \\
\text { Can't exist for our } \\
\text { parameters values. }\end{array}$ \\
\hline
\end{tabular}

Table 1b. Chemostat system with dilution control parameter fixed points

\begin{tabular}{|c|c|c|}
\hline $\begin{array}{l}\text { Fixed } \\
\text { point } \\
\text { status }\end{array}$ & $\begin{array}{l}\text { Eigenvalues } \\
\text { conditions }\end{array}$ & $\begin{array}{l}\text { Parameters } \\
\text { conditions }\end{array}$ \\
\hline $\begin{array}{l}\text { Saddle } \\
\text { point }\end{array}$ & $\begin{array}{l}\lambda_{1}>0 \\
\& \lambda_{2}>0 \\
\& \lambda_{3}<0\end{array}$ & $\begin{array}{l}k_{3}<0 ; \varphi+k_{3}<0 \\
\psi \frac{V_{\max }}{a+1}<k_{3} \\
\text { Can't exist for our } \\
\text { parameters values. }\end{array}$ \\
\hline $\begin{array}{l}\text { Saddle } \\
\text { point }\end{array}$ & $\begin{array}{l}\lambda_{1}>0 \\
\& \lambda_{2}<0 \\
\& \lambda_{3}>0\end{array}$ & $\begin{array}{l}k_{3}<0 ; \varphi+k_{3}>0 \\
\psi \frac{V_{\max }}{a+1}>k_{3} \\
\text { Can’t exist for our } \\
\text { parameters values. }\end{array}$ \\
\hline $\begin{array}{l}\text { Saddle } \\
\text { point }\end{array}$ & $\begin{array}{l}\lambda_{1}<0 \\
\& \lambda_{2}>0 \\
\& \lambda_{3}>0\end{array}$ & $\begin{array}{l}k_{3}>0 ; \varphi+k_{3}<0 \\
\psi \frac{V_{\max }}{a+1}>k_{3} \\
\text { Can't exist for our } \\
\text { parameters values. }\end{array}$ \\
\hline
\end{tabular}
and eigenvalues conditions.

Table 1c. Chemostat system with dilution control parameter fixed points and eigenvalues conditions.

Second fixed point is a saddle point. We classify the third fixed point: $E^{(2)}\left(V^{(2)}, B^{(2)}, S^{(2)}\right)=(0,0.885,0.126)$.

$$
\begin{aligned}
& \left.\frac{\partial f_{1}}{\partial S}\right|_{\left(V^{+}, B^{+}, S^{+}\right)=(0,0.885,0.126)} \\
& =-\left(0.885 \times k_{1}+0.126 \times k_{3}\right)+(1-0.126) \times k_{3} \\
& -3.69 \times 0.885 \times \frac{1.81}{(1.81+0.126)^{2}} \\
& \left.\quad \frac{\partial f_{1}}{\partial S}\right|_{\left(V^{*}, B^{*}, S^{*}\right)=(0,0.885,0.126)} \\
& \quad=0.748 \times k_{3}-0.885 \times k_{1}-1.58 \\
& \left.\quad \frac{\partial f_{1}}{\partial V}\right|_{\left(V^{*}, B^{*}, S^{*}\right)=(0,0.885,0.126)}=0.874 \times k_{2}
\end{aligned}
$$

$\left.\frac{\partial f_{3}}{\partial S}\right|_{\left(V^{*}, B^{*}, S^{*}\right)=(0,0.885,0.126)}$

\begin{tabular}{|c|c|c|}
\hline $\begin{array}{l}\text { A matrix } \\
\text { element }\end{array}$ & $\begin{array}{l}\text { A matrix } \\
\text { element } \\
\text { Function of } k_{i}\end{array}$ & $\begin{array}{l}\text { A-Matrix } \\
\text { element } \\
\text { values for } \\
\sum_{i=1}^{3} k_{i}=1 \\
k_{1}=0.2 \\
k_{2}=0.3 \\
k_{3}=0.5\end{array}$ \\
\hline $\begin{aligned}\left.\frac{\partial f_{1}}{\partial S}\right|_{\left(V^{*}, B^{*}, S^{*}\right)} & =(0,0.885,0.126)\end{aligned}$ & $\begin{array}{l}0.748 \times k_{3} \\
-0.885 \times k_{1}-1.58\end{array}$ & -1.383 \\
\hline $\begin{aligned}\left.\frac{\partial f_{1}}{\partial V}\right|_{\left(V^{+}, B^{+}, S^{+}\right)} & =(0,0.885,0.126)\end{aligned}$ & $0.874 \times k_{2}$ & 0.262 \\
\hline $\begin{array}{l}\frac{\partial f_{1}^{\prime}}{\partial B} \mid\left(V^{+}, B^{+}, S^{+}\right) \\
=(0,0.885,0.126)\end{array}$ & $0.874 \times k_{1}-0.24$ & -0.0652 \\
\hline $\begin{array}{l}\frac{\partial f_{2}}{\partial S} \mid \\
\left(V^{*}, B^{*}, S^{*}\right) \\
=(0,0.885,0.126)\end{array}$ & 0 & 0 \\
\hline $\begin{array}{c}\frac{\partial f_{2}}{\partial V} \mid \\
\quad\left(V^{*}, B^{*}, S^{*}\right) \\
=(0,0.885,0.126)\end{array}$ & $\begin{array}{l}14.49-0.885 \times k_{1} \\
-0.126 \times k_{3}\end{array}$ & 14.25 \\
\hline $\begin{aligned}\left.\frac{\partial f_{2}}{\partial B}\right|_{(} & \left(V^{+}, B^{+}, S^{+}\right) \\
& =(0,0.885,0.126)\end{aligned}$ & 0 & 0 \\
\hline $\begin{aligned} \frac{\partial f_{3}}{\partial S} \mid & \left(V^{*}, B^{*}, S^{*}\right) \\
& =(0,0.885,0.126)\end{aligned}$ & $\begin{array}{l}0.885 \times \\
{\left[1.77-k_{3}\right]}\end{array}$ & 1.12 \\
\hline $\begin{array}{l}\frac{\partial f_{3}}{\partial V} \mid \\
\left(V^{*}, B^{*}, S^{*}\right) \\
\quad=(0,0.885,0.126)\end{array}$ & $\begin{array}{l}-0.885 \times \\
\left(18.74+k_{2}\right)\end{array}$ & -16.85 \\
\hline $\begin{aligned} \frac{\partial f_{3}}{\partial B} \mid & \left(V^{*}, B^{*}, S^{*}\right) \\
& =(0,0.885,0.126)\end{aligned}$ & $\begin{array}{l}0.24-1.77 \times k_{1} \\
-0.126 \times k_{3}\end{array}$ & -0.177 \\
\hline
\end{tabular}

$=B^{*}\left[\psi \frac{V_{\max } a}{\left(a+S^{*}\right)^{2}}-k_{3}\right]=0.885 \times\left[1.77-k_{3}\right]$

$\left.\frac{\partial f_{3}}{\partial V}\right|_{\left(V^{*}, B^{*}, S^{*}\right)=(0,0.885,0.126)}=-0.885 \times\left(18.74+k_{2}\right)$

$\left.\frac{\partial f_{3}}{\partial B}\right|_{\left(V^{*}, B^{*}, S^{*}\right)=(0,0.885,0.126)}$

$=\psi f\left(S^{*}\right)-2 B^{*} k_{1}-S^{*} k_{3}$

$=0.24-1.77 \times k_{1}-0.126 \times k_{3}$

Table 2. Chemostat system with dilution control parameter A matrix (140) elements as a function of $k_{i}$. 


$$
\begin{gathered}
A-\lambda I=\left(\begin{array}{ccc}
\Xi_{11}-\lambda & \Xi_{12} & \Xi_{13} \\
\Xi_{21} & \Xi_{22}-\lambda & \Xi_{23} \\
\Xi_{31} & \Xi_{32} & \Xi_{33}-\lambda
\end{array}\right) \\
=\left(\begin{array}{ccc}
-1.383-\lambda & 0.262 & -0.0652 \\
0 & 14.25-\lambda & 0 \\
1.12 & -16.85 & -0.177-\lambda
\end{array}\right) \\
\quad \operatorname{det}|A-\lambda I|=-(1.383+\lambda) \\
\quad \operatorname{det}\left(\begin{array}{cc}
14.25-\lambda & 0 \\
-16.85 & -(0.177+\lambda) \\
0 & 14.25-\lambda \\
1.12 & -16.85
\end{array}\right)
\end{gathered}
$$

det $|A-\lambda I|=(1.383+\lambda)(14.25-\lambda)(0.177+\lambda)$ $+0.0652 \times 1.12 \times(14.25-\lambda)$

$\operatorname{det}|A-\lambda I|=(1.383+\lambda)(14.25-\lambda)(0.177+\lambda)$ $+0.073(14.25-\lambda) \Rightarrow \operatorname{det}|A-\lambda I|=0$

$$
\lambda_{1}=-1.31802 ; \lambda_{2}=-0.240978 ; \lambda_{3}=14.25
$$

Third fixed point is a saddle point

$$
\begin{aligned}
& \Xi_{11}=\left.\frac{\partial f_{1}}{\partial S}\right|_{\left(V^{*}, B^{*}, S^{*}\right)} ; \Xi_{12}=\left.\frac{\partial f_{1}}{\partial V}\right|_{\left(V^{*}, B^{*}, S^{*}\right)} \\
& \Xi_{13}=\left.\frac{\partial f_{1}}{\partial P}\right|_{\left(V^{*}, B^{+}, S^{+}\right)} ; \Xi_{21}=\left.\frac{\partial f_{2}}{\partial S}\right|_{\left(V^{*}, B^{+}, S^{+}\right)} \\
& \Xi_{22}=\left.\frac{\partial f_{2}}{\partial V}\right|_{\left(V^{*}, B^{*}, S^{*}\right)} \\
& \Xi_{23}=\left.\frac{\partial f_{2}}{\partial D}\right|_{\left(V^{*}, B^{*}, S^{*}\right)} ; \Xi_{31}=\left.\frac{\partial f_{3}}{\partial S}\right|_{\left(V^{*}, B^{*}, S^{*}\right)} \\
& \Xi_{32}=\left.\frac{\partial f_{3}}{\partial V}\right|_{\left(V^{+}, B^{+}, S^{+}\right)} ; \Xi_{33}=\left.\frac{\partial f_{3}}{\partial B}\right|_{\left(V^{+}, B^{+}, S^{+}\right)}
\end{aligned}
$$

We classify the forth fixed point:

$E^{(3)}\left(V^{(3)}, B^{(3)}, S^{(3)}\right)=(0.0337,0.12,0.676)$.

$$
\begin{aligned}
& \left.\frac{\partial f_{1}}{\partial S}\right|_{\left(V^{*}, B^{*}, S^{*}\right)}=-\left(B^{*} k_{1}+V^{*} k_{2}+S^{*} k_{3}\right) \\
& \quad=(0.0337,0.12,0.676) \\
& +\left(1-S^{*}\right) k_{3}-\psi B^{*} \frac{V_{\max } a}{\left(a+S^{*}\right)^{2}}
\end{aligned}
$$

$\underset{\substack{\partial f_{1} \\ \partial S}}{\underset{\left(V^{*}, B^{*}, S^{*}\right)}{(0.0337,0.12,0.676)}}=-(0.12 \times 0.2+0.0337 \times 0.3$ $+0.676 \times 0.5)+(1-0.676) \times 0.5$

$-3.69 \times 0.12 \times \frac{1.81}{(1.81+0.676)^{2}}=-0.339$

$\left.\frac{\partial f_{1}}{\partial V}\right|_{\left(V^{*}, B^{*}, S^{*}\right)=(0.0337,0.12,0.676)}=\left(1-S^{*}\right) k_{2}=0.0972$ $\left.\frac{\partial f_{1}}{\partial B}\right|_{\left(V^{*}, B^{*}, S^{*}\right)=(0.0337,0.12,0.676)}=\left(1-S^{*}\right) k_{1}-\psi f\left(S^{*}\right)$ $=(1-0.676) \times 0.2-3.69 \times 0.271=-0.925$ $f\left(S^{*}=0.676\right)=\frac{0.676}{1.81+0.676}=0.271$

$\frac{\partial f_{2}}{\partial S} \mid\left(V^{*}, B^{*}, S^{*}\right)$

$\begin{aligned} \partial S & \left(V^{*}, B^{*}, S^{*}\right) \\ = & (0.0337,0.12,0.676)\end{aligned}$

$\begin{aligned} & =(0.0337,0.12,0.676) \\ = & -V^{*} k_{3}=-0.0337\end{aligned} \times 0.5=-0.0168$ $\left.\frac{\partial f_{2}}{\partial V}\right|_{\left(V^{*}, B^{*}, S^{*}\right)=(0.0337,0.12,0.676)}=-\varphi+\gamma B^{*}\left(1-\frac{1}{\beta}\right)$

$-\left(B^{*} k_{1}+V^{*} k_{2}+S^{*} k_{3}\right)-V^{*} k_{2}$

$\left.\frac{\partial f_{2}}{\partial V}\right|_{\left(V^{+}, B^{+}, S^{+}\right)}=-1.845+18.74 \times 0.12 \times\left(1-\frac{1}{71}\right)$

$-(0.12 \times 0.2+0.0337 \times 0.3+0.676 \times 0.5)$

$-0.0337 \times 0.3=-0.017$

$\left.\frac{\partial f_{2}}{\partial B}\right|_{\left(V^{*}, B^{*}, S^{*}\right)=(0.0337,0.12,0.676)}=V^{*}\left[\gamma\left(1-\frac{1}{\beta}\right)-k_{1}\right]$

$=0.0337 \times\left[18.74 \times\left(1-\frac{1}{71}\right)-0.2\right]=0.615$

$\left.\frac{\partial f(S)}{\partial S}\right|_{S^{*}=0.676}=\frac{1.81}{(1.81+0.676)^{2}}=0.292$

$\left.\frac{\partial f_{3}}{\partial S}\right|_{\left(V^{+}, B^{+}, S^{+}\right)=(0.0337,0.12,0.676)}=B^{*}\left[\psi \frac{\partial f\left(S^{*}\right)}{\partial S}-k_{3}\right]$

$=0.12 \times[3.69 \times 0.292-0.5]=0.0692$

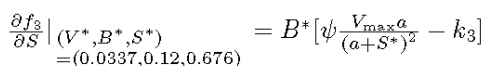

$=0.12 \times\left[3.69 \times \frac{1.81}{(1.81+0.676)^{2}}-0.5\right]=0.069$

$\left.\frac{\partial f_{3}}{\partial V}\right|_{\left(V^{*}, B^{*}, S^{*}\right)}=-B^{*}\left(\gamma+k_{2}\right)$

$=-0.12 \times(18.74+0.3)=-2.248$

$$
\begin{aligned}
& \left.\frac{\partial f_{3}}{\partial B}\right|_{\left(V^{*}, B^{*}, S^{*}\right)}=\psi f\left(S^{*}\right)-\gamma V^{*} \\
& =(0.0337,0.12,0.676) \\
& -\left(B^{*} k_{1}+V^{*} k_{2}+S^{*} k_{3}\right)-B^{*} k_{1}
\end{aligned}
$$

$\left.\frac{\partial f_{3}}{\partial B}\right|_{\left(V^{*}, B^{*}, S^{*}\right)}=3.69 \times 0.271-18.74 \times 0.0337$

$-(0.12 \times 0.2+0.0337 \times 0.3+0.676 \times 0.5)$

$-0.12 \times 0.2=-0.037$

$f\left(S^{*}=0.676\right)=\frac{0.676}{1.81+0.676}=0.271$

$\Xi_{11}=\left.\frac{\partial f_{1}}{\partial S}\right|_{\left(V^{*}, B^{*}, S^{*}\right)}=-0.339$
$\Xi_{12}=\left.\frac{\partial f_{1}}{\partial V}\right|_{\left(V^{*}, B^{*}, S^{*}\right)}=0.0972$
$\Xi_{13}=\left.\frac{\partial f_{1}}{\partial B}\right|_{\left(V^{*}, B^{*}, S^{*}\right)}=-0.925$
$\Xi_{21}=\left.\frac{\partial f_{2}}{\partial S}\right|_{\left(V^{*}, B^{*}, S^{*}\right)}=-0.0168$
$\Xi_{22}=\left.\frac{\partial f_{2}}{\partial V_{2}}\right|_{\left(V^{*}, B^{*}, S^{*}\right)}=-0.017$
$\Xi_{23}=\left.\frac{\partial f_{2}}{\partial B}\right|_{\left(V^{*}, B^{*}, S^{*}\right)}=0.615$

$$
\begin{aligned}
& \Xi_{31}=\left.\frac{\partial f_{3}}{\partial S}\right|_{\left(V^{*}, B^{*}, S^{*}\right)}=0.069 \\
& \Xi_{32}=\left.\frac{\partial f_{3}}{\partial V}\right|_{\left(V^{*}, B^{*}, S^{*}\right)}=-2.248 \\
& \Xi_{33}=\left.\frac{\partial f_{3}}{\partial B}\right|_{\left(V^{*}, B^{*}, S^{*}\right)}=-0.037
\end{aligned}
$$

$A-\lambda I=\left(\begin{array}{ccc}-0.339-\lambda & 0.0972 & -0.925 \\ -0.0168 & -0.017-\lambda & 0.615 \\ 0.069 & -2.248 & -0.037-\lambda\end{array}\right)$
$\operatorname{det}|A-\lambda I|=0$ 


$$
\begin{aligned}
& \operatorname{det}|A-\lambda I|=-(0.339+\lambda) \\
& \operatorname{det}\left(\begin{array}{cc}
-(0.017+\lambda) & 0.615 \\
-2.248 & -(0.037+\lambda)
\end{array}\right) \\
& -0.0972 \times \operatorname{det}\left(\begin{array}{cc}
-0.0168 & 0.615 \\
0.069 & -(0.037+\lambda)
\end{array}\right) \\
& -0.925 \times \operatorname{det}\left(\begin{array}{cc}
-0.0168 & -(0.017+\lambda) \\
0.069 & -2.248
\end{array}\right) \\
& \lambda \rightarrow l
\end{aligned}
$$$$
\operatorname{det}|A-\lambda I|=-(0.339+\lambda)[(0.017+\lambda)(0.037+\lambda)
$$$$
+2.248 \times 0.615]-0.0972 \times[0.0168 \times(0.037+\lambda)
$$$$
-0.069 \times 0.615]-0.925 \times[0.0168 \times 2.248
$$$$
+0.069 \times(0.017+\lambda)]=0 ; \lambda \rightarrow l
$$

$$
\operatorname{det}|A-\lambda I|
$$$$
=-(0.339+\lambda)[(0.017+\lambda)(0.037+\lambda)+1.382]
$$

$-0.0972 \times[0.0168 \times(0.037+\lambda)-0.0424]$

$-0.925 \times[0.0377+0.069 \times(0.017+\lambda)]=0$

$$
\lambda \rightarrow l
$$

Out: $\{\{1=-0.345268\},\{1=-0.0238662-1.20389 i\}$, $\{1=-0.0238662+1.20389 i\}\}$.

Forth fixed point is a stable spiral. Remark: washout rate balance parameters $\left(k_{i}\right)$ establish the classification of each Chemostat's fixed point. We need to plot our Chemosta system 3D diagram for different values of washout rate balance parameters $\left(k_{i}\right)[6][7]$.

MATLAB script:

$$
\begin{aligned}
& V \rightarrow x(1) ; B \rightarrow x(2) ; S \rightarrow x(3) \\
& X \rightarrow x(1) ; Y \rightarrow x(2) ; Z \rightarrow x(3)
\end{aligned}
$$

Remark: There is a Basin Of Attraction (BOA) area surrounding forth fixed point (stable spiral). In the last two figures we choose initial values for bacteria, phage and nutrient within $\mathrm{BOA}$ area, all attracting values are positive and fit our forth fixed point coordinate values.

$$
\begin{aligned}
& E^{(3)}\left(V^{(3)}, B^{(3)}, S^{(3)}\right)=(0.0337,0.12,0.676) \\
& V \rightarrow X ; B \rightarrow Y ; S \rightarrow Z
\end{aligned}
$$

III. Chemostat SYSTEM With CONTROL PARAMETER AND DELAYED VARIABLES ANALYSIS

Our system chemostat (1compartment gradostat) with control variables un-scaled equations: $\tau \neq 0 ; \tau>0$.

$$
\begin{aligned}
& \frac{d V(t)}{d t}=-\varphi V(t)-\gamma V(t) B(t) \\
& +\beta \gamma V(t-\tau) B(t-\tau)-V(t) D \\
& \frac{d B(t)}{d t}=\psi f(S) B(t)-\gamma V(t) B(t)-B(t) D \\
& \frac{d S(t)}{d t}=[S(0)-S(t)] D-\psi f(S) B(t) / \delta \\
& D=\xi(B, V, S)=B k_{1}+V k_{2}+S k_{3} \\
& \quad V=V(t) ; B=B(t) ; S=S(t)
\end{aligned}
$$

The scaled differential equations:

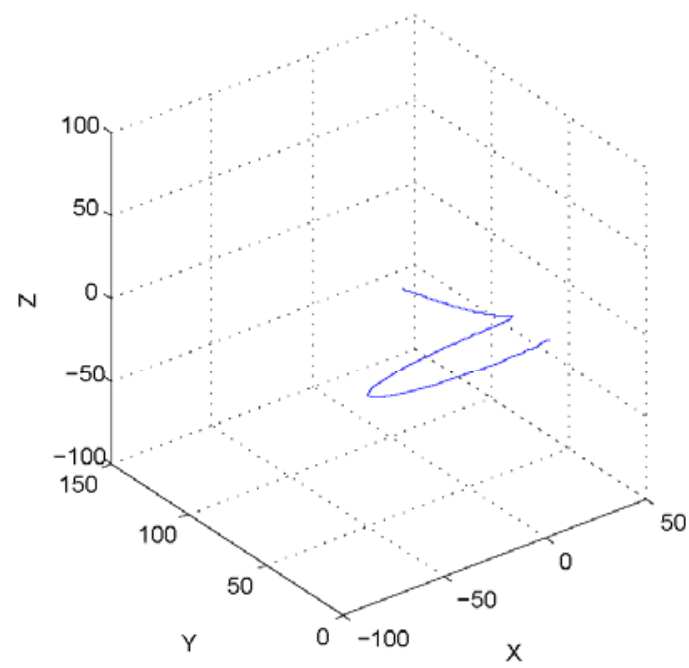

Fig. 3. Chemostat system with dilution control parameter $Z$ v $Y$ vs $X$ for $k_{1}=0.2 ; k_{2}=0.3 ; k_{3}=0.5$.
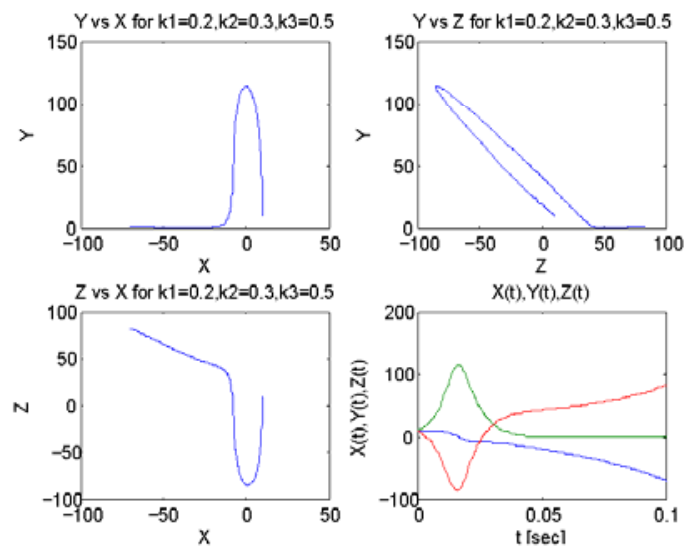

Fig. 4. Chemostat system with dilution control parameter variables phase spaces and time behavior for $k_{1}=0.2 ; k_{2}=0.3 ; k_{3}=0.5$.

$$
\begin{aligned}
& \frac{d V}{d t}=-\varphi V-\gamma V B \\
& +\beta \gamma V(t-\tau) B(t-\tau)-V(t) D \\
& \frac{d B}{d t}=\psi f(S) B-\gamma V B-B D \\
& \frac{d S}{d t}=[S(0)-S] D-\psi f(S) B / \delta \\
& D=\xi(B, V, S)=B k_{1}+V k_{2}+S k_{3}
\end{aligned}
$$

Scaling of the above equation: All resources densities in term of $S^{(0)}$

$$
\begin{aligned}
& \frac{S}{S(0)} \rightarrow S ; \frac{V}{S(0) \beta \delta} \rightarrow V ; \frac{B}{S^{(0)} \delta} \rightarrow B \\
& a \rightarrow \frac{a}{S^{(0)}} ; t \rightarrow t D
\end{aligned}
$$




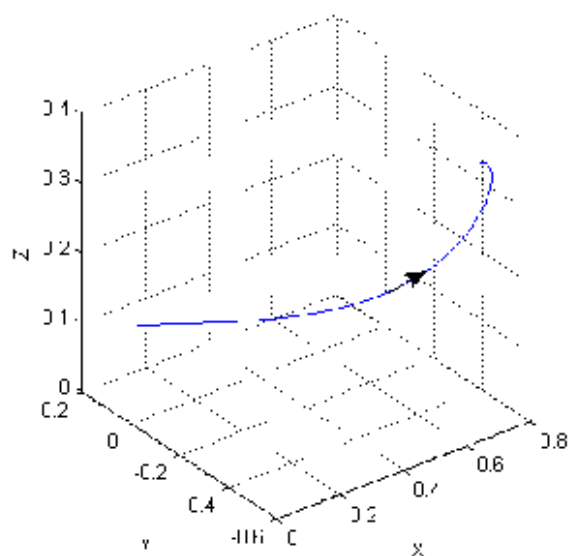

Fig, 5. Chemostat system with cilution control parameter $Z$ Y $Y$ Y $X$ for $k_{1}=100 ; k_{2}=30 ; k_{3}=70$.
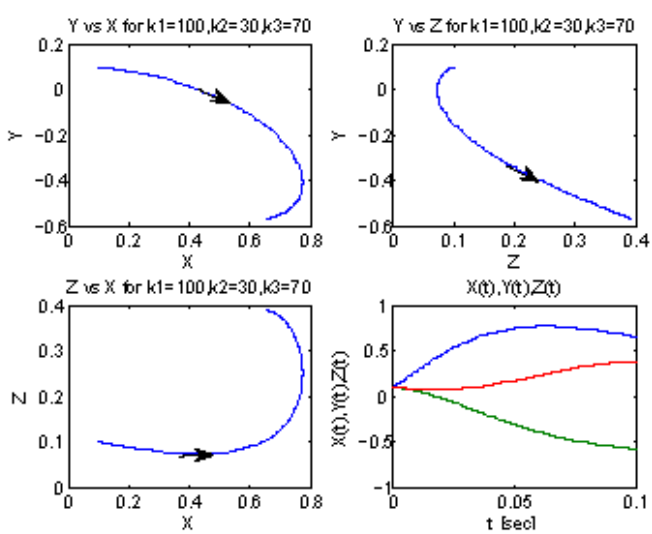

Fig, 6. Chemostat system with dilution control parameter variables phase sposes and time beharior for $k_{1}=100 ; k_{2}=30 ; k_{3}=70$.

Dimensional parameters have the following meaning:

$$
\begin{aligned}
& \psi \rightarrow \frac{w_{0}}{D} ; \gamma \rightarrow \frac{\gamma \beta \mathcal{S}^{(0)} \delta}{D} ; k_{1} \rightarrow k_{1} \delta S^{(0)} \\
& k_{2} \rightarrow k_{2} S(\mathrm{O}) \beta \delta ; k_{3} \rightarrow S(0) k_{3} \\
& S^{(0)} \geq 0 ; B(0)>0 ; \frac{S}{S(0)} \rightarrow S \\
& \stackrel{\circ}{S(0)} \rightarrow a ; f(S)=\frac{V_{\text {mas }} S / S^{(0)}}{\alpha / \mathcal{S}(0)+S / S(0)}
\end{aligned}
$$

(179) Let take the phages differential equation

$S^{(0)}$ term has units of concentration. $D[1 / \mathrm{sec}]$ has units of reciprocal time. We already scale the nutrient equation. The scales muttient equation is:

$$
\frac{d S}{d t}=(1-S)\left(B k_{1}+V k_{2}+S k_{3}\right)-\psi f(S) B
$$

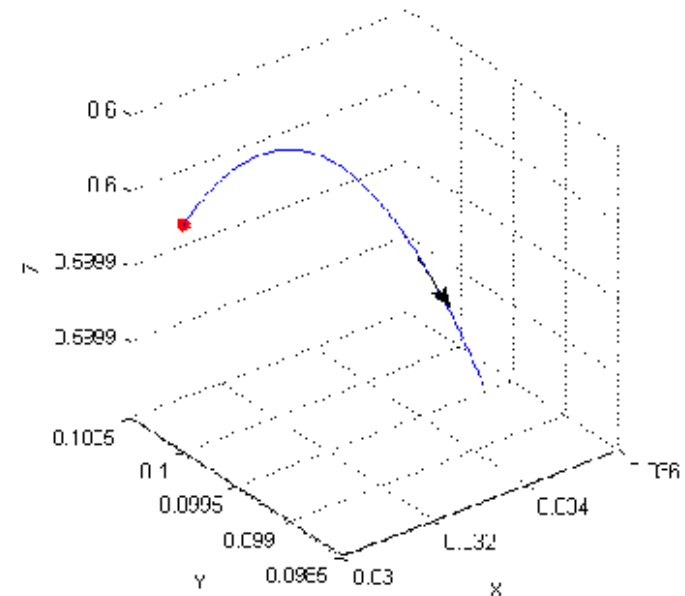

Fiz, 7. Chercostat systern with tilution contol parameter $Z$ Y $Y$ is $X$ for $k_{1}=0.2 ; k_{2}=0.3 ; k_{3}=0.5$.
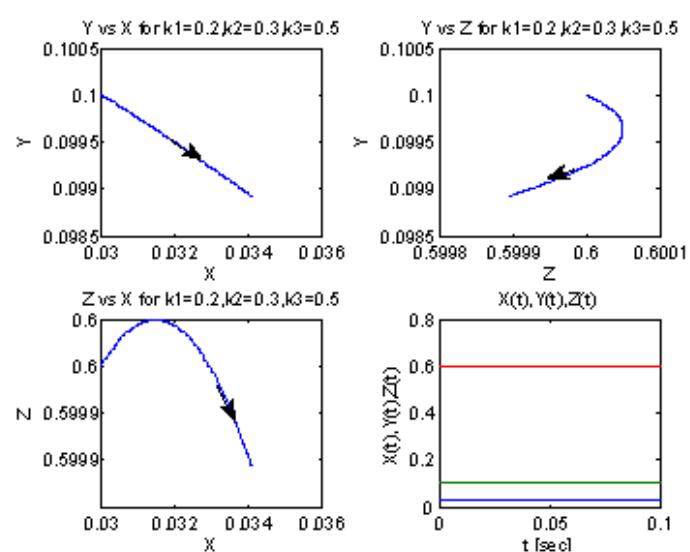

Fig, 8. Chemostat system with tilution control parametor Yariables phase paces ard time behavior for $k_{1}=0.2 ; k_{2}=0.3 ; k_{3}=0.5$.

The scaled bacteria equation:

$$
\frac{d B}{d t}=\psi f(S) B-\gamma V B-B\left[B k_{1}+V k_{2}+S k_{3}\right]
$$

$$
\begin{gathered}
\frac{V}{S(0) \beta \delta} \rightarrow V ; \frac{\varphi\left[\frac{1}{\sec }\right]}{D\left[\frac{1}{\sec }\right]} \rightarrow \varphi ; \frac{B}{S(0) \delta} \rightarrow B \\
\frac{d V}{d t}=-\varphi V-\gamma V B+\beta \gamma V(t-\tau) B(t-\tau)-V(t) D
\end{gathered}
$$




$$
\begin{aligned}
& \begin{array}{l}
\psi \rightarrow \frac{\psi}{D} ; \gamma \rightarrow \frac{\gamma \beta S^{(0)} \delta}{D} ; k_{1} \rightarrow k_{1} \delta S^{(0)} \\
k_{2} \rightarrow k_{2} S^{(0)} \beta \delta ; k_{3} \rightarrow S^{(0)} k_{3}
\end{array} \\
& S^{(0)} \geq 0 ; B(0)>0 ; \frac{S}{S(0)} \rightarrow S ; \frac{a}{S(0)} \rightarrow a \\
& f(S)=\frac{V_{\text {max }} S / S^{(0)}}{a / S^{(0)}+S / S^{(0)}} \\
& D=B k_{1}+V k_{2}+S k_{3} \\
& \frac{d V}{d t}=-\varphi V-\gamma V B+\beta \gamma V(t-\tau) B(t-\tau) \\
& { }_{-V}^{d t}(t)\left[B k_{1}+V k_{2}+S k_{3}\right] \\
& \frac{d}{d t}\left[\frac{V}{S(0) \beta \delta}\right]=-\varphi V \frac{1}{S(0) \beta \delta}-\gamma V \frac{1}{S(0) \beta \delta} B \\
& +\beta \gamma \frac{S^{(0)} \beta \delta}{D} V(t-\tau) \frac{1}{S^{(0)} \beta \delta} B(t-\tau) \frac{1}{S(0)} \frac{D}{\beta} \\
& -V(t) \frac{1}{S^{(0)} \beta \delta}\left[\frac{B}{S^{(0)} \delta} k_{1} S^{(0)} \delta+V \frac{1}{S^{(0)} \beta \delta} k_{2} S^{(0)} \beta \delta\right. \\
& \left.+\frac{S}{S^{(0)}} k_{3} S^{(0)}\right] \\
& \begin{array}{l}
V(t-\tau) \frac{1}{S^{(0)} \beta \delta} \rightarrow V(t-\tau) \\
B(t-\tau) \frac{1}{S^{(0)} \delta} \rightarrow B(t-\tau)
\end{array} \\
& \frac{d}{d t}\left[\frac{V}{S(0) \beta \delta}\right]=-\varphi V \frac{1}{S^{(0)} \beta \delta}-\gamma \frac{S^{(0)} \beta \delta}{D} V \frac{1}{S^{(0)} \beta \delta} B \frac{1}{S^{(0)} \delta} \frac{D}{\beta} \\
& +\beta \gamma \frac{S^{(0)} \beta \delta}{D} V(t-\tau) \frac{1}{S(0) \beta \delta} B(t-\tau) \frac{1}{S(0) \delta} \frac{D}{\beta} \\
& -V(t) \frac{1}{S(0) \beta \delta}\left[\frac{B}{S^{(0)} \delta} k_{1} S^{(0)} \delta+V \frac{1}{S^{(0)} \beta \delta} k_{2} S^{(0)} \beta \delta\right. \\
& \left.+\frac{S}{S(0)} k_{3} S^{(0)}\right] \\
& \frac{d}{d t} V=-\varphi V-\gamma V B \frac{D}{\beta}+\beta \gamma V(t-\tau) B(t-\tau) \frac{D}{\beta} \\
& -V(t)\left[B k_{1}+V k_{2}+S k_{3}\right]
\end{aligned}
$$

Measuring $S, a, B / \delta$ in units of $S^{(0)}$, and time in units of $D^{-1}$

$$
\begin{aligned}
& \frac{d}{d t} V \frac{1}{D}=-\varphi \frac{1}{D} V-\gamma V B \frac{1}{\beta} \\
& +\gamma V(t-\tau) B(t-\tau) \\
& -V(t)\left[B k_{1}+V k_{2}+S k_{3}\right] \frac{1}{D} \\
& \frac{d}{d t} V \frac{1}{D} \rightarrow \frac{d}{d t} V \\
& {\left[B k_{1}+V k_{2}+S k_{3}\right] \frac{1}{D} \rightarrow\left[B k_{1}+V k_{2}+S k_{3}\right]} \\
& \frac{B\left[\frac{1}{m l}\right]}{S(0)\left[\frac{1}{m m^{\prime}}\right] \delta} k_{1}\left[\frac{m l}{\sec }\right] \delta S^{(0)}\left[\frac{1}{m l}\right] \rightarrow\left[\frac{1}{\mathrm{sec}}\right]
\end{aligned}
$$

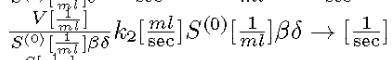

$$
\begin{aligned}
& \frac{S\left[\frac{1}{m l}\right]}{S(0)\left[\frac{1}{m b l}\right]} S^{(0)}\left[\frac{1}{m l}\right] k_{3}\left[\frac{m l}{\sec }\right] \rightarrow\left[\frac{1}{\sec }\right] \\
& \frac{\varphi\left[\frac{1}{\sec _{e}}\right]}{D\left[\frac{1}{\sec }\right]} \rightarrow \varphi \Rightarrow \frac{d}{d t} V=-\varphi V-\gamma V B \frac{1}{\beta} \\
& +\gamma V(t-\tau) B(t-\tau)-V(t)\left[B k_{1}+V k_{2}+S k_{3}\right]
\end{aligned}
$$

The scaled phage equation:

$$
\begin{aligned}
& \frac{d}{d t} V=-\varphi V-\gamma V B \frac{1}{\beta}+\gamma V(t-\tau) B(t-\tau) \\
& -V(t)\left[B k_{1}+V k_{2}+S k_{3}\right]
\end{aligned}
$$

The Chemostat (One compartment Gradostat) with delay variables and control parameter scaled equations:

$$
\begin{aligned}
& \frac{d S}{d t}=(1-S)\left(B k_{1}+V k_{2}+S k_{3}\right)-\psi f(S) B \\
& \frac{d}{d t} V=-\varphi V-\gamma V B \frac{1}{\beta}+\gamma V(t-\tau) B(t-\tau) \\
& -V(t)\left[B k_{1}+V k_{2}+S k_{3}\right] \\
& \frac{d B}{d t}=\psi f(S) B-\gamma V B-B\left[B k_{1}+V k_{2}+S k_{3}\right]
\end{aligned}
$$

Stability analysis: The standard local stability analysis about any one of the equilibrium points of Chemostat system with control variables consists in adding to its coordinates $[V B S]$ arbitrarily small increments of exponential form [vbs] $e^{\lambda t}$, and retaining the first order terms in $v, b, s$. The system of three homogeneous equations leads to a polynomial characteristics equation in the eigenvalues $\lambda$. The polynomial characteristics equations accept by set the below phage, bacteria, and nutrient equations (delayed and un-delayed) into three Chemostat scaled equations. The phages, bacteria, and nutrient fixed values with arbitrarily small increments of exponential form $[v \mathrm{bs}] e^{\lambda t}$ are: $i=0$ (first fixed point), $i=1$ (second fixed point), $i=2$ (third fixed point) [2] [3].

$$
\begin{aligned}
& V(t)=V^{(i)}+v e^{\lambda t} \\
& B(t)=B^{(i)}+b e^{\lambda t} ; S(t)=S^{(i)}+s e^{\lambda t} \\
& V(t-\tau)=V^{(i)}+v e^{\lambda(t-\tau)} \\
& B(t-\tau)=B^{(i)}+b e^{\lambda(t-\tau)} \\
& \forall \mathrm{i}=0,1,2
\end{aligned}
$$

We choose the above expressions for our $V(t), B(t), S(t)$ as small displacement $[v b s]$ from the system fixed points at time $t=0$.

$$
\begin{aligned}
& V(t=0)=V^{(i)}+v ; B(t=0)=B^{(i)}+b \\
& S(t=0)=S^{(i)}+s
\end{aligned}
$$

for $\lambda<0, t>0$ the selected fixed point is stable otherwise $\lambda>0, t>0$ is Unstable. Our system tends to the selected fixed point exponentially for $\lambda<0, t>0$ otherwise go away from the selected fixed point exponentially. $\lambda$ is the eigenvalue parameter which establish if the fixed point is stable or Unstable, additionally his absolute value $(|\lambda|)$ establish the speed of flow toward or away from the selected fixed point [10] [11].

We already approve the equation for $f(S)$ :

$$
f(S)=\frac{\left[S^{(i)}+s e^{\lambda t}\right] a+\left[S^{(i)}\right]^{2}}{\left[a+S^{(i)}\right]^{2}}
$$

First we take the Chemostat Nutrient differential equation

$$
\frac{d S}{d t}=(1-S)\left(B k_{1}+V k_{2}+S k_{3}\right)-\psi f(S) B
$$

Adding to its coordinates $[V B S]$ arbitrarily small increments of exponential form $[v \mathrm{bs}] e^{\lambda t}$, and retaining the first order terms in $v, b, s$. 


$$
\begin{aligned}
& S(t)=S^{(i)}+s e^{\lambda t} ; B(t)=B^{(i)}+b e^{\lambda t} \\
& \frac{d S(t)}{d t}=s \lambda e^{\lambda t} ; V(t)=V^{(i)}+v e^{\lambda t} \\
& \frac{d V(t)}{d t}=v \lambda e^{\lambda t} \\
& s \lambda e^{\lambda t}=\left(1-\left[S^{(i)}+s e^{\lambda t}\right]\right)\left(\left[B^{(i)}+b e^{\lambda t}\right] k_{1}\right. \\
& \left.+\left[V^{(i)}+v e^{\lambda t}\right] k_{2}+\left[S^{(i)}+s e^{\lambda t}\right] k_{3}\right) \\
& -\psi\left\{\frac{\left[S^{(i)}+s e^{\lambda t}\right] a+\left[S^{(i)}\right]^{2}}{\left[a+S^{(i)}\right]^{2}}\right\}\left[B^{(i)}+b e^{\lambda t}\right] \\
& s \lambda e^{\lambda t}=\left(\left[1-S^{(i)}\right]-s e^{\lambda t}\right)\left(B^{(i)} k_{1}+b k_{1} e^{\lambda t}\right. \\
& \left.+V^{(i)} k_{2}+v k_{2} e^{\lambda t}+S^{(i)} k_{3}+s k_{3} e^{\lambda t}\right) \\
& -\psi\left\{\frac{S^{(i)} a+s a e^{\lambda t}+\left[S^{(i)}\right]^{2}}{\left[a+S^{(i)}\right]^{2}}\right\}\left[B^{(i)}+b e^{\lambda t}\right] \\
& s \lambda e^{\lambda t}=\left(\left[1-S^{(i)}\right]-s e^{\lambda t}\right)\left(B^{(i)} k_{1}+b k_{1} e^{\lambda t}\right. \\
& \left.+V^{(i)} k_{2}+v k_{2} e^{\lambda t}+S^{(i)} k_{3}+s k_{3} e^{\lambda t}\right) \\
& -\left\{\psi \frac{S^{(i)} a B^{(i)}}{\left[a+S^{(i)}\right]^{2}}+\psi \frac{S^{(i)} a b e^{\lambda t}}{\left[a+S^{(i)}\right]^{2}}+\psi \frac{s a e^{\lambda t} B^{(i)}}{\left[a+S^{(i)}\right]^{2}}\right. \\
& \left.+\psi \frac{s a e^{\lambda t} b e^{\lambda t}}{\left[a+S^{(i)}\right]^{2}}+\psi \frac{\left[S^{(i)}\right]^{2} B^{(i)}}{\left[a+S^{(i)}\right]^{2}}+\psi \frac{\left[S^{(i)}\right]^{2} b e^{\lambda t}}{\left[a+S^{(i)}\right]^{2}}\right\} \\
& s \lambda e^{\lambda t}=\left(\left[1-S^{(i)}\right]-s e^{\lambda t}\right)\left(B^{(i)} k_{1}+b k_{1} e^{\lambda t}\right. \\
& \left.+V^{(i)} k_{2}+v k_{2} e^{\lambda t}+S^{(i)} k_{3}+s k_{3} e^{\lambda t}\right) \\
& -\left\{\psi \frac{S^{(i)} a B^{(i)}}{\left[a+S^{(i)}\right]^{2}}+\psi \frac{S^{(i)} a b e^{\lambda t}}{\left[a+S^{(i)}\right]^{2}}+\psi \frac{s a e^{\lambda t} B^{(i)}}{\left[a+S^{(i)}\right]^{2}}\right. \\
& \left.+\psi \frac{s a e^{\lambda t} b e^{\lambda t}}{\left[a+S^{(i)}\right]^{2}}+\psi \frac{\left[S^{(i)}\right]^{2} B^{(i)}}{\left[a+S^{(i)}\right]^{2}}+\psi \frac{\left[S^{(i)}\right]^{2} b e^{\lambda t}}{\left[a+S^{(i)}\right]^{2}}\right\} \\
& s \lambda e^{\lambda t}=\left[1-S^{(i)}\right]\left[B^{(i)} k_{1}+V^{(i)} k_{2}+S^{(i)} k_{3}\right] \\
& +\left[1-S^{(i)}\right]\left[b k_{1} e^{\lambda t}+v k_{2} e^{\lambda t}+s k_{3} e^{\lambda t}\right] \\
& -\left[B^{(i)} k_{1}+V^{(i)} k_{2}+S^{(i)} k_{3}\right] s e^{\lambda t} \\
& -\left[b k_{1} e^{\lambda t}+v k_{2} e^{\lambda t}+s k_{3} e^{\lambda t}\right] s e^{\lambda t} \\
& -\left\{\psi \frac{S^{(i)} a B^{(i)}}{\left[a+S^{(i)}\right]^{2}}+\psi \frac{S^{(i)} a b e^{\lambda t}}{\left[a+S^{(i)}\right]^{2}}+\psi \frac{s a e^{\lambda t} B^{(i)}}{\left[a+S^{(i)}\right]^{2}}\right. \\
& \left.+\psi \frac{s a e^{\lambda t} b e^{\lambda t}}{\left[a+S^{(i)}\right]^{2}}+\psi \frac{\left[S^{(i)}\right]^{2} B^{(i)}}{\left[a+S^{(i)}\right]^{2}}+\psi \frac{\left[S^{(i)}\right]^{2} b e^{\lambda t}}{\left[a+S^{(i)}\right]^{2}}\right\}
\end{aligned}
$$

$s b \approx 0 ; v s \approx 0 ; s^{2} \approx 0$ and the equation get the below expression :

$$
\begin{aligned}
& s \lambda e^{\lambda t}=\left[1-S^{(i)}\right]\left[B^{(i)} k_{1}+V^{(i)} k_{2}+S^{(i)} k_{3}\right] \\
& +\left[1-S^{(i)}\right]\left[b k_{1} e^{\lambda t}+v k_{2} e^{\lambda t}+s k_{3} e^{\lambda t}\right] \\
& -\left[B^{(i)} k_{1}+V^{(i)} k_{2}+S^{(i)} k_{3}\right] s e^{\lambda t} \\
& -\left\{\psi \frac{S^{(i)} a B^{(i)}}{\left[a+S^{(i)}\right)}+\psi \frac{S^{(i)} a b e^{\lambda t}}{\left[a+S^{(i)}\right]^{2}}+\psi \frac{s a e^{\lambda t} B^{(i)}}{\left[a+S^{(i)}\right]^{2}}\right. \\
& \left.+\psi \frac{\left[S^{(i)}\right]^{2} B^{(i)}}{\left[a+S^{(i)}\right]^{2}}+\psi \frac{\left[S^{(i)}\right]^{2} b e^{\lambda t}}{\left[a+S^{(i)}\right]^{2}}\right\}
\end{aligned}
$$

Any Chemostat fixed point:

$$
\begin{aligned}
\frac{d S}{d t}= & ; f\left(S^{(i)}\right)=\left.\frac{V_{M} S^{(i)}}{a+S^{(i)}}\right|_{V_{M}=1}=\frac{S^{(i)}}{a+S^{(i)}} \\
& \left(1-S^{(i)}\right)\left(B^{(i)} k_{1}+V^{(i)} k_{2}+S^{(i)} k_{3}\right) \\
& -\psi f\left(S^{(i)}\right) B^{(i)}=0 \\
& \left(1-S^{(i)}\right)\left(B^{(i)} k_{1}+V^{(i)} k_{2}+S^{(i)} k_{3}\right) \\
& -\psi \frac{V_{M} S^{(i)}}{a+S^{(i)}} B^{(i)}=0
\end{aligned}
$$

$$
\begin{aligned}
& s \lambda e^{\lambda t}=\left[1-S^{(i)}\right]\left[B^{(i)} k_{1}+V^{(i)} k_{2}+S^{(i)} k_{3}\right] \\
& +\left[1-S^{(i)}\right]\left[b k_{1} e^{\lambda t}+v k_{2} e^{\lambda t}+s k_{3} e^{\lambda t}\right] \\
& -\left[B^{(i)} k_{1}+V^{(i)} k_{2}+S^{(i)} k_{3}\right] s e^{\lambda t} \\
& -\left\{\psi \frac{S^{(i)} a B^{(i)}}{\left[a+S^{(i)}\right]^{2}}+\psi \frac{\left[S^{(i)}\right]^{2} B^{(i)}}{\left[a+S^{(i)}\right]^{2}}+\psi \frac{S^{(i)} a b e^{\lambda t}}{\left[a+S^{(i)}\right]^{2}}\right. \\
& \left.+\psi \frac{s a e^{\lambda t} B^{(i)}}{\left[a+S^{(i)}\right]^{2}}+\psi \frac{\left[S^{(i)}\right]^{2} b e^{\lambda t}}{\left[a+S^{(i)}\right]^{2}}\right\} \\
& \psi \\
& \psi \frac{S^{(i)} a B^{(i)}}{\left[a+S^{(i)}\right]^{2}}+\psi \frac{\left[S^{(i)}\right]^{2} B^{(i)}}{\left[a+S^{(i)}\right]^{2}} \\
& =\psi \frac{B^{(i)} S^{(i)}}{\left(a+S^{(i)}\right)}=\psi B^{(i)} f\left(S^{(i)}\right)
\end{aligned}
$$

$s \lambda e^{\lambda t}=\left[1-S^{(i)}\right]\left[B^{(i)} k_{1}+V^{(i)} k_{2}+S^{(i)} k_{3}\right]$

$-\psi \frac{B^{(i)} S^{(i)}}{\left(a+S^{(i)}\right)}+\left[1-S^{(i)}\right]\left[b k_{1} e^{\lambda t}+v k_{2} e^{\lambda t}+s k_{3} e^{\lambda t}\right]$

$-\left[B^{(i)} k_{1}+V^{(i)} k_{2}+S^{(i)} k_{3}\right] s e^{\lambda t}$

$-\psi \frac{S^{(i)} a b e^{\lambda t}}{\left.\mid a+S^{(i)}\right]^{2}}-\psi \frac{s a e^{\lambda t} B^{(i)}}{\left[a+S^{(i)}\right]^{2}}-\psi \frac{\left[S^{(i)}\right]^{2} b e^{\lambda t}}{\left[a+S^{(i)}\right]^{2}}$

$$
\begin{aligned}
& s \lambda e^{\lambda t}=\left[1-S^{(i)}\right]\left[B^{(i)} k_{1}+V^{(i)} k_{2}+S^{(i)} k_{3}\right] \\
& -\psi B^{(i)} f\left(S^{(i)}\right)+\left[1-S^{(i)}\right]\left[b k_{1} e^{\lambda t}+v k_{2} e^{\lambda t}+s k_{3} e^{\lambda t}\right] \\
& -\left[B^{(i)} k_{1}+V^{(i)} k_{2}+S^{(i)} k_{3}\right] s e^{\lambda t}-\psi \frac{S^{(i)} a b e^{\lambda t}}{\left[a+S^{(i)}\right]^{2}} \\
& -\psi \frac{s a e^{\lambda t} B^{(i)}}{\left[a+S^{(i)}\right]^{2}}-\psi \frac{\left[S^{(i)}\right]^{2} b e^{\lambda t}}{\left[a+S^{(i)}\right]^{2}} \\
& \quad\left(1-S^{(i)}\right)\left(B^{(i)} k_{1}+V^{(i)} k_{2}+S^{(i)} k_{3}\right) \\
& -\psi f\left(S^{(i)}\right) B^{(i)}=0
\end{aligned}
$$

Divide above equation by $e^{\lambda \epsilon}$ and we get

$$
\begin{aligned}
& {\left[1-S^{(i)}\right]\left[b k_{1}+v k_{2}+s k_{3}\right]} \\
& -\left[B^{(i)} k_{1}+V^{(i)} k_{2}+S^{(i)} k_{3}\right] s \\
& -\psi \frac{S^{(i)} a b}{\left[a+S^{(i)}\right]^{2}} \\
& -\psi \frac{s a B^{(i)}}{\left[a+S^{(i)}\right]^{2}}-\psi \frac{\left[S^{(i)}\right]^{2} b}{\left[a+S^{(i)}\right]^{2}}-s \lambda=0
\end{aligned}
$$

$s\left\{\left[1-S^{(i)}\right] k_{3}-\left[B^{(i)} k_{1}+V^{(i)} k_{2}+S^{(i)} k_{3}\right]\right.$

$$
\left.-\psi \frac{a B^{(i)}}{\left[a+S^{(i)}\right]^{2}}-\lambda\right\}+v\left[1-S^{(i)}\right] k_{2}+b\left\{\left[1-S^{(i)}\right] k_{1}\right.
$$$$
\left.-\psi \frac{S^{(i)} a}{\left[a+S^{(i)}\right]^{2}}-\psi \frac{\left[S^{(i)}\right]^{2}}{\left[a+S^{(i)}\right]^{2}}\right\}=0
$$

We take the Chemostat Phage differential equation:

$$
\begin{aligned}
& s \lambda e^{\lambda t}=\left[1-S^{(i)}\right]\left[b k_{1} e^{\lambda t}+v k_{2} e^{\lambda t}+s k_{3} e^{\lambda t}\right] \\
& -\left[B^{(i)} k_{1}+V^{(i)} k_{2}+S^{(i)} k_{3}\right] s e^{\lambda t} \\
& -\psi \frac{S^{(i)} a b e^{\lambda t}}{\left[a+S^{(i)}\right]^{2}}-\psi \frac{s a e^{\lambda t} B^{(i)}}{\left[a+S^{(i)}\right]^{2}}-\psi \frac{\left[S^{(i)}\right]^{2} b e^{\lambda t}}{\left[a+S^{(i)}\right]^{2}} \\
& {\left[1-S^{(i)}\right]\left[b k_{1} e^{\lambda t}+v k_{2} e^{\lambda t}+s k_{3} e^{\lambda t}\right]} \\
& -\left[B^{(i)} k_{1}+V^{(i)} k_{2}+S^{(i)} k_{3}\right] s e^{\lambda t}-\psi \frac{S^{(i)} a b e^{\lambda t}}{\left[a+S^{(i)}\right]^{2}} \\
& -\psi \frac{s a e^{\lambda t} B^{(i)}}{\left[a+S^{(i)}\right]^{2}}-\psi \frac{\left[S^{(i)}\right]^{2} b e^{\lambda t}}{\left[a+S^{(i)}\right]^{2}}-s \lambda e^{\lambda t}=0 \\
& \frac{d}{d t} V=-\varphi V-\gamma V B \frac{1}{\beta}+\gamma V(t-\tau) B(t-\tau) \\
& -V(t)\left[B k_{1}+V k_{2}+S k_{3}\right]
\end{aligned}
$$

Adding to its coordinates $[V B S]$ arbitrarily small increments of exponential form $[v \mathrm{bs}] e^{\lambda t}$, and retaining the first order

terms in $v, b, s$. 
Ofer Aluf; Dynamic of Bacterial and Phage Populations Maintained in Chemostat System with Dilution Control Parameter, Transactions on Networks and Communications, Volume 4 No. 3, June (2016); pp: 1-21

$$
\begin{aligned}
& S(t)=S^{(i)}+s e^{\lambda t} ; B(t)=B^{(i)}+b e^{\lambda t} \\
& \frac{d S(t)}{d t}=s \lambda e^{\lambda t} ; V(t)=V^{(i)}+v e^{\lambda t} \\
& \frac{d V(t)}{d t}=v \lambda e^{\lambda t} \\
& V(t-\tau)=V^{(i)}+v e^{\lambda(t-\tau)}=V^{(i)}+v e^{\lambda t} e^{-\lambda \tau} \\
& B(t-\tau)=B^{(i)}+b e^{\lambda(t-\tau)}=B^{(i)}+b e^{\lambda t} e^{-\lambda \tau} \\
& v \lambda e^{\lambda t}=-\varphi\left[V^{(i)}+v e^{\lambda t}\right] \\
& -\gamma\left[V^{(i)}+v e^{\lambda t}\right]\left[B^{(i)}+b e^{\lambda t}\right] \\
& +\gamma\left[V^{(i)}+v e^{\lambda t} e^{-\lambda \tau}\right]\left[B^{(i)}+b e^{\lambda t} e^{-\lambda \tau}\right] \\
& -\left[V^{(i)}+v e^{\lambda t}\right]\left\{\left[B^{(i)}+b e^{\lambda t}\right] k_{1}\right. \\
& \left.+\left[V^{(i)}+v e^{\lambda t}\right] k_{2}+\left[S^{(i)}+s e^{\lambda t}\right] k_{3}\right\} \\
& v \lambda e^{\lambda t}=-\varphi V^{(i)}-\varphi v e^{\lambda t}-\gamma V^{(i)} B^{(i)} \frac{1}{\beta} \\
& -\gamma V^{(i)} b e^{\lambda t} \frac{1}{\beta}-\gamma v e^{\lambda t} B^{(i)} \frac{1}{\beta}-\gamma v b e^{\lambda t} e^{\lambda t} \frac{1}{\beta} \\
& +\gamma V^{(i)} B^{(i)}+\gamma V^{(i)} b e^{\lambda t} e^{-\lambda \tau}+\gamma v e^{\lambda t} e^{-\lambda \tau} B^{(i)} \\
& +\gamma v b e^{\lambda t} e^{-\lambda \tau} e^{\lambda t} e^{-\lambda \tau}-\left[V^{(i)}+v e^{\lambda t}\right]\left\{B^{(i)} k_{1}\right. \\
& \left.+b k_{1} e^{\lambda t}+V^{(i)} k_{2}+v k_{2} e^{\lambda t}+S^{(i)} k_{3}+s k_{3} e^{\lambda t}\right\} \\
& v \lambda e^{\lambda t}=-\varphi V^{(i)}-\varphi v e^{\lambda t}-\gamma V^{(i)} B^{(i)} \frac{1}{\beta} \\
& -\gamma V^{(i)} b e^{\lambda t} \frac{1}{\beta}-\gamma v e^{\lambda t} B^{(i)} \frac{1}{\beta}-\gamma v b e^{\lambda t} e^{\lambda t} \frac{1}{\beta} \\
& +\gamma V^{(i)} B^{(i)}+\gamma V^{(i)} b e^{\lambda t} e^{-\lambda \tau}+\gamma v e^{\lambda t} e^{-\lambda \tau} B^{(i)} \\
& +\gamma v b e^{\lambda t} e^{-\lambda \tau} e^{\lambda t} e^{-\lambda \tau}-\left[V^{(i)}+v e^{\lambda t}\right]\left\{B^{(i)} k_{1}\right. \\
& \left.+b k_{1} e^{\lambda t}+V^{(i)} k_{2}+v k_{2} e^{\lambda t}+S^{(i)} k_{3}+s k_{3} e^{\lambda t}\right\} \\
& v \lambda e^{\lambda t}=-\varphi V^{(i)}-\varphi v e^{\lambda t}-\gamma V^{(i)} B^{(i)} \frac{1}{\beta} \\
& -\gamma V^{(i)} b e^{\lambda t} \frac{1}{\beta}-\gamma v e^{\lambda t} B^{(i)} \frac{1}{\beta}-\gamma v b e^{\lambda t} e^{\lambda t} \frac{1}{\beta} \\
& +\gamma V^{(i)} B^{(i)}+\gamma V^{(i)} b e^{\lambda t} e^{-\lambda \tau}+\gamma v e^{\lambda t} e^{-\lambda \tau} B^{(i)} \\
& +\gamma v b e^{\lambda t} e^{-\lambda \tau} e^{\lambda t} e^{-\lambda \tau}-V^{(i)}\left[B^{(i)} k_{1}+V^{(i)} k_{2}\right. \\
& \left.+S^{(i)} k_{3}\right]-V^{(i)}\left[b k_{1}+v k_{2}+s k_{3}\right] e^{\lambda t}-\left[B^{(i)} k_{1}\right. \\
& \left.+V^{(i)} k_{2}+S^{(i)} k_{3}\right] v e^{\lambda t}-\left[b k_{1}+v k_{2}\right. \\
& +s k_{3} e^{\lambda t} v e^{\lambda t} \\
& v \lambda e^{\lambda t}=-\varphi V^{(i)}-\gamma V^{(i)} B^{(i)} \frac{1}{\beta}+\gamma V^{(i)} B^{(i)} \\
& -V^{(i)}\left[B^{(i)} k_{1}+V^{(i)} k_{2}+S^{(i)} k_{3}\right]-\varphi v e^{\lambda t} \\
& -\gamma V^{(i)} b e^{\lambda t} \frac{1}{\beta}-\gamma v e^{\lambda t} B^{(i)} \frac{1}{\beta}-\gamma v b e^{\lambda t} e^{\lambda t} \frac{1}{\beta} \\
& +\gamma V^{(i)} b e^{\lambda t} e^{-\lambda \tau}+\gamma v e^{\lambda t} e^{-\lambda \tau} B^{(i)} \\
& +\gamma v b e^{\lambda t} e^{-\lambda \tau} e^{\lambda t} e^{-\lambda \tau}-V^{(i)}\left[b k_{1}+v k_{2}+s k_{3}\right] e^{\lambda t} \\
& -\left[B^{(i)} k_{1}+V^{(i)} k_{2}+S^{(i)} k_{3}\right] v e^{\lambda t} \\
& -\left[b k_{1}+v k_{2}+s k_{3}\right] e^{\lambda t} v e^{\lambda t}
\end{aligned}
$$

Any Chemostat fixed point:

$$
\lim _{t \rightarrow \infty} V(t-\tau)=\lim _{t \rightarrow \infty} V(t) ; \lim _{t \rightarrow \infty} B(t-\tau)=\lim _{t \rightarrow \infty} B(t)
$$

$$
\begin{aligned}
& \frac{d}{d t} V=0 \Rightarrow-\varphi V^{(i)}-\gamma V^{(i)} B^{(i)} \frac{1}{\beta}+\gamma V^{(i)} B^{(i)} \\
& -V^{(i)}\left[B^{(i)} k_{1}+V^{(i)} k_{2}+S^{(i)} k_{3}\right]=0 \\
& v \lambda e^{\lambda t}=-\varphi v e^{\lambda t}-\gamma V^{(i)} b e^{\lambda t} \frac{1}{\beta}-\gamma v e^{\lambda t} B^{(i)} \frac{1}{\beta} \\
& -\gamma v b e^{\lambda t} e^{\lambda t} \frac{1}{\beta}+\gamma V^{(i)} b e^{\lambda t} e^{-\lambda \tau}+\gamma v e^{\lambda t} e^{-\lambda \tau} B^{(i)} \\
& +\gamma v b e^{\lambda t} e^{-\lambda \tau} e^{\lambda t} e^{-\lambda \tau}-V^{(i)}\left[b k_{1}+v k_{2}+s k_{3}\right] e^{\lambda t} \\
& -\left[B^{(i)} k_{1}+V^{(i)} k_{2}+S^{(i)} k_{3}\right] v e^{\lambda t} \\
& -\left[b v k_{1}+v v k_{2}+s v k_{3}\right] e^{2 \lambda t}
\end{aligned}
$$

$$
v b \approx 0 ; s v \approx 0 ; v^{2} \approx 0
$$

$$
\begin{aligned}
& v \lambda e^{\lambda t}=-\varphi v e^{\lambda t}-\gamma V^{(i)} b e^{\lambda t} \frac{1}{\beta}-\gamma v e^{\lambda t} B^{(i)} \frac{1}{\beta} \\
& +\gamma V^{(i)} b e^{\lambda t} e^{-\lambda \tau}+\gamma v e^{\lambda t} e^{-\lambda \tau} B^{(i)} \\
& -V^{(i)}\left[b k_{1}+v k_{2}+s k_{3}\right] e^{\lambda t}-\left[B^{(i)} k_{1}+V^{(i)} k_{2}\right. \\
& \left.+S^{(i)} k_{3}\right] v e^{\lambda t} \\
& -\varphi v e^{\lambda t}-\gamma V^{(i)} b e^{\lambda t} \frac{1}{\beta}-\gamma v e^{\lambda t} B^{(i)} \frac{1}{\beta} \\
& +\gamma V^{(i)} b e^{\lambda t} e^{-\lambda \tau}+\gamma \eta e^{\lambda t} e^{-\lambda \tau} B^{(i)} \\
& -V^{(i)}\left[b k_{1}+v k_{2}+s k_{3}\right] e^{\lambda t}-\left[B^{(i)} k_{1}+V^{(i)} k_{2}+\right. \\
& \left.S^{(i)} k_{3}\right] v e^{\lambda t}-v \lambda e^{\lambda t}=0 \\
& \quad-s V^{(i)} k_{3}{ }^{\lambda t}+v\left\{\gamma e^{\lambda t} e^{-\lambda \tau} B^{(i)}-\varphi e^{\lambda t}\right. \\
& \quad-\gamma e^{\lambda t} B^{(i)} \frac{1}{\beta}-\lambda e^{\lambda t}-V^{(i)} k_{2} e^{\lambda t} \\
& \left.-\left[B^{(i)} k_{1}+V^{(i)} k_{2}+S^{(i)} k_{3}\right] e^{\lambda t}\right\} \\
& +b\left\{\gamma V^{(i)} e^{\lambda t} e^{-\lambda \tau}-\gamma V^{(i)} e^{\lambda t} \frac{1}{\beta}\right. \\
& \\
& \left.-V^{(i)} k_{1} e^{\lambda t}\right\}=0
\end{aligned}
$$

Divide above equation by $e^{\lambda t}$ and we get

$$
\begin{aligned}
& -s V^{(i)} k_{3}+v\left\{\gamma e^{-\lambda \tau} B^{(i)}-\varphi-\gamma B^{(i)} \frac{1}{\beta}\right. \\
& \left.-V^{(i)} k_{2}-\left[B^{(i)} k_{1}+V^{(i)} k_{2}+S^{(i)} k_{3}\right]-\lambda\right\} \\
& +b\left\{\gamma V^{(i)} e^{-\lambda \tau}-\gamma V^{(i)} \frac{1}{\beta}-V^{(i)} k_{1}\right\}=0
\end{aligned}
$$

We take the Chemostat bacteria differential equation:

$$
\frac{d B}{d t}=\psi f(S) B-\gamma V B-B\left[B k_{1}+V k_{2}+S k_{3}\right]
$$

Adding to its coordinates $[V B S]$ arbitrarily small increments of exponential form $[v \mathrm{bs}] e^{\lambda t}$, and retaining the first order terms in $v, b, s$.

$$
\begin{aligned}
& B(t)=B^{(i)}+b e^{\lambda t} ; \frac{d B(t)}{d t}=b \lambda e^{\lambda t} \\
& \frac{d S(t)}{d t}=s \lambda e^{\lambda t} ; V(t)=V^{(i)}+v e^{\lambda t} \\
& \frac{d V(t)}{d t}=v \lambda e^{\lambda t} ; S(t)=S^{(i)}+s e^{\lambda t}
\end{aligned}
$$

$V(t-\tau)=V^{(i)}+v e^{\lambda(t-\tau)}=V^{(i)}+v e^{\lambda t} e^{-\lambda \tau}$ $B(t-\tau)=B^{(i)}+b e^{\lambda(t-\tau)}=B^{(i)}+b e^{\lambda t} e^{-\lambda \tau}$

$$
\begin{aligned}
& f(S)=\frac{\left[S^{(i)}+s e^{\lambda t}\right] a+\left[S^{(i)}\right]^{2}}{\left.\left[a+S^{(i)}\right)\right]^{2}} \\
& \left.f\left(S^{(i)}\right)\right|_{V_{M}=1}=\frac{S^{(i)}}{\left(a+S^{(i)}\right)}
\end{aligned}
$$

$b \lambda e^{\lambda t}=\psi\left[\frac{\left[S^{(i)}+s e^{\lambda t}\right] a+\left[S^{(i)}\right]^{2}}{\left[a+S^{(i)}\right]^{2}}\right]\left[B^{(i)}+b e^{\lambda t}\right]$ $-\gamma\left[V^{(i)}+v e^{\lambda t}\right]\left[B^{(i)}+b e^{\lambda t}\right]-\left[B^{(i)}+b e^{\lambda t}\right]\left[\left(B^{(i)}\right.\right.$ $\left.\left.+b e^{\lambda t}\right) k_{1}+\left(V^{(i)}+v e^{\lambda t}\right) k_{2}+\left(S^{(i)}+s e^{\lambda t}\right) k_{3}\right]$

$$
\begin{aligned}
& b \lambda e^{\lambda t}=\psi\left[\frac{S^{(i)}}{\left(a+S^{(i)}\right)}+\frac{s a e^{\lambda t}}{\left[a+S^{(i)}\right]^{2}}\right]\left[B^{(i)}+b e^{\lambda t}\right] \\
& -\gamma\left[V^{(i)} B^{(i)}+V^{(i)} b e^{\lambda t}+B^{(i)} v e^{\lambda t}\right. \\
& \left.+v b e^{2 \lambda t}\right]-\left[B^{(i)}+b e^{\lambda t}\right]\left[\left(B^{(i)} k_{1}+V^{(i)} k_{2}\right.\right. \\
& \left.\left.+S^{(i)} k_{3}\right)+\left(b k_{1}+v k_{2}+s k_{3}\right) e^{\lambda t}\right]
\end{aligned}
$$

6)

8)


$b \lambda e^{\lambda t}=\psi f\left(S^{(i)}\right) B^{(i)}+\psi f\left(S^{(i)}\right) b e^{\lambda t}$

$+\psi B^{(i)} \frac{s a e^{\lambda t}}{\left[a+S^{(i)}\right]^{2}}+\psi \frac{s a e^{\lambda t} b e^{\lambda t}}{\left[a+S^{(i)}\right]^{2}}$

$-\gamma\left[V^{(i)} B^{(i)}+V^{(i)} b e^{\lambda t}+B^{(i)} v e^{\lambda t}+v b e^{2 \lambda t}\right]$

$-B^{(i)}\left(B^{(i)} k_{1}+V^{(i)} k_{2}+S^{(i)} k_{3}\right)$

$-B^{(i)}\left(b k_{1}+v k_{2}+s k_{3}\right) e^{\lambda t}-\left(B^{(i)} k_{1}+V^{(i)} k_{2}\right.$

$\left.+S^{(i)} k_{3}\right) b e^{\lambda t}-\left(b k_{1}+v k_{2}+s k_{3}\right) e^{\lambda t} b e^{\lambda t}$

$v s \approx 0 ; v b \approx 0 ; s b \approx 0 ; s v \approx 0 ; b^{2} \approx 0$

$b \lambda e^{\lambda t}=\psi f\left(S^{(i)}\right) B^{(i)}+\psi f\left(S^{(i)}\right) b e^{\lambda t}$

$+\psi_{1} B^{(i)} \frac{s a e^{\lambda t}}{\left[a+S^{(i)}\right]^{2}}-\gamma\left[V^{(i)} B^{(i)}+V^{(i)} b e^{\lambda t}\right.$

$\left.+B^{(i)} v e^{\lambda t}\right]-B^{(i)}\left(B^{(i)} k_{1}+V^{(i)} k_{2}+S^{(i)} k_{3}\right)$

$-B^{(i)}\left(b k_{1}+v k_{2}+s k_{3}\right) e^{\lambda t}-\left(B^{(i)} k_{1}+V^{(i)} k_{2}\right.$

$\left.+S^{(i)} k_{3}\right) b e^{\lambda t}$

$b \lambda e^{\lambda t}=\psi f\left(S^{(i)}\right) B^{(i)}-\gamma V^{(i)} B^{(i)}-B^{(i)}\left(B^{(i)} k_{1}\right.$ $\left.+V^{(i)} k_{2}+S^{(i)} k_{3}\right)+\psi f\left(S^{(i)}\right) b e^{\lambda t}+\psi B^{(i)} \frac{s a e^{\lambda t}}{\left[a+S^{(i)}\right]^{2}}$ $-\gamma V^{(i)} b e^{\lambda t}-\gamma B^{(i)} v e^{\lambda t}-B^{(i)}\left(b k_{1}+v k_{2}+s k_{3}\right) e^{\lambda t}$ $-\left(B^{(i)} k_{1}+V^{(i)} k_{2}+S^{(i)} k_{3}\right) b e^{\lambda t}$

Any Chemostat fixed point: $\frac{d B}{d t}=0$ :

$$
\begin{aligned}
& \frac{d B}{d t}=0 \Rightarrow \psi f\left(S^{(i)}\right) B^{(i)}-\gamma V^{(i)} B^{(i)} \\
& -B^{(i)}\left[B^{(i)} k_{1}+V^{(i)} k_{2}+S^{(i)} k_{3}\right]=0
\end{aligned}
$$

$b \lambda e^{\lambda t}=\psi f\left(S^{(i)}\right) b e^{\lambda t}+\psi B^{(i)} \frac{s a e^{\lambda t}}{\left[a+S^{(i)}\right]^{2}}$

$-\gamma V^{(i)} b e^{\lambda t}-\gamma B^{(i)} v e^{\lambda t}-B^{(i)}\left(b k_{1}\right.$

$\left.+v k_{2}+s k_{3}\right) e^{\lambda t}-\left(B^{(i)} k_{1}+V^{(i)} k_{2}\right.$

$\left.+S^{(i)} k_{3}\right) b e^{\lambda t}$

$\psi f\left(S^{(i)}\right) b e^{\lambda t}+\psi B^{(i)} \frac{s a e^{\lambda t}}{\left[a+S^{(i)}\right]^{2}}-\gamma V^{(i)} b e^{\lambda t}$

$-\gamma B^{(i)} v e^{\lambda t}-B^{(i)}\left(b k_{1}+v k_{2}+s k_{3}\right) e^{\lambda t}$

$-\left(B^{(i)} k_{1}+V^{(i)} k_{2}+S^{(i)} k_{3}\right) b e^{\lambda t}-b \lambda e^{\lambda t}=0$

$s\left(\psi B^{(i)} \frac{a e^{\lambda t}}{\left[a+S^{(i)}\right]^{2}}-B^{(i)} k_{3} e^{\lambda t}\right)-v\left(B^{(i)} k_{2} e^{\lambda t}\right.$ $\left.+\gamma B^{(i)} e^{\lambda t}\right)+b\left\{\psi f\left(S^{(i)}\right) e^{\lambda t}-\gamma V^{(i)} e^{\lambda t}\right.$

$-B^{(i)} k_{1} e^{\lambda t}-\left(B^{(i)} k_{1}+V^{(i)} k_{2}+S^{(i)} k_{3}\right) e^{\lambda t}$

$\left.-\lambda e^{\lambda t}\right\}=0$

Divide above equation by $e^{\lambda t}$ and we get

$$
\begin{aligned}
& s\left(\psi B^{(i)} \frac{a}{\left[a+S^{(i)}\right]^{2}}-B^{(i)} k_{3}\right)-v\left(B^{(i)} k_{2}+\gamma B^{(i)}\right) \\
& +b\left\{\psi f\left(S^{(i)}\right)-\gamma V^{(i)}-B^{(i)} k_{1}-\left(B^{(i)} k_{1}\right.\right. \\
& \left.\left.+V^{(i)} k_{2}+S^{(i)} k_{3}\right)-\lambda\right\}=0
\end{aligned}
$$

We can summery our Chemostat three equations term of $s v$ and $b$ :

$$
\begin{aligned}
& s\left\{\left[1-S^{(i)}\right] k_{3}-\left[B^{(i)} k_{1}+V^{(i)} k_{2}+S^{(i)} k_{3}\right]\right. \\
& \left.-\psi \frac{a B^{(i)}}{\left[a+S^{(i)}\right]^{2}}-\lambda\right\}+v\left[1-S^{(i)}\right] k_{2}+b\left\{\left[1-S^{(i)}\right] k_{1}\right. \\
& \left.-\psi \frac{S^{(i)} a}{\left[a+S^{(i)}\right]^{2}}-\psi \frac{\left[S^{(i)}\right]^{2}}{\left[a+S^{(i)}\right]^{2}}\right\}=0
\end{aligned}
$$

$$
\begin{aligned}
& -s V^{(i)} k_{3}+v\left\{\gamma e^{-\lambda \tau} B^{(i)}-\varphi-\gamma B^{(i)} \frac{1}{\beta}\right. \\
& \left.-V^{(i)} k_{2}-\left[B^{(i)} k_{1}+V^{(i)} k_{2}+S^{(i)} k_{3}\right]-\lambda\right\} \\
& +b\left\{\gamma V^{(i)} e^{-\lambda \tau}-\gamma V^{(i)} \frac{1}{\beta}-V^{(i)} k_{1}\right\}=0
\end{aligned}
$$

$s\left(\psi B^{(i)} \frac{a}{\left[a+S^{(i)}\right]^{2}}-B^{(i)} k_{3}\right)-v\left(B^{(i)} k_{2}+\gamma B^{(i)}\right)$

$+b\left\{\psi f\left(S^{(i)}\right)-\gamma V^{(i)}-B^{(i)} k_{1}-\left(B^{(i)} k_{1}\right.\right.$

$$
\left.\left.+V^{(i)} k_{2}+S^{(i)} k_{3}\right)-\lambda\right\}=0
$$

246) The small increments Jacobian of our Chemostat system with control variables is as bellow:

$$
\left(\begin{array}{lll}
\Xi_{11} & \Xi_{12} & \Xi_{13} \\
\Xi_{21} & \Xi_{22} & \Xi_{23} \\
\Xi_{31} & \Xi_{32} & \Xi_{33}
\end{array}\right)\left(\begin{array}{l}
s \\
v \\
b
\end{array}\right)=0
$$

$\Xi_{11}=\left[1-S^{(i)}\right] k_{3}-\left[B^{(i)} k_{1}+V^{(i)} k_{2}+S^{(i)} k_{3}\right]$ $-\psi \frac{a B^{(i)}}{\left[a+S^{(i)}\right]^{2}}-\lambda$

$\Xi_{12}=\left[1-S^{(i)}\right] k_{2} ; \Xi_{13}=\left[1-S^{(i)}\right] k_{1}-\psi f\left(S^{(i)}\right)$

$\Xi_{21}=-V^{(i)} k_{3} ; \Xi_{22}=\gamma e^{-\lambda \tau} B^{(i)}-\varphi-\gamma B^{(i)} \frac{1}{\beta}$

$-V^{(i)} k_{2}-\left[B^{(i)} k_{1}+V^{(i)} k_{2}+S^{(i)} k_{3}\right]-\lambda$

$$
\begin{gathered}
\frac{S^{(i)} a}{\left[a+S^{(i)}\right]^{2}}+\frac{\left[S^{(i)}\right]^{2}}{\left[a+S^{(i)}\right]^{2}}=\frac{S^{(i)} a+\left[S^{(i)}\right]^{2}}{\left[a+S^{(i)}\right]^{2}} \\
\frac{S^{(i)} a}{\left[a+S^{(i)}\right]^{2}}+\frac{\left[S^{(i)}\right]^{2}}{\left[a+S^{(i)}\right]^{2}}=\frac{S^{(i)}}{\left[a+S^{(i)}\right]}=f\left(S^{(i)}\right) \\
\Xi_{23}=\gamma V^{(i)} e^{-\lambda \tau}-\gamma V^{(i)} \frac{1}{\beta}-V^{(i)} k_{1} \\
\Xi_{31}=\psi B^{(i)} \frac{a}{\left[a+S^{(i)}\right]^{2}}-B^{(i)} k_{3} \\
\Xi_{32}=-\left(B^{(i)} k_{2}+\gamma B^{(i)}\right) \\
\Xi_{33}=\psi f\left(S^{(i)}\right)-\gamma V^{(i)}-B^{(i)} k_{1} \\
-\left(B^{(i)} k_{1}+V^{(i)} k_{2}+S^{(i)} k_{3}\right)-\lambda
\end{gathered}
$$

$\left[1-S^{(i)}\right] k_{3}$

$-\left[B^{(i)} k_{1}\right.$

$\left.+V^{(i)} k_{2}+S^{(i)} k_{3}\right] \quad\left[1-S^{(i)}\right] k_{2}$

$\begin{array}{cll}-\psi \frac{a B^{(i)}}{\left[a+S^{(i)}\right]^{2}}-\lambda & & -\psi \frac{\left[S^{(i)}\right]^{2}}{\left[a+S^{(i)}\right]^{2}} \\ & \gamma e^{-\lambda \tau} B^{(i)} & \\ & -\varphi & \\ & -\gamma B^{(i)} \frac{1}{\beta} & \gamma V^{(i)} e^{-\lambda T} \\ -V^{(i)} k_{3} & -V^{(i)} k_{2} & -\gamma V^{(i)} \frac{1}{\beta} \\ & -\left[B^{(i)} k_{1}\right. & -V^{(i)} k_{1} \\ & +V^{(i)} k_{2} & \\ & \left.+S^{(i)} k_{3}\right] & \\ & -\lambda & \psi f\left(S^{(i)}\right) \\ & & -\gamma V^{(i)} \\ & & -B^{(i)} k_{1} \\ \left(\psi B^{(i)} \frac{a}{\left[a+S^{(i)}\right]^{2}}\right. & -B^{(i)}\left(k_{2}+\gamma\right) & -\left(B^{(i)} k_{1}\right. \\ \left.-B^{(i)} k_{3}\right) & & +V^{(i)} k_{2} \\ & & \left.+S^{(i)} k_{3}\right) \\ & & -\lambda\end{array}$

$\left[1-S^{(i)}\right] k_{1}$

$$
\begin{aligned}
& A-\lambda I=\left(\begin{array}{ccc}
\Xi_{11} & \Xi_{12} & \Xi_{13} \\
\Xi_{21} & \Xi_{22} & \Xi_{23} \\
\Xi_{31} & \Xi_{32} & \Xi_{33}
\end{array}\right) ; \operatorname{det}|A-\lambda I|=0 \\
& A=\left(\begin{array}{lll}
\Upsilon_{11} & \Upsilon_{12} & \Upsilon_{13} \\
\Upsilon_{21} & \Upsilon_{22} & \Upsilon_{23} \\
\Upsilon_{31} & \Upsilon_{32} & \Upsilon_{33}
\end{array}\right)
\end{aligned}
$$


Ofer Aluf; Dynamic of Bacterial and Phage Populations Maintained in Chemostat System with Dilution Control Parameter, Transactions on Networks and Communications, Volume 4 No. 3, June (2016); pp: 1-21

$$
\begin{aligned}
& \Upsilon_{11}=\left[1-S^{(i)}\right] k_{3}-\left[B^{(i)} k_{1}+V^{(i)} k_{2}+S^{(i)} k_{3}\right] \\
& -\psi \frac{a B^{(i)}}{\left[a+S^{(i)}\right]^{2}} ; \Upsilon_{12}=\left[1-S^{(i)}\right] k_{2} \\
& \Upsilon_{13}=\left[1-S^{(i)}\right] k_{1}-\psi f\left(S^{(i)}\right) ; \Upsilon_{21}=-V^{(i)} k_{3} \\
& \Upsilon_{22}=\gamma e^{-\lambda \tau} B^{(i)}-\varphi-\gamma B^{(i)} \frac{1}{\beta}-V^{(i)} k_{2} \\
& -\left[B^{(i)} k_{1}+V^{(i)} k_{2}+S^{(i)} k_{3}\right] \\
& \Upsilon_{23}=\gamma V^{(i)} e^{-\lambda \tau}-\gamma V^{(i)} \frac{1}{\beta}-V^{(i)} k_{1} \\
& \Upsilon_{31}=\left(\psi B^{(i)} \frac{a}{\left[a+S^{(i)}\right]^{2}}-B^{(i)} k_{3}\right) \\
& \Upsilon_{32}=B^{(i)}\left(k_{2} \mid \gamma\right) \\
& \Upsilon_{33}=\psi f\left(S^{(i)}\right)-\gamma V^{(i)}-B^{(i)} k_{1} \\
& -\left(B^{(i)} k_{1}+V^{(i)} k_{2}+S^{(i)} k_{3}\right) \\
& \operatorname{det}|A-\lambda I|=0 \\
& \left(\Upsilon_{11}-\lambda\right) \operatorname{det}\left(\begin{array}{cc}
\left(\Upsilon_{22}-\lambda\right) & \Upsilon_{23} \\
\Upsilon_{32} & \left(\Upsilon_{33}-\lambda\right)
\end{array}\right) \\
& -\Upsilon_{12} \operatorname{det}\left(\begin{array}{cc}
\Upsilon_{21} & \Upsilon_{23} \\
\Upsilon_{31} & \left(\Upsilon_{33}-\lambda\right)
\end{array}\right) \\
& +\Upsilon_{13} \operatorname{det}\left(\begin{array}{cc}
\Upsilon_{21} & \left(\Upsilon_{22}-\lambda\right) \\
\Upsilon_{31} & \Upsilon_{32}
\end{array}\right)=0 \\
& \left(\Upsilon_{11}-\lambda\right)\left\{\left(\Upsilon_{22}-\lambda\right)\left(\Upsilon_{33}-\lambda\right)-\Upsilon_{32} \Upsilon_{23}\right\} \\
& -\Upsilon_{12}\left\{\Upsilon_{21}\left(\Upsilon_{33}-\lambda\right)-\Upsilon_{31} \Upsilon_{23}\right\} \\
& +\Upsilon_{13}\left\{\Upsilon_{21} \Upsilon_{32}-\Upsilon_{31}\left(\Upsilon_{22}-\lambda\right)\right\}=0 \\
& \left(\Upsilon_{11}-\lambda\right)\left\{\Upsilon_{22} \Upsilon_{33}-\Upsilon_{32} \Upsilon_{23}\right. \\
& \left.-\lambda\left(\Upsilon_{22}+\Upsilon_{33}\right)+\lambda^{2}\right\} \\
& -\Upsilon_{12}\left\{\Upsilon_{21} \Upsilon_{33}-\Upsilon_{31} \Upsilon_{23}-\Upsilon_{21} \lambda\right\} \\
& +\Upsilon_{13}\left\{\Upsilon_{21} \Upsilon_{32}-\Upsilon_{31} \Upsilon_{22}+\Upsilon_{31} \lambda\right\}=0 \\
& \Upsilon_{11} \Upsilon_{22} \Upsilon_{33}-\Upsilon_{11} \Upsilon_{32} \Upsilon_{23} \\
& -\lambda \Upsilon_{11}\left(\Upsilon_{22}+\Upsilon_{33}\right)+\Upsilon_{11} \lambda^{2} \\
& -\lambda \Upsilon_{22} \Upsilon_{33}+\lambda \Upsilon_{32} \Upsilon_{23}+\lambda^{2}\left(\Upsilon_{22}+\Upsilon_{33}\right) \\
& -\lambda^{3}-\Upsilon_{12} \Upsilon_{21} \Upsilon_{33}+\Upsilon_{12} \Upsilon_{31} \Upsilon_{23}+\Upsilon_{12} \Upsilon_{21} \lambda \\
& +\Upsilon_{13} \Upsilon_{21} \Upsilon_{32}-\Upsilon_{13} \Upsilon_{31} \Upsilon_{22}+\Upsilon_{13} \Upsilon_{31} \lambda=0 \\
& -\lambda^{3}+\lambda^{2}\left[\Upsilon_{11}+\Upsilon_{22}+\Upsilon_{33}\right]+\lambda\left[\Upsilon_{32} \Upsilon_{23}\right. \\
& -\Upsilon_{22} \Upsilon_{33}+\Upsilon_{12} \Upsilon_{21}-\Upsilon_{11}\left(\Upsilon_{22}+\Upsilon_{33}\right) \\
& \left.+\Upsilon_{13} \Upsilon_{31}\right]+\Upsilon_{11} \Upsilon_{22} \Upsilon_{33}-\Upsilon_{11} \Upsilon_{32} \Upsilon_{23} \\
& -\Upsilon_{12} \Upsilon_{21} \Upsilon_{33}+\Upsilon_{12} \Upsilon_{31} \Upsilon_{23}+\Upsilon_{13} \Upsilon_{21} \Upsilon_{32} \\
& -\Upsilon_{13} \Upsilon_{31} \Upsilon_{22}=0
\end{aligned}
$$

We define the following parameters:

$$
\begin{aligned}
& \Xi_{3}=-1 ; \Xi_{2}\left(\tau, k_{1}, k_{2}, k_{3}\right)=\Upsilon_{11}+\Upsilon_{22}+\Upsilon_{33} \\
& \Xi_{1}\left(\tau, k_{1}, k_{2}, k_{3}\right)=\Upsilon_{32} \Upsilon_{23}-\Upsilon_{22} \Upsilon_{33}+\Upsilon_{12} \Upsilon_{21} \\
& -\Upsilon_{11}\left(\Upsilon_{22}+\Upsilon_{33}\right)+\Upsilon_{13} \Upsilon_{31} \\
& \\
& \Xi_{0}=\Upsilon_{11} \Upsilon_{22} \Upsilon_{33}-\Upsilon_{11} \Upsilon_{32} \Upsilon_{23}-\Upsilon_{12} \Upsilon_{21} \Upsilon_{33} \\
& +\Upsilon_{12} \Upsilon_{31} \Upsilon_{23}+\Upsilon_{13} \Upsilon_{21} \Upsilon_{32}-\Upsilon_{13} \Upsilon_{31} \Upsilon_{22}
\end{aligned}
$$

We get our Chemostat system with control variables characteristic equation:

$$
\begin{aligned}
& D\left(\lambda, \tau, k_{1}, k_{2}, k_{3}\right)=\sum_{j=0}^{3} \lambda^{j} \Xi_{j}\left(\tau, k_{1}, k_{2}, k_{3}\right) \\
& \tau>0 ; k_{1}>0, k_{2}>0, k_{3}>0
\end{aligned}
$$

We have three Chemostat stability cases:

(265) (1) $\tau>0 ; k_{1}=k ; k_{2}=k_{3}=0$

(2) $\tau>0 ; k_{1}=k_{2}=k ; k_{3}=0$

(3) $\tau>0 ; k_{1}=k_{2}=k_{3}=k$

We need to get characteristics equations for all above stability analysis cases. We study the occurrence of any possible stability switching resulting from the increase of value of the time delay $\tau$ and $k_{1}, k_{2}, k_{3}$ parameters for the general characteristic equation: $D\left(\lambda, \tau, k_{1}, k_{2}, k_{3}\right)$ or $D(\lambda, \tau, k)$. If we choose $\tau$ and $k$ parameters then the (267) following:

$$
D(\lambda, \tau, k)=P_{n}(\lambda, \tau, k)+Q_{m}(\lambda, \tau, k) e^{-\lambda \tau}
$$

IV. CHEMOSTAT SYSTEM WITH CONTROL PARAMETER AND DELAYED VARIABLES IN TIME CHARACTERISTIC EQUATION We choose to analyze for first case: $\tau>0 ; k_{1}=k$ and $k_{2}=k_{3}=0$. We need to get characteristics equation:

$$
\begin{aligned}
& D(\lambda, \tau, k)=P_{n}(\lambda, \tau, k)+Q_{m}(\lambda, \tau, k) e^{-\lambda \tau} \\
& \Upsilon_{11}=-B^{(i)}\left\{k+\psi \frac{a}{\left[a+S^{(i)}\right]^{2}}\right\} ; \Upsilon_{12}=0 \\
& \Upsilon_{13}=\left[1-S^{(i)}\right] k-\psi f\left(S^{(i)}\right) ; \Upsilon_{21}=0 \\
& \Upsilon_{22}=B^{(i)}\left[\gamma e^{-\lambda \tau}-\varphi-\gamma \frac{1}{\beta}-k\right] \\
& \Upsilon_{23}=\gamma V^{(i)} e^{-\lambda \tau}-\gamma V^{(i)} \frac{1}{\beta}-V^{(i)} k \\
& \Upsilon_{23}=V^{(i)}\left[\gamma e^{-\lambda \tau}-\gamma \frac{1}{\beta}-k\right] \\
& \Upsilon_{31}=\psi B^{(i)} \frac{a}{\left[a+S^{(i)}\right]^{2}} ; \Upsilon_{32}=-B^{(i)} \gamma \\
& \Upsilon_{33}=\psi f\left(S^{(i)}\right)-\gamma V^{(i)}-2 B^{(i)} k \\
& \Xi_{3}=-1 ; \Xi_{2}=\Upsilon_{11}+\Upsilon_{22}+\Upsilon_{33} \\
& \Xi_{2}=-B^{(i)} \psi \frac{a}{\left[a+S^{(i)}\right]^{2}}-B^{(i)} \varphi-\gamma B^{(i)} \frac{1}{\beta} \\
& +\psi f\left(S^{(i)}\right)-\gamma V^{(i)}-4 B^{(i)} k+B^{(i)} \gamma e^{-\lambda \tau} \\
& \Xi_{21}=-B^{(i)} \psi \frac{a}{\left[a+S^{(i)}\right]^{2}}-B^{(i)} \varphi-\gamma B^{(i)} \frac{1}{\beta} \\
& +\psi f\left(S^{(i)}\right)-\gamma V^{(i)}-4 B^{(i)} k \\
& \Xi_{22}=\gamma B^{(i)} ; \Xi_{2}=\Xi_{21}+\Xi_{22} e^{-\lambda \tau} \\
& \begin{array}{l}
\Xi_{1} \mid \Upsilon_{12}=0, \Upsilon_{21}=0=\Upsilon_{32} \Upsilon_{23}-\Upsilon_{22} \Upsilon_{33} \\
-\Upsilon_{11}\left(\Upsilon_{22}+\Upsilon_{33}\right)+\Upsilon_{13} \Upsilon_{31}
\end{array}
\end{aligned}
$$

$\Xi_{1}=-B^{(i)} \gamma V^{(i)}\left[\gamma e^{-\lambda \tau}-\gamma \frac{1}{\beta}-k\right]$ $-B^{(i)}\left[\gamma e^{-\lambda \tau}-\varphi-\gamma \frac{1}{\beta}-k\right]\left[\psi f\left(S^{(i)}\right)-\gamma V^{(i)}\right.$

$\left.-2 B^{(i)} k\right]+B^{(i)}\left\{k+\psi \frac{a}{\left[a+S^{(i)}\right]^{2}}\right\}\left\{B^{(i)} \gamma e^{-\lambda \tau}\right.$

$-B^{(i)}\left(\varphi+\gamma \frac{1}{B}+k\right)+\psi f\left(S^{(i)}\right)-\gamma V^{(i)}$

$\left.-2 B^{(i)} k\right\}+\left\{\left[1-S^{(i)}\right] k\right.$

$\left.-\psi f\left(S^{(i)}\right)\right\}\left\{\psi B^{(i)} \frac{a}{\left[a+S^{(i)}\right]^{2}}\right\}$

$\Xi_{1}=-B^{(i)} \gamma V^{(i)} \gamma e^{-\lambda \tau}+B^{(i)} \gamma V^{(i)}\left(\gamma \frac{1}{\beta}+k\right)$ $-B^{(i)}\left[\psi f\left(S^{(i)}\right)-\gamma V^{(i)}-2 B^{(i)} k\right] \gamma e^{-\lambda \tau}$

$+B^{(i)}\left(\varphi+\gamma \frac{1}{\beta}+k\right)\left[\psi f\left(S^{(i)}\right)-\gamma V^{(i)}-2 B^{(i)} k\right]$

$+B^{(i)}\left\{k+\psi \frac{a}{\left[a+S^{(i)}\right]^{2}}\right\}\left(B^{(i)}\left[\gamma e^{-\lambda \tau}\right.\right.$

$\left.\left.-\left(\varphi+\gamma \frac{1}{\beta}+k\right)\right]+\psi f\left(S^{(i)}\right)-\gamma V^{(i)}-2 B^{(i)} k\right)$

$+\left\{\left[1-S^{(i)}\right] k-\psi f\left(S^{(i)}\right)\right\}\left\{\psi B^{(i)} \frac{a}{\left[a+S^{(i)}\right]^{2}}\right\}$ 


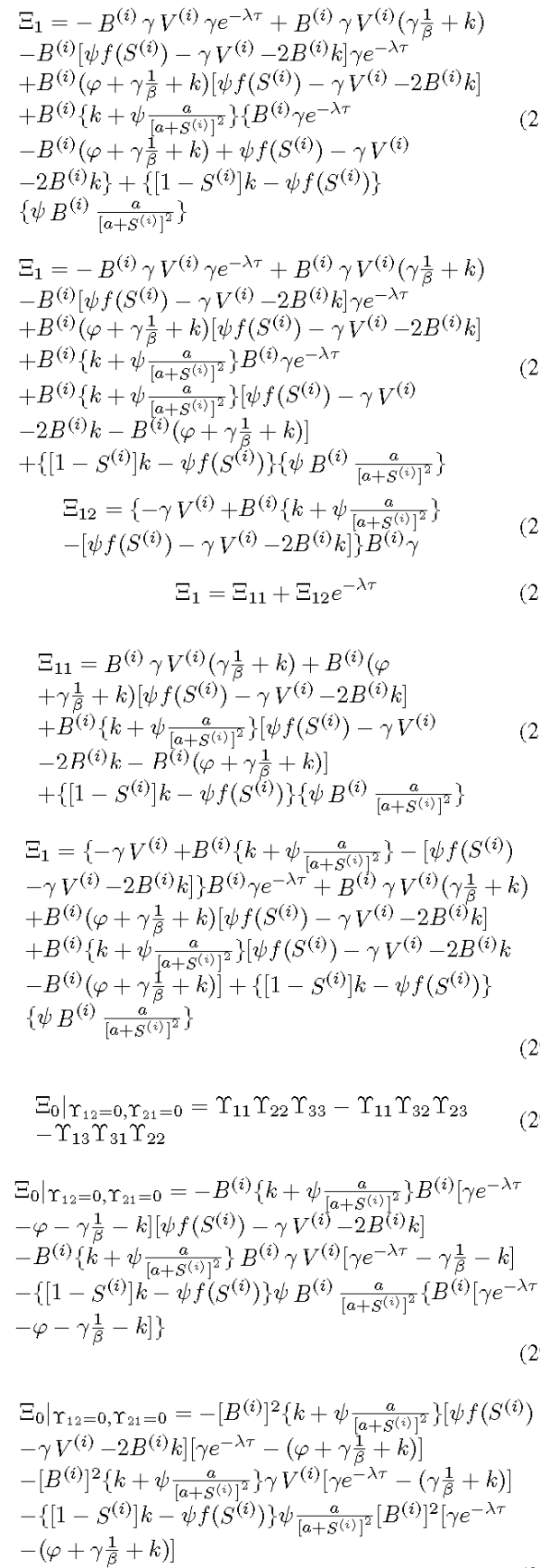

$\Xi_{0} \mid \Upsilon_{12}=0, \Upsilon_{21}=0=-\left[B^{(i)}\right]^{2}\left\{k+\psi \frac{a}{\left[a+S^{(i)}\right]^{2}}\right\}\left[\psi f\left(S^{(i)}\right)\right.$ $\left.-\gamma V^{(i)}-2 B^{(i)} k\right]\left[\gamma e^{-\lambda \tau}-\left(\varphi+\gamma \frac{1}{\beta}+k\right)\right]$ $-\left[B^{(i)}\right]^{2}\left\{k+\psi \frac{a}{\left[a+S^{(i)}\right]^{2}}\right\} \gamma V^{(i)}\left[\gamma e^{-\lambda \tau}-\left(\gamma \frac{1}{\beta}+k\right)\right]$

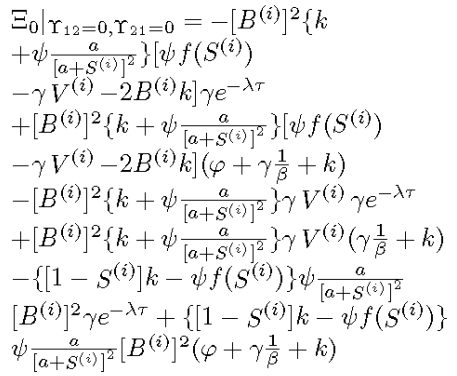

$\Xi_{02} \mid \Upsilon_{12}=0, \Upsilon_{21}=0=-\left(\left\{\left[1-S^{(2)}\right] k-\psi f\left(S^{(i)}\right)\right\}\right.$ $\psi \frac{a}{\left[a+S^{(i)}\right]^{2}}+\left\{k+\psi \frac{a}{\left[a+S^{(i)}\right]^{2}}\right\}\left[\psi f\left(S^{(i)}\right)-\gamma V^{(i)}\right.$ $\left.\left.-2 B^{(i)} k\right]+\left\{k+\psi \frac{a}{\left[a+S^{(i)}\right]^{2}}\right\} \gamma V^{(i)}\right)\left[B^{(i)}\right]^{2} \gamma$

$\Xi_{01} \mid \Upsilon_{12=0, \Upsilon_{21}=0}=\left[B^{(i)}\right]^{2}\left\{k+\psi \frac{a}{\left[a+S^{(i)}\right]^{2}}\right.$ \}$\left[\psi f\left(S^{(i)}\right)-\gamma V^{(i)}-2 B^{(i)} k\right]\left(\varphi+\gamma \frac{1}{\beta}+k\right)$ $+\left[B^{(i)}\right]^{2}\left\{h+\psi \frac{a}{\left[a+S^{(i)}\right]^{2}}\right\} \gamma V^{(i)}\left(\gamma \frac{1}{\beta}+k\right)$ $+\left\{\left[1-S^{(i)}\right] k-\psi f\left(S^{(i)}\right)\right\} \psi \frac{a}{\left[a+S^{(i)}\right]^{2}}\left[B^{(i)}\right]^{2}$ $\left(\varphi+\gamma \frac{1}{\beta}+h\right)$

$$
\begin{aligned}
& \Xi_{0}\left|\Upsilon_{12}=0, \Upsilon_{21}=0=\Xi_{01}\right| \Upsilon_{12}=0, \Upsilon_{21}=0 \\
& +\Xi_{02} \mid \Upsilon_{12}=0, \Upsilon_{21}=0
\end{aligned}
$$

$$
\begin{aligned}
& D(\lambda, \tau, k)=\sum_{j=0}^{3} \lambda^{j} \Xi_{j}(\tau, k) \\
& \tau>0 ; k_{1}=k>0, k_{2}=0, k_{3}=0 \\
& D(\lambda, \tau, k)=\sum_{j=0}^{3} \lambda^{j} \Xi_{j}(\tau, k)=\lambda^{3} \Xi_{3}(\tau, k) \\
& +\lambda^{2} \Xi_{2}(\tau, k)+\lambda \Xi_{1}(\tau, k)+\Xi_{0}(\tau, k) \\
& \left.D(\lambda, \tau, k)\right|_{\Upsilon_{12}=0, \Upsilon_{21}=0}=-\lambda^{3}+\lambda^{2}\left(\Xi_{21}\right. \\
& \left.+\Xi_{22} e^{-\lambda \tau}\right)+\lambda\left(\Xi_{11}+\Xi_{12} e^{-\lambda \tau}\right)+\Xi_{01} \\
& +\Xi_{02} e^{-\lambda \tau} \\
& \left.D(\lambda, \tau, k)\right|_{\Upsilon_{12}=0, \Upsilon_{21}=0}=-\lambda^{3}+\lambda^{2} \Xi_{21} \\
& +\lambda \Xi_{11}+\Xi_{01}+\left[\lambda \Xi_{12}+\Xi_{02}+\Xi_{22}\right] e^{-\lambda \tau}
\end{aligned}
$$

$\Xi_{21}=\Xi_{21}(k) ; \Xi_{22} ; \Xi_{11}=\Xi_{11}(k) ; \Xi_{12}=\Xi_{12}(k)$ $\Xi_{01}=\Xi_{01}(k) ; \Xi_{02}=\Xi_{02}(k)$ 


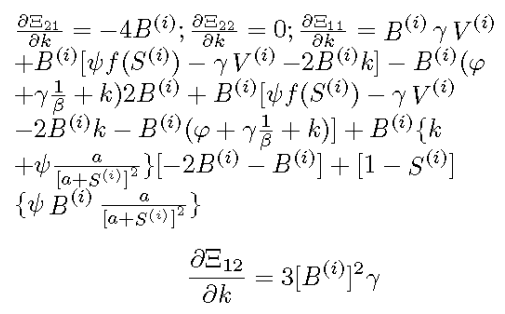

$\frac{\partial \Xi_{01}}{\partial k}=\left[B^{(i)}\right]^{2}\left[\psi f\left(S^{(i)}\right)-\gamma V^{(i)}-2 B^{(i)} k\right]\left(\varphi+\gamma \frac{1}{\beta}+k\right)$ $+\left[B^{(i)}\right]^{2}\left\{k+\psi \frac{a}{\left[a+S^{(i)}\right]^{2}}\right\}\left(-2 B^{(i)}\right)\left(\varphi+\gamma \frac{1}{\beta}+k\right)$ $+\left[B^{(i)}\right]^{2}\left\{k+\psi \frac{a}{\left[a+S^{(i)}\right]^{2}}\right\}\left[\psi f\left(S^{(i)}\right)-\gamma V^{(i)}-2 B^{(i)} k\right]$ $+\left[B^{(i)}\right]^{2} \gamma V^{(i)}\left(\gamma \frac{1}{\beta}+k\right)+\left[B^{(i)}\right]^{2}\left\{k+\psi \frac{a}{\left[a+S^{(i)}\right]^{2}}\right\} \gamma V^{(i)}$ $+\left[1-S^{(i)}\right] \psi \frac{a}{\left[a+S^{(i)}\right]^{2}}\left[B^{(i)}\right]^{2}\left(\varphi+\gamma \frac{1}{\beta}+k\right)$ $+\left\{\left[1-S^{(i)}\right] k-\psi f\left(S^{(i)}\right)\right\} \psi \frac{a}{\left[a+\left.S^{(i)}\right|^{2}\right.}\left[B^{(i)}\right]^{2}$

$\frac{\partial \Xi_{02}}{\partial \grave{k}}=-\left(\left[1-S^{(i)}\right] \psi \frac{a}{\left[a+S^{(i)}\right]^{2}}+\left[\psi f\left(S^{(i)}\right)\right.\right.$ $\left.-\gamma V^{(i)}-2 B^{(i)} k\right]-\left\{k+\psi \frac{a}{\left[a+S^{(i)}\right]^{2}}\right\} 2 B^{(i)}$ $\left.+\gamma V^{(i)}\right)\left[B^{(i)}\right]^{2} \gamma$

$\left.D(\lambda, \tau, k)\right|_{\Upsilon_{12}=0, \Upsilon_{21}=0}=-\lambda^{3}+\lambda^{2} \Xi_{21}+\lambda \Xi_{11}$ $+\Xi_{01}+\left[\lambda \Xi_{12}+\Xi_{02}+\Xi_{22}\right] e^{-\lambda \tau}$

$P_{n}(\lambda, k)=-\lambda^{3}+\lambda^{2} \Xi_{21}+\lambda \Xi_{11}+\Xi_{01}$ $Q_{m}(\lambda, k)=\lambda \Xi_{12}+\left(\Xi_{02}+\Xi_{22}\right)$$$
D(\lambda, \tau, k)=P_{n}(\lambda, k)+Q_{m}(\lambda, k) e^{-\lambda \tau}
$$$$
n=3 ; m=1 ; n>m
$$

The expression for $P_{n}(\lambda, k)$ is

$$
\begin{aligned}
& P_{n}(\lambda, k)=\sum_{l=0}^{n=3} p_{l}(k) \lambda^{l}=p_{0}(k)+p_{1}(k) \lambda \\
& +p_{2}(k) \lambda^{2}+p_{3}(k) \lambda^{3}
\end{aligned}
$$

$p_{0}(k)=\Xi_{01} ; p_{1}(k)=\Xi_{11} ; p_{2}(k)=\Xi_{21} ; p_{3}(k)=-1$

The expression for $Q_{m}(\lambda, k)$ is

$$
\begin{gathered}
Q_{m}(\lambda, k)=\sum_{l=0}^{m=1} q_{l}(k) \lambda^{l}=q_{0}(k)+q_{1}(k) \lambda \\
q_{0}(k)=\Xi_{02}+\Xi_{22} ; q_{1}(k)=\Xi_{12}
\end{gathered}
$$

The homogeneous system for $S, V, B$ leads to a characteristic equation for the eigenvalue $\lambda$ having the form

$$
\begin{gathered}
P(\lambda, k)+Q(\lambda, k) e^{-\lambda \tau}=0 \\
P(\lambda, k)+Q(\lambda, k) e^{-\lambda \tau}=0 \\
P(\lambda, k)=\sum_{l=0}^{3} a_{l} \lambda^{l} ; Q(\lambda, k)=\sum_{l=0}^{1} c_{l} \lambda^{l}
\end{gathered}
$$

The coefficients $\left\{a_{l}(k), c_{l}(k)\right\} \in R$ depend on $k$ (active washout rate balance parameter), other parameters kept as a constant.

$$
\begin{aligned}
& a_{0}(k)=\Xi_{01} ; a_{1}(k)=\Xi_{11} ; a_{2}(k)=\Xi_{21} \\
& a_{3}(k)=-1 ; c_{0}(k)=\Xi_{02}+\Xi_{22} ; c_{1}(k)=\Xi_{12}
\end{aligned}
$$

The designation of the variation argument $(k)$ will subsequently be omitted from $P, Q, a_{l}$, and $c_{l}$. The coefficients $a_{l}, c_{l}$ are continuous, and differential functions of their arguments, and direct substitution shows that $a_{0}+c_{0} \neq 0$ for $k \in R_{+}$; that is, $\lambda=0$ is not of the following:

$$
\begin{aligned}
& P(\lambda, k)=P(\lambda) ; Q(\lambda, k)=Q(\lambda) \\
& P(\lambda, k)+Q(\lambda, k) e^{-\lambda \tau}=0
\end{aligned}
$$

Furthermore, $P(\lambda), Q(\lambda)$ are analytic functions of $\lambda$, for which the following requirements of the analysis $[\mathrm{BK}]$ can also be verified in the present case:

(1) If $\lambda=i \omega, \omega \in$, then $P(i \omega)+Q(i \omega) \neq 0$.

(2) $|Q(\lambda) / P(\lambda)|$ is bounded for $|\lambda| \rightarrow \infty, \operatorname{Re} \lambda \geq 0$. No roots bifurcation from $\infty$.

(306) (3) $F(\omega)=|P(i \omega)|^{2}-|Q(i \omega)|^{2}$ has a finite number of zeros. Indeed, this is a polynomial in $\omega$.

(4) Each positive root $\omega(k)$ of $F(\omega)=0$ is continuous and differentiable with respect to $\mathrm{k}$ parameter.

We assume that $P_{n}(\lambda, k)$ and $Q_{m}(\lambda, k)$ cannot have common imaginary roots. That is for any real number $\omega$ :

$$
P_{n}(\lambda=i \omega, k)+Q_{m}(\lambda=i \omega, k) \neq 0
$$

$$
\begin{gathered}
P_{n}(\lambda=i \omega, k)=-\omega^{2} \Xi_{21}+\Xi_{01}+i \omega\left[\Xi_{11}+\omega^{2}\right] \\
Q_{m}(\lambda=i \omega, k)=i \omega \Xi_{12}+\left(\Xi_{02}+\Xi_{22}\right) \\
P_{n}(\lambda=i \omega, k)=-\omega^{2} \Xi_{21}+\Xi_{01}+i \omega\left[\Xi_{11}+\omega^{2}\right] \\
Q_{m}(\lambda=i \omega, k)=i \omega \Xi_{12}+\left(\Xi_{02}+\Xi_{22}\right) \\
|Q(i \omega, k)|^{2}=\omega^{2} \Xi_{12}^{2}+\left(\Xi_{02}+\Xi_{22}\right)^{2}
\end{gathered}
$$

$$
\begin{aligned}
& F(\omega, k)=|P(i \omega, k)|^{2}-|Q(i \omega, k)|^{2}=\omega^{6} \\
& +\omega^{4}\left[\Xi_{21}^{2}+2 \Xi_{11}\right]+\omega^{2}\left[\Xi_{11}^{2}-2 \Xi_{21} \Xi_{01}\right]+\Xi_{01}^{2}
\end{aligned}
$$

$$
F(\omega, k)=|P(i \omega, k)|^{2}-|Q(i \omega, k)|^{2}=\omega^{6}
$$$$
+\omega^{4}\left[\Xi_{21}^{2}+2 \Xi_{11}\right]+\omega^{2}\left(\Xi_{11}^{2}-2 \Xi_{21} \Xi_{01}-\Xi_{12}^{2}\right)
$$$$
+\Xi_{01}^{2}-\left(\Xi_{02}+\Xi_{22}\right)^{2}
$$

We define the following parameters for simplicity:

$$
\begin{aligned}
& \Phi_{0}=\Xi_{01}^{2}-\left(\Xi_{02}+\Xi_{22}\right)^{2} \\
& \Phi_{2}=\Xi_{11}^{2}-2 \Xi_{21} \Xi_{01}-\Xi_{12}^{2} \\
& \Phi_{4}=\Xi_{21}^{2}+2 \Xi_{11} ; \Phi_{6}=1
\end{aligned}
$$

Hence $F(\omega, k)=0$ implies $\sum_{l=0}^{3} \Phi_{2 l}(k) \omega^{2 l}=0$ and its roots are given by solving the polynomial. 


$$
\begin{aligned}
& P_{R}(i \omega, k)=-\omega^{2} \Xi_{21}+\Xi_{01} \\
& P_{I}(i \omega, k)=\omega\left[\Xi_{11}+\omega^{2}\right] \\
& \mathrm{Q}_{R}(i \omega, k)=\Xi_{02}+\Xi_{22} \\
& \mathrm{Q}_{I}(i \omega, k)=\omega \Xi_{12}
\end{aligned}
$$

Hence

$$
\begin{aligned}
& \sin \theta(\tau) \\
& =\frac{-P_{R}(i \omega, k) Q_{I}(i \omega, k)+P_{I}(i \omega, k) Q_{R}(i \omega, k)}{|Q(i \omega)|^{2}} \\
& \cos \theta(\tau) \\
& =-\frac{P_{R}(i \omega, k) Q_{R}(i \omega, k)+P_{I}(i \omega, k) Q_{I}(i \omega, k)}{|Q(i \omega)|^{2}}
\end{aligned}
$$

Which jointly with $F(\omega, k)=0 \Rightarrow \sum_{l=0}^{3} \Phi_{2 l}(k) \omega^{2 l}=0$

That is continuous and differentiable in $\mathrm{k}$ parameter. We get the following general characteristic equation.

$$
\begin{aligned}
& D(\lambda, \tau, k)=P_{n}(\lambda, k)+Q_{m}(\lambda, k) e^{-\lambda \tau} \\
& n=3 ; m=1 ; n>m
\end{aligned}
$$

We use different parameters terminology from our last characteristics parameters definition:

$$
p_{l}(k) \rightarrow a_{l} ; q_{l}(k) \rightarrow c_{l} ; n=3 ; m=1 ; n>m
$$

Additionally $P_{n}(\lambda, k) \rightarrow P(\lambda) ; Q_{m}(\lambda, k) \rightarrow Q(\lambda)$

$$
\begin{aligned}
P(\lambda) & =\sum_{l=0}^{3} a_{l} \lambda^{l} ; Q(\lambda)=\sum_{l=0}^{1} c_{l} \lambda^{l} \\
P(\lambda) & =-\lambda^{3}+\lambda^{2} \Xi_{21}+\lambda \Xi_{11}+\Xi_{01} \\
Q(\lambda) & =\lambda \Xi_{12}+\left(\Xi_{02}+\Xi_{22}\right)
\end{aligned}
$$

$n, m \in N_{0} ; n>m$ and $a_{l}, c_{l}: R_{+0} \rightarrow R$ are continuous and differentiable function of $k$ such that $a_{0}+c_{0} \neq 0$. In the following "- " denotes complex and conjugate. $P(\lambda), Q(\lambda)$ are analytic functions in $\lambda$ and differentiable in $k$ parameter. The coefficients $\left\{a_{l}(k, \ldots)\right.$ and $\left.c_{l}(k, \ldots)\right\} \in R$ depend on Chemostat with control variables system's $k \ldots$ values. The designation of the variation arguments $(k, \ldots)$ will subsequently be omitted from $P, Q, a_{l}, c_{l}$. The coefficients $a_{l}, c_{l}$ are continues and differentiable function of their arguments, and direct substitution shows that $a_{0}+c_{0} \neq 0 ; \Xi_{01}+\Xi_{02}+\Xi_{22} \neq 0$. In addition, since the coefficients in $P$ and $Q$ are real, we have $\overline{P(-i \omega)}=P(i \omega)$ and $\overline{Q(-i \omega)}=Q(i \omega)$ thus $\lambda=i \omega, \omega>0$ may be on eigenvalue of characteristic equation. The analysis consists in identifying the roots of characteristics equation situated on the imaginary axis of the complex $\lambda$ - plane, whereby increasing the parameters $k, \ldots \operatorname{Re} \lambda$ may, at the crossing, change its sign from (-) to $(+)$, that is, from a stable focus $E^{*}$ to an unstable one, or vice versa. This feature may be further assessed by examining the sign of the partial derivatives with respect to $k, \ldots$, Chemostat system parameters [2] [3].

$$
\begin{aligned}
& \Lambda^{-1}(k)=\left(\frac{\partial \mathrm{Re} \lambda}{\partial k}\right)_{\lambda=i \omega} \\
& \varphi, \gamma, \beta, \psi, \ldots=\text { const } ; \omega \in R_{+}
\end{aligned}
$$

If $\Lambda(\mathrm{k})>0$ (or $<0$ ), then the crossing proceeds from $(-)$ to $(+)$ (or from $(+)$ to $(-)$ ), respectively.

$$
\begin{aligned}
& F(\omega, k)=|P(i \omega, k)|^{2}-|Q(i \omega, k)|^{2} \\
& F(\omega, k)=\sum_{l=0}^{3} \Phi_{2 l}(k) \omega^{2 l} \\
& F(\omega, k)=\Phi_{0}(k)+\Phi_{2}(k) \omega^{2} \\
& +\Phi_{4}(k) \omega^{4}+\Phi_{6}(k) \omega^{6}
\end{aligned}
$$

Hence $F(\omega, \tau)=0$ implies $\sum_{k=0}^{4} \Phi_{2 k} \omega^{2 k}=0$ When writing $P(\lambda)=P_{R}(\lambda)+i P_{I}(\lambda)$ and $Q(\lambda)=Q_{R}(\lambda)+i Q_{I}(\lambda)$, and inserting $\lambda=i \omega$ into our system's characteristic equation, $\omega$ must satisfy the following:

$$
\begin{aligned}
& \sin \omega \tau=g(\omega)=\frac{-P_{R}(i \omega) Q_{I}(i \omega)+P_{I}(i \omega) Q_{R}(i \omega)}{|Q(i \omega)|^{2}} \\
& \cos \omega \tau=h(\omega)=-\frac{P_{R}(i \omega) Q_{R}(i \omega)+P_{I}(i \omega) Q_{I}(i \omega)}{|Q(i \omega)|^{2}}
\end{aligned}
$$

Where $|Q(i \omega)|^{2} \neq 0$ in view of requirement (a) above, and $(g, h) \in R$. Furthermore, it follows above $\sin \omega \tau$ and $\cos \omega \tau$ equations that, by squaring and adding the sides, $\omega$ must be a positive root of $F(\omega)=|P(i \omega)|^{2}-|Q(i \omega)|^{2}=0$.

$$
\begin{aligned}
& \sin \theta(\tau)=\frac{-P_{R}(i \omega) Q_{I}(i \omega)+P_{I}(i \omega) Q_{R}(i \omega)}{|Q(i \omega)|^{2}} \\
& \cos \theta(\tau)=-\frac{P_{R}(i \omega) Q_{R}(i \omega)+P_{I}(i \omega) Q_{I}(i \omega)}{|Q(i \omega)|^{2}}
\end{aligned}
$$

The above equations are identical to those in reference [BK] except that the variation arguments are $k$ parameter or and $x$ parameter, instead of $\tau . F(\omega)$ is independent of $\tau$; thus $g(\omega)$ and $h(\omega)$ equations imply $F(\omega)=|P(i \omega)|^{2}-|Q(i \omega)|^{2}=0$ equation, but not the other way around. The system of $g(\omega)$, $h(\omega)$, and $F(\omega)=0$ can be solved numerically and graphically over the domain interest in the $(k, x)$ plane. First we solve the polynomial $F(\omega)=|P(i \omega)|^{2}-|Q(i \omega)|^{2}=0$, retaining only the real positive roots $\omega$, and discarding the others. The result is a $2 \mathrm{D}$ manifold (surface) $\omega=\omega(x, k)$ in a three

dimensional (3D) space $(x, k, \omega)$, where $\omega$ is continuous and differentiable with respect to its arguments, with the possible exception of infinite derivatives on 1D continuous line. We check which $\omega$ 's on the surface satisfy equations $g(\omega)=\ldots$, $h(\omega)=\ldots$ for some fixed value of $\tau$. The projection of these lines on the $(k, x)$ plane gives the loci of possible stability transitions of the Chemostat system. In the following, the subscripts $\lambda, \omega, \psi, \varphi, \gamma, \beta, k, \ldots$ indicate the corresponding partial derivatives. Let us first concentrate on $\Lambda(x)$, remember in $\lambda(\psi, \varphi, \gamma, \beta, k, \ldots)$ and $\omega(\psi, \varphi, \gamma, \beta, k, \ldots)$, and keeping all parameters except one $(x)$ and $\tau$. The derivation closely follows that in reference [BK]. Differentiating active circulator characteristic equation $P(\lambda)+Q(\lambda) e^{-\lambda \tau}=0$ with respect to specific parameter $(x)$, and inverting the derivative, for convenience, one calculates:

Remark: $x=\psi, \varphi, \gamma, \beta, k, \ldots$, etc

$$
\begin{aligned}
& \left(\frac{\partial \lambda}{\partial x}\right)^{-1} \\
& =\frac{-P_{\lambda}(\lambda, x) Q(\lambda, x)+Q_{\lambda}(\lambda, x) P(\lambda, x)-\tau P(\lambda, x) Q(\lambda, x)}{P_{x}(\lambda, x) Q(\lambda, x)-Q_{x}(\lambda, x) P(\lambda, x)}
\end{aligned}
$$

Where $P_{\lambda}=\frac{\partial P}{\partial \lambda}, \ldots$ etc., Substituting $\lambda=i \omega$, and bearing $\mathrm{i}$ $\overline{P(-i \omega)}=P(i \omega), \overline{Q(-i \omega)}=Q(i \omega)$ then $i P_{\lambda}(i \omega)=P_{\omega}(i \omega)$ and $i Q_{\lambda}(i \omega)=Q_{\omega}(i \omega)$ and that on the surface $|P(i \omega)|^{2}=|Q(i \omega)|^{2}$, one obtains: 
$\left.\left(\frac{\partial \lambda}{\partial x}\right)^{-1}\right|_{\lambda=i \omega}$

$=\left(\frac{i P_{\omega}(i \omega, x) \overline{P(i \omega, x)}+i Q_{\lambda}(i \omega, x) \overline{Q(\lambda, x)}-\tau|P(i \omega, x)|^{2}}{P_{x}(i \omega, x) \overline{P(i \omega, x)}-Q_{x}(i \omega, x) \overline{Q(i \omega, x)}}\right)$

Upon separating into real and imaginary parts, with

$$
\begin{gathered}
P=P_{R}+i P_{I} ; Q=Q_{R}+i Q_{I} ; P_{\omega}=P_{R \omega}+i P_{I \omega} \\
Q_{\omega}=Q_{R \omega}+i Q_{I \omega} ; P_{x}=P_{R x}+i P_{I x} \\
Q_{x}=Q_{R x}+i Q_{I x}
\end{gathered}
$$
rate's balance parameters. The dynamical behaviour of
(337) chemostat system with control tool is inspected deeply and stability analysis is done.

\section{APPENDIX A}

(338) A. Appendix $A_{1}$ (Lemma 1.1)

Assume that $\omega(\tau)$ is a positive and real root of $F(\omega, \tau)=0$ and defined for $\tau \in I$, which is continuous and differentiable. Assume further that if $\lambda=i \omega ; \omega \in R$, then $P_{n}(i \omega, \tau)+Q_{n}(i \omega, \tau) \neq 0, \tau \in \mathrm{R}$ hold true. Then the functions $S_{n}(\tau), n \in N_{0}$, are continuous and differentiable on $I$.

$\tau$ etc,. Where for convenience, we have dropped the arguments $(i \omega, x)$, and where

$$
\begin{aligned}
& F_{\omega}=2\left[\left(P_{R \omega} P_{R}+P_{I \omega} P_{I}\right)-\left(Q_{R \omega} Q_{R}+Q_{I \omega} Q_{I}\right)\right] \\
& F_{x}=2\left[\left(P_{R x} P_{R}+P_{I x} P_{I}\right)-\left(Q_{R x} Q_{R}+Q_{I x} Q_{I}\right)\right] \\
& \omega_{x}=-F_{x} / F_{\omega}
\end{aligned}
$$

We define $U$ and $V$ :

$$
\begin{gathered}
U=\left(P_{R} P_{I \omega}-P_{I} P_{R \omega}\right)-\left(Q_{R} Q_{I \omega}-Q_{I} Q_{R \omega}\right) \\
V=\left(P_{R} P_{I x}-P_{I} P_{R x}\right)-\left(Q_{R} Q_{I x}-Q_{I} Q_{R x}\right) \\
F_{\omega} \frac{\partial \omega}{\partial x}+F_{x}=0 ; x \in I \Rightarrow \frac{\partial \omega}{\partial x}=-\frac{F_{x}}{F_{\omega}} \\
\Lambda^{-1}(x)=\left(\frac{\partial R e \lambda}{\partial x}\right)_{\lambda=i \omega} ; \frac{\partial \omega}{\partial x}=\omega_{x}=-\frac{F_{x}}{F_{\omega}} \\
\Lambda(x)=\frac{2 P^{2}}{F_{x}^{2}+4 V^{2}} F_{\omega}\left(\tau \omega_{x}+\frac{U \omega_{x}+V}{P^{2}}\right) \\
\operatorname{sign}\left\{\Lambda^{-1}(x)\right\}=\operatorname{sign}\left\{\left(\frac{\partial \operatorname{Re} \lambda}{\partial x}\right)_{\lambda=i \omega}\right\} \\
\operatorname{sign}\left\{\Lambda^{-1}(x)\right\} \\
=\operatorname{sign}\left\{F_{\omega}\right\} \operatorname{sign}\left\{\tau \frac{\partial \omega}{\partial x}+\omega+\frac{U \frac{\partial \omega}{\partial x}+V}{|P|^{2}}\right\}
\end{gathered}
$$

\section{Conclusion}

A gradostat is a piece of laboratory apparatus for experiments along a nutrient gradient. It is a concentration of chemostats in which the adjacent vessels are connected in both directions. In that article we investigate the dynamical of chemostat which has initial quantities of nutrient, phage and bacteria. A chemostat is a continuous culture device in which microorganisms grow, and submitted to a flow of nutrients. There is an interaction between nutrient, phage and bacteria and it describes by some parameters. Important parameters are decay rate of phage in the vessel $(\varphi)$, growth rate of bacteria in the vessel $(\psi)$, interaction rate between bacteria and phage in the vessel $(\gamma)$, average number of free phage released at lysing in the vessel $(\beta)$, washout rate $(D)$, and talent period $(\tau)$. The control parameter in our system is the washout rate (dilution rate $D$ ) which is dependent on vessel's microorganism's concentration. We define very

\section{B. Appendix $A_{2}$ (Theorem 1.2)}

Assume that $\omega(\tau)$ is a positive real root of $F(\omega, \tau)=0$ defined for $\tau \in I, I \subseteq R_{+0}$, and at some $\tau^{*} \in I$,

$S_{n}\left(\tau^{*}\right)=0$. For some $n \in N_{0}$ then a pair of simple conjugate pure imaginary roots $\lambda_{+}\left(\tau^{*}\right)=i \omega\left(\tau^{*}\right)$ and $\lambda_{-}\left(\tau^{*}\right)=-i \omega\left(\tau^{*}\right)$ of $D(\lambda, \tau)=0$ exist at $\tau=\tau^{*}$ which crosses the imaginary axis from left to right if $\delta\left(\tau^{*}\right)>0$ and cross the imaginary axis from right to left if $\delta\left(\tau^{*}\right)<0$ where

$$
\begin{aligned}
& \delta\left(\tau^{*}\right)=\operatorname{sign}\left\{\left.\frac{d \mathrm{Re} \lambda}{d \tau}\right|_{\lambda=i \omega\left(\tau^{*}\right)}\right\}= \\
& \operatorname{sign}\left\{F_{\omega}\left(\omega\left(\tau^{*}\right), \tau^{*}\right)\right\} \operatorname{sign}\left\{\left.\frac{d S_{n}(\tau)}{d \tau}\right|_{\tau=\tau^{*}}\right\}
\end{aligned}
$$

The theorem becomes

$$
\begin{aligned}
& \operatorname{sign}\left\{\left.\frac{d \mathrm{Re} \lambda}{d \tau}\right|_{\lambda=i \omega \pm}\right\} \\
& =\operatorname{sign}\left\{ \pm \Delta^{1 / 2}\right\} \operatorname{sign}\left\{\left.\frac{d S_{n}(\tau)}{d \tau}\right|_{\tau=\tau^{+}}\right\}
\end{aligned}
$$

\section{Appendix $A_{3}$ (Theorem 1.3)}

The characteristic equation has a pair of simple and conjugate pure imaginary roots $\lambda= \pm \omega\left(\tau^{*}\right), \omega\left(\tau^{*}\right)$ real at $\tau^{*} \in I$ if $S_{n}\left(\tau^{*}\right)=\tau^{*}-\tau_{n}\left(\tau^{*}\right)=0$ for some $n \in N_{0}$. If $\omega\left(\tau^{*}\right)=\omega_{+}\left(\tau^{*}\right)$, this pair of simple conjugate pure imaginary roots crosses the imaginary axis from left to right if $\delta_{+}\left(\tau^{*}\right)>0$ and crosses the imaginary axis from right to left if $\delta_{+}\left(\tau^{*}\right)<0$ where

$$
\begin{aligned}
& \delta_{+}\left(\tau^{*}\right)=\operatorname{sign}\left\{\left.\frac{d \operatorname{Re} \lambda}{d \tau}\right|_{\lambda=i \omega_{+}\left(\tau^{*}\right)}\right\} \\
& =\operatorname{sign}\left\{\left.\frac{d S_{n}(\tau)}{d \tau}\right|_{\tau=\tau^{*}}\right\}
\end{aligned}
$$

If $\omega\left(\tau^{*}\right)=\omega_{-}\left(\tau^{*}\right)$, this pair of simple conjugate pure imaginary roots cross the imaginary axis from left to right if $\delta_{-}\left(\tau^{*}\right)>0$ and crosses the imaginary axis from right to left If $\delta_{-}\left(\tau^{*}\right)<0$ where

$$
\begin{aligned}
& \delta_{-}\left(\tau^{*}\right)=\operatorname{sign}\left\{\left.\frac{d \operatorname{Re} \lambda}{d \tau}\right|_{\lambda=i \omega_{-}\left(\tau^{*}\right)}\right\} \\
& =-\operatorname{sign}\left\{\left.\frac{d S_{n}(\tau)}{d \tau}\right|_{\tau=\tau^{*}}\right\}
\end{aligned}
$$

If $\omega_{+}\left(\tau^{*}\right)=\omega_{-}\left(\tau^{*}\right)=\omega\left(\tau^{*}\right)$ then $\Delta\left(\tau^{*}\right)=0$ and $\operatorname{sign}\left\{\left.\frac{d \operatorname{Re} \lambda}{d \tau}\right|_{\lambda=i \omega\left(\tau^{*}\right)}\right\}=0$ and the same is true when $S_{n}\left(\tau^{*}\right)=0$. The following result can be useful in identifying values of $\tau$ where stability switches happened. 
D. Appendix $A_{4}$ (Theorem 1.4)

Assume that for all $\tau \in I, \omega(\tau)$ is defined as a solution of $F(\omega, \tau)=0$ then $\delta_{ \pm}(\tau)=\operatorname{sign}\left\{ \pm \Delta^{1 / 2}(\tau)\right\} \operatorname{sign} D_{ \pm}(\tau)$.

\section{REFERENCES}

[1] R.-W. Lovitt, J.-W.-T Wimpenny, "The gradostat: A bidirectiona compound chemostat and its applications in microbiologica research" Joumal of General Microbiology, vol. 127, pp. 261-268 1981.

[2] E. Beretta, Y. Kuang, "Geometric stability switch criteria in delay differential systems with delay dependent parameters," SIAM J. Math. Anal., vol. 33, no. 5, pp. 1144-1165 2002

[3] H.-L. Smith. P. Waltman. The theory of the chemostat dynamic of micnbial competition 2008 Cambridge university press.

[4] Y.-A. Kuznetsov, Elements of applied bifurcation theory by Applied Mathematical Sciences.

[5] J.-K. Hale, Dynamics and Bifurcations by Texts in Applied Mathematics, Vol. 3

6] S.H. Strogatz, Nonlinear Dynamics and Chaos by Westyiew press.

Y. Kuang, Delay Differential equations with applications in population dynamics by Academic Press.

[8] I. Farmakis, M. Moskowitz Fixed point Theorems and their applications 2013 by World Scientific.

[9] K. Jiaoxun, C. Yuhao Stability of numerical methods for Delay Differential Equation (DDE) 2005 by Elsevier Science press.

[10] W.-H Steeb, C. Yuhao The Nonlinear workbook 2015 by World Scientific 6th.

[11] W. Jager, J.-W.-H. So, B. Tang, and P. Waltman "Competition in the gradostat," Joumal of Mathematical Biology, vol. 25, pp. 23-42

[12] H.-L. Smith, and B. Tang "Competition in the gradostat: The role of the communication rate," Journal of Mathematical Biology, vol. 27, pp. 139-165 1989

3] H.-L. Smith, B. Tang and P. Waltman "Competition in an n-vessel gradostat." SIAM Journal on Applied Mathematics. vol. 5. pp $1451-14711991$

[14] H. Smith, P. Waltman "The gradostat: A model of competition along a nutrient gradient," Microbial Ecology, vol. 22, pp. 207-226 1991. 15] TW. James "Continuous culture of microorganisms," Annual Review of Microbiology, vol. 15, pp. 27-46 1961.

[16] S. Govender, E.-P. Jacobs, W.-D. Leukes, and V.-L. Pillay "A scalable membrane gradostat reactor for enzyme production using phanerochaete chrysosporium," Biotechnology Letters, vol. 25, pp 127-131 2003

[17] S.-B. Hsu, and P. Waltman "On a system reaction diffusion equations arising from competition in an unstirred chemostat," SIAM J. Appl. Math, vol. 53, No. 4, pp. 1026-1044 1993

[18] A. Gaki, A. Theodorou, D.-V. Vayenas, and S. Pavlou "Complex dynamics of microbial competition in the gradostat," Journal of Biotechnology, vol. 139 , pp. 38-46 2009

[19] J.-S. Weitz, H. Hartman, and S.-A. Lavin "Coevolutionary arms races between bacteria and bacteriophage," PNAS, vol. 102, No.27.

[20] D.-A. Ratkowsky, J. Olley, and T. Ross "Unifying temperature effects on the growth rate of bacteria and the stability of globular proteins," Journal of Theoretical Biology, vol. 233, pp. 351-362.

[21] D. Vaque, J.-M. Gasol, and C. Marrase "Grazing rates on bacteria: significance of methodology and ecological factors," Marine ecology Progress series, vol. 109, pp. 263-274 1994.

[22] K. Nygaad, K.-Y. Borsheim, and T.-F. Thingstad "Grazing rates on bacteria by marine heterotrophic microflagellates compared uptake of bacteria-sized monodisperse fluorescent latex beads," Marine ecology Progress series, vol. 44, pp. 159-165 1988.

[23] M.-K. Czarnecka, R.-J. Chrost "Protozoans prefer large and metabolically active bacteria," Polish Joumal of Environmental Studies, vol. 12, No. 3, pp. 325-334 2003.

[24] R.-K. Poole, M.-J. Bazin and C.-W. Keevil "Microbial growth dynamics," Society for General Microbiology Special Publications, vol. 28,1990

[25] O. Arino, D.-E. Axelrod and M. Kimmel "Microbial growth dynamics Mathematical population dynamics: Proceedings of the Second International Conference" Lecture Notes in Pure and Applied Mathematics, 1991

[26] W.-T. Wimpenny, CRC handbook of laboratory model systems for microbial ecosystems (Volume I), Hardcover (Oct 31,1988).
[27] H.-L. Smith and P. Waltman, The theory of the chemostat: Dynamics of microbial competition (Cambridge Studies in Mathematical Biology), Hardcover ( $\operatorname{Jan} 27,1995$ ).

[28] J. Hofbauer,and W.-H. So "Competition in the gradostat the global stability problem," Nonlinear analysis theory Methods and

1017-1031 1994.

9] L.-M. Proctor L-M Okubo, and J.-A. Fuhrman "Calibrating of phage induced mortality in marine bacteria," Microb. Biol., vol. 25 pp. 39-58 1993.

[30] E. Beretta, Y. Kuang "Modeling and analysis of a marine bacteriophage infection with latency period," Nonlinear. Anal. Real World Appli., vol. 2, pp. 35-74 2001.

[31] S.-A. Gourley Y. Kuang "A delay reaction-diffusion model of the spread of bacteriophage infection," SIAM J. Appl. Math, vol. 65, pp. $550-5662005$

[32] A. Campbell "A conditions for existence of bacteriophage," Evolution, vol. 15, pp. 153-165 1961.

[33] J. Guckenheimer "Nonlinear oscillations dynamical systems and bifurcation of vector fields," Applied Mathematical Sciences, vol. 42

[34] S. Wiggins, Introdiction to applied nonlinear dynamical systems and chaos (Text in Applied Mathematics), Hardcover

[35] B. Balakumar, T.-K. Nagy, and D.-E. Gilsinn Delay differential equation: Recent advances and new directions (Springer), Hardcover (March 5,2009)

[36] E.-A. Butcher, H. Ma, E. Bueler, V. Averina and Z. Szabo "Stability of linear time periodic delay differential equations via chebyshev polynomials," Int. J. Numer. Meth. Engng, vol. 59, pp. 895-922

[37] G. Stepan, T. Insperger, and R. Szalai 'Delay parametric excitation and the nonlinear dynamics of cutting process," Int. J. of Bifurcation and Chaos, vol. 15, No. 9, pp. 2783-2798 2005.

[38] N.-K. Garg, B.-P. Mann, N.H. Kim and M.-H. Kurdi "Stability of a time delayed system with parametric excitation," Joumal of dynamic systems Measurement and control, vol. 129, 2007. 